TRANSACTIONS OF THE

AMERICAN MATHEMATICAL SOCIETY

Volume 359, Number 7, July 2007, Pages 3369-3407

S 0002-9947(07)04258-4

Article electronically published on February 12, 2007

\title{
ANALYTIC CONTRACTIONS, NONTANGENTIAL LIMITS, AND THE INDEX OF INVARIANT SUBSPACES
}

\author{
ALEXANDRU ALEMAN, STEFAN RICHTER, AND CARL SUNDBERG
}

\begin{abstract}
Let $\mathcal{H}$ be a Hilbert space of analytic functions on the open unit disc $\mathbb{D}$ such that the operator $M_{\zeta}$ of multiplication with the identity function $\zeta$ defines a contraction operator. In terms of the reproducing kernel for $\mathcal{H}$ we will characterize the largest set $\Delta(\mathcal{H}) \subseteq \partial \mathbb{D}$ such that for each $f, g \in \mathcal{H}, g \neq 0$ the meromorphic function $f / g$ has nontangential limits a.e. on $\Delta(\mathcal{H})$. We will see that the question of whether or not $\Delta(\mathcal{H})$ has linear Lebesgue measure 0 is related to questions concerning the invariant subspace structure of $M_{\zeta}$.

We further associate with $\mathcal{H}$ a second set $\Sigma(\mathcal{H}) \subseteq \partial \mathbb{D}$, which is defined in terms of the norm on $\mathcal{H}$. For example, $\Sigma(\mathcal{H})$ has the property that $\left\|\zeta^{n} f\right\| \rightarrow 0$ for all $f \in \mathcal{H}$ if and only if $\Sigma(\mathcal{H})$ has linear Lebesgue measure 0 .

It turns out that $\Delta(\mathcal{H}) \subseteq \Sigma(\mathcal{H})$ a.e., by which we mean that $\Delta(\mathcal{H}) \backslash$ $\Sigma(\mathcal{H})$ has linear Lebesgue measure 0 . We will study conditions that imply that $\Delta(\mathcal{H})=\Sigma(\mathcal{H})$ a.e.. As one corollary to our results we will show that if $\operatorname{dim} \mathcal{H} / \zeta \mathcal{H}=1$ and if there is a $c>0$ such that for all $f \in \mathcal{H}$ and all $\lambda \in \mathbb{D}$ we have $\left\|\frac{\zeta-\lambda}{1-\bar{\lambda} \zeta} f\right\| \geq c\|f\|$, then $\Delta(\mathcal{H})=\Sigma(\mathcal{H})$ a.e. and the following four conditions are equivalent:

(1) $\left\|\zeta^{n} f\right\| \nrightarrow 0$ for some $f \in \mathcal{H}$,

(2) $\left\|\zeta^{n} f\right\| \nrightarrow 0$ for all $f \in \mathcal{H}, f \neq 0$,

(3) $\Delta(\mathcal{H})$ has nonzero Lebesgue measure,

(4) every nonzero invariant subspace $\mathcal{M}$ of $M_{\zeta}$ has index 1, i.e., satisfies $\operatorname{dim} \mathcal{M} / \zeta \mathcal{M}=1$
\end{abstract}

\section{INTRODUCTION}

Let $\mathbb{D}$ denote the open unit disc in the complex plane and let $\zeta$ denote the identity function on $\mathbb{D}, \zeta(z)=z, z \in \mathbb{D}$. We will consider Hilbert spaces of analytic functions on the open unit disc $\mathbb{D}$ such that the operator $M_{\zeta}$ of multiplication with the function $\zeta$ acts as a contraction operator on $\mathcal{H}$, and we shall investigate the nontangential boundary behavior of functions in $\mathcal{H}$ or certain subspaces of $\mathcal{H}$ and the ramifications of this for the invariant subspace structure of $M_{\zeta}$. This paper may be considered to be a companion article to ARS2, where the same line of investigation was pursued for certain Banach spaces of analytic functions on $\mathbb{D}$, spaces that we will call analytic $P^{t}(\mu)$-spaces. Here $1 \leq t<\infty$ and $\mu$ is

Received by the editors July 11, 2005.

2000 Mathematics Subject Classification. Primary 47B32, 46E22; Secondary 30H05, 46E20.

Key words and phrases. Hilbert space of analytic functions, contraction, nontangential limits, invariant subspaces, index.

Part of this work was done while the second author visited Lund University. He would like to thank the Mathematics Department for its hospitality. Furthermore, work of the first author was supported by the Royal Swedish Academy of Sciences and work of the second and third authors was supported by the U. S. National Science Foundation.

(C)2007 American Mathematical Society C) 2007 American Mathematical Society
Reverts to public domain 28 years from publication 
a measure which is supported in the closed unit disc and such that the closure $P^{t}(\mu)$ of the polynomials in $L^{t}(\mu)$ is irreducible and has $\mathbb{D}$ as the set of its bounded point evaluations. Whereas in ARS2 the work is based on a detailed analysis of the properties of the measure $\mu$, in this paper the emphasis is on the use of the reproducing kernel for $\mathcal{H}$ and the minimal coisometric extension of the operator $M_{\zeta}$.

In this article we shall always at least assume that $\mathcal{H}$ is a space of analytic functions on $\mathbb{D}$ and satisfies the following two conditions:

(1.1) for each $\lambda \in \mathbb{D}$ the evaluation functional $f \rightarrow f(\lambda)$ is continuous on $\mathcal{H}$,

(1.2) $\zeta f \in \mathcal{H}$ for each $f \in \mathcal{H}$, and $\|\zeta f\| \leq\|f\|$.

Property (1.1) implies that $\mathcal{H}$ is a reproducing kernel Hilbert space; i.e., for each $\lambda \in \mathbb{D}$ there is a $k_{\lambda} \in \mathcal{H}$ such that $f(\lambda)=\left\langle f, k_{\lambda}\right\rangle$ for each $f \in \mathcal{H} . \quad k_{\lambda}$ is the reproducing kernel for $\mathcal{H}$. It follows from Property (1.2) that $M_{\zeta} f=\zeta f$ defines a contraction operator on $\mathcal{H}$. Using von Neumann's inequality it then follows that every bounded analytic function $\varphi$ on $\mathbb{D}$ defines a multiplication operator $M_{\varphi}$ on $\mathcal{H}$ with norm equal to the $H^{\infty}$-norm of $\varphi$.

For many of our results we will also require the following two conditions:

(1.3) for every $\lambda \in \mathbb{D}$ there is a $c_{\lambda}>0$ such that $\left\|\frac{\zeta-\lambda}{1-\overline{\lambda \zeta}} f\right\| \geq c_{\lambda}\|f\|$ for all $f \in \mathcal{H}$,

(1.4) $\operatorname{dim} \mathcal{H} \ominus \zeta \mathcal{H}=\operatorname{dim} \mathcal{H} \cap(\zeta \mathcal{H})^{\perp}=1$.

If $\mathcal{H}$ satisfies all conditions (1.1)-(1.4), then it follows from (1.1) and (1.2) that for each $\lambda \in \mathbb{D}$ and $f \in \mathcal{H}$ the functions $\frac{\zeta-\lambda}{1-\overline{\lambda \zeta}} f$ and $\frac{f}{1-\bar{\lambda} \zeta}$ are also in $\mathcal{H}$. Hence (1.3) just means that $M_{\zeta-\lambda}$ is bounded below. Note that (1.4) implies among other things that $\mathcal{H}$ is nonzero.

If $E$ is a measurable subset of the unit circle $\partial \mathbb{D}$, then we say that $\mathcal{H}$ admits nontangential limits on $E$ if for every $f, g \in \mathcal{H}, g \neq 0$, the ratio $f / g$ has nontangential limits a.e. on $E$, where a.e. stands for almost everywhere with respect to one-dimensional Lebesgue measure on $\partial \mathbb{D}$. At this point it is convenient to recall Privalov's Theorem, which states that a nonconstant meromorphic function in the unit disc cannot have nontangential limit equal to 0 (or $\infty$ ) on a set of positive measure in $\partial \mathbb{D}$, Ga, p. 94]. It follows that for $\mathcal{H}$ to admit nontangential limits on $E$ it is sufficient that there is just one nonzero function $g_{0}$ such that for every $f \in \mathcal{H}$ the ratio $f / g_{0}$ has nontangential limits a.e. on $E$. Thus in particular if the space $\mathcal{H}$ contains the constants, then $\mathcal{H}$ admits nontangential limits on $E$ if and only if each function in $\mathcal{H}$ has nontangential limits a.e. on $E$. We note that our definition of when $\mathcal{H}$ admits nontangential limits remains invariant if the space is multiplied by a nonzero analytic function. From this and the results in [Ri] it follows that if $\left(M_{\zeta}, \mathcal{H}_{1}\right)$ and $\left(M_{\zeta}, \mathcal{H}_{2}\right)$ are unitarily equivalent and satisfy (1.1)-(1.4), then they admit nontangential limits on the same set.

For each $f \in \mathcal{H}$ and $\lambda \in \mathbb{D}$ we have

$$
|f(\lambda)|=\left(1-|\lambda|^{2}\right)\left|\frac{f}{1-\bar{\lambda} \zeta}(\lambda)\right| \leq\left(1-|\lambda|^{2}\right)|| \frac{f}{1-\bar{\lambda} \zeta}\|\| k_{\lambda} \| .
$$

Thus if $g \in \mathcal{H}, g \neq 0$, we obtain the fundamental inequality

$$
\left|\frac{f}{g}(\lambda)\right|^{2} \leq\left(\left(1-|\lambda|^{2}\right)\left\|\frac{f}{1-\bar{\lambda} \zeta}\right\|^{2}\right)\left(\left(1-|\lambda|^{2}\right) \frac{\left\|k_{\lambda}\right\|^{2}}{|g(\lambda)|^{2}}\right) .
$$

If we let $v_{f}(\lambda)=\left(1-|\lambda|^{2}\right)\left\|\frac{f}{1-\bar{\lambda} \zeta}\right\|^{2}$, then by considering a unitary dilation of $M_{\zeta}$ one sees that $v_{f}$ has a harmonic majorant. We will give further details in Section 
2 , where we will also show that there is an $L^{1}(\partial \mathbb{D})$-function $w_{f, f}$ such that $v_{f}(\lambda)$ converges to $w_{f, f}(z)$ nontangentially for a.e. $z \in \partial \mathbb{D}$. It thus follows from the above that $f / g$ is in the Hardy space $H^{2}(\Omega)$ for every subregion $\Omega \subseteq \mathbb{D}$, where the expression $\left(1-|\lambda|^{2}\right) \frac{\left\|k_{\lambda}\right\|^{2}}{\left.g(\lambda)\right|^{2}}$ is bounded. Recall that $H^{2}(\Omega)$ is defined to be the space of all analytic functions $h$ on $\Omega$ such that $|h|^{2}$ has a harmonic majorant on $\Omega$.

Let $k$ be a function defined on the unit disc with values on the extended positive reals. The nontangential limit superior $K(z)$ of $k$ at $z \in \partial \mathbb{D}$ is defined to be the supremum of $A \in[0, \infty]$ such that there is a sequence $\left\{\lambda_{n}\right\}_{n \in \mathbb{N}}$ of points in the open unit disc that converges to $z$ nontangentially and such that $\left\{k\left(\lambda_{n}\right)\right\}_{n \in \mathbb{N}} \rightarrow A$. We write $K(z)=\mathrm{nt}_{\lambda \rightarrow z} \varlimsup_{\substack{\text { am } \\ k}}(\lambda)$. A standard argument shows that if $k$ is continuous, then $K$ is measurable and for each angle $\alpha, K(z)$ equals for a.e. $z \in \partial \mathbb{D}$ the limit superior of $k(\lambda)$, where $z$ is approached from within a fixed Stolz angle with opening $\alpha$.

For a nonzero function $g \in \mathcal{H}$ we will consider the set

$$
\Delta_{g}(\mathcal{H})=\left\{z \in \partial \mathbb{D}: \operatorname{nt}_{\lambda \rightarrow z} \varlimsup_{i m}\left(1-|\lambda|^{2}\right) \frac{\|\left. k_{\lambda}\right|^{2}}{|g(\lambda)|^{2}}<\infty\right\} .
$$

Roughly speaking this is the boundary set where the normalized reproducing kernel $\frac{k_{\lambda}(w)}{\overline{g(\lambda)} g(w)}$ is nontangentially bounded by the Hardy kernel $(1-\bar{\lambda} w)^{-1}$. Of course the definition is motivated by the earlier discussion. Furthermore, the remark above implies that $\Delta_{g}(\mathcal{H})$ is always measurable, and we shall prove that the $\mathrm{nt}_{\lambda \rightarrow z} \varlimsup_{i m}\left(1-|\lambda|^{2}\right) \frac{\left.|| k_{\lambda}\right|^{2}}{|g(\lambda)|^{2}}$ exists and is finite for a.e. $z \in \Delta_{g}(\mathcal{H})$ (see Proposition 3.5). In Section 3 we shall see that it follows from standard results and the discussion above that $\mathcal{H}$ admits nontangential limits on $\Delta_{g}(\mathcal{H})$. We will also see that up to a set of measure 0 the set $\Delta_{g}(\mathcal{H})$ is independent of the choice of the nonzero function $g$. We shall thus drop the subscript $g$ and write $\Delta(\mathcal{H})$ for the set of all Lebesgue points of $\Delta_{g}(\mathcal{H})$. Thus, $\mathcal{H}$ admits nontangential limits on $\Delta(\mathcal{H})$, and in Section 4 we will show that up to sets of measure zero $\Delta(\mathcal{H})$ is the largest set with this property.

Theorem 1.1. Suppose $\mathcal{H}$ satisfies (1.1) and (1.2). Then

(a) for every $f, g \in \mathcal{H}, g \neq 0$ the function $f / g$ has nontangential limits a.e. on $\Delta(\mathcal{H})$

(b) for every $g \in \mathcal{H}, g \neq 0$, there is a function $f \in \mathcal{H}$ such that for a.e. $z \in$ $\partial \mathbb{D} \backslash \Delta(\mathcal{H})$ there is a sequence $\left\{\lambda_{n}\right\}$ in $\mathbb{D}$ that converges to $z$ nontangentially and such that $\left|\frac{f\left(\lambda_{n}\right)}{g\left(\lambda_{n}\right)}\right| \rightarrow \infty$ as $n \rightarrow \infty$.

Theorem 1.1 (a) will be proved as Corollary 3.4 and Theorem 1.1 (b) will follow from Theorem 4.1. In fact as one might guess from the discussion above, we shall see that $f / g \in H^{2}(\Omega)$ for some appropriate nontangential approach region $\Omega \subseteq \mathbb{D}$. Also, if we assume in addition that the polynomials are dense in $\mathcal{H}$, then we shall see that there is a nonzero function $f \in \mathcal{H}$ such that the union of $\Delta(\mathcal{H})$ and the nontangential limit set of the zeros of $f$ has full measure in $\partial \mathbb{D}$ (see Theorem 4.1 (b)).

Theorem 1.1 gives a useful description of the set where $\mathcal{H}$ admits nontangential limits if one has asymptotic knowledge of $\left\|k_{\lambda}\right\|$ as $\lambda$ approaches $\partial \mathbb{D}$ nontangentially. For many applications it would be helpful if one had a more direct way to determine $\Delta(\mathcal{H})$, say from the norm in the space. 
In the following we shall write $|E|$ for the Lebesgue measure of a measurable set $E \subseteq \partial \mathbb{D}$, and we shall say $E \subseteq F$ a.e. if $|E \backslash F|=0$.

Let $\mu$ be a nonnegative finite Borel measure with support in the closure of $\mathbb{D}$. By $P^{2}(\mu)$ we mean the closure of the polynomials in $L^{2}(\mu)$. One can identify the space $P^{2}(\mu)$ with a Hilbert space of analytic functions satisfying conditions (1.1) and (1.2) if and only if $P^{2}(\mu)$ is irreducible (i.e., $P^{2}(\mu)$ contains no nontrivial characteristic functions) and the open unit disc equals the set of analytic bounded point evaluations of $P^{2}(\mu)$. In fact by (1.1) it is clear that one needs the point evaluations to be bounded, and the irreducibility is needed to assure that 0 is the only function in the space that is identically equal to zero in the open unit disc. We shall call such a space an analytic $P^{2}(\mu)$-space. It is well known and easy to see that if $\mathcal{H}$ is an analytic $P^{2}(\mu)$-space with measure $\mu$, then $d \mu \mid \partial \mathbb{D}=h \frac{|d z|}{2 \pi}$ for some nonnegative $L^{1}(\partial \mathbb{D})$-function $h$; see e.g. Proposition 4.1 of ARR.

It is one of the main results of ARS2 that in this case

$$
\Delta(\mathcal{H})=\{z \in \partial \mathbb{D}: h(z)>0\} \text { a.e. }
$$

More precisely, it was shown that under this hypothesis

$$
\mathrm{nt}_{\lambda \rightarrow z} \varlimsup_{\frac{1}{\left(1-|\lambda|^{2}\right)|| k_{\lambda}||^{2}}}=h(z) \text { for a.e. } z \in \partial \mathbb{D},
$$

and that for every $f \in P^{2}(\mu)$ the nontangential limit function of the analytic extension of $f$ to $\mathbb{D}$ agrees a.e. on $\Delta(\mathcal{H})$ with the values of $f$ as an element in $L^{2}(\mu \mid \partial \mathbb{D})$.

In particular it follows that $\Delta\left(P^{2}(\mu)\right)$ has measure zero if and only if $\left\|z^{n}\right\|_{P^{2}(\mu)} \rightarrow$ 0 as $n \rightarrow \infty$.

It is easy to construct examples of non- $P^{2}(\mu)$-spaces $\mathcal{H}$ satisfying (1.1) and (1.2) and such that $\Delta(\mathcal{H})$ has measure zero, but $1 \in \mathcal{H}$ and $\left\|z^{n}\right\| \nrightarrow 0$. For example, let $g$ be an analytic function in $\mathbb{D}$ that has nontangential limits almost nowhere on $\partial \mathbb{D}$, and set $\mathcal{H}=\left\{f_{1}+g f_{2}: f_{1}, f_{2} \in H^{2}(\mathbb{D})\right\},\left\|f_{1}+g f_{2}\right\|^{2}=\left\|f_{1}\right\|_{H^{2}}^{2}+\left\|f_{2}\right\|_{H^{2}}^{2}$. Then $M_{\zeta}$ is isometric and $k_{\lambda}(z)=(1+\overline{g(\lambda)} g(z)) /(1-\bar{\lambda} z)$, and it follows that $\Delta(\mathcal{H})$ has measure 0 . Thus looking at the case $\mathcal{H}=P^{2}(\mu)$ it seems natural to add the conditions (1.3) and (1.4). We note that analytic $P^{2}(\mu)$-spaces satisfy these conditions.

If one is given a norm for a space $\mathcal{H}$ that is not necessarily a $P^{2}(\mu)$-space, then where might one expect the functions in $\mathcal{H}$ to have some kind of regular nontangential boundary behaviour? That is, is there a way to determine $\Delta(\mathcal{H})$ that does not rely on estimating $\left\|k_{\lambda}\right\|$ at least asymptotically for $\lambda$ near $\partial \mathbb{D}$ ? Our construction is guided by the $P^{2}(\mu)$-situation.

Construction of the set $\Sigma(\mathcal{H})$ :

For $f \in \mathcal{H}$ we set $\|f\|_{*}=\lim _{n \rightarrow \infty}\left\|\zeta^{n} f\right\|$ and $\mathcal{A}=\left\{f \in \mathcal{H}:\|f\|_{*}=0\right\}^{\perp}$ so that $\|\cdot\|_{*}$ defines an inner product norm on $\mathcal{A}$, and clearly $\|\zeta f\|_{*}=\|f\|_{*}$ for every $f \in \mathcal{H}$. We let $P_{\mathcal{A}}$ denote the projection of $\mathcal{H}$ onto $\mathcal{A}$ and we set $S f=P_{\mathcal{A}} M_{\zeta} f, f \in \mathcal{A}$. Then $\|S f\|_{*}=\|f\|_{*}$ for every $f \in \mathcal{A}$, so $S$ extends to be an isometric operator on the completion of $\mathcal{A}$ with respect to $\|\cdot\|_{*}$ (see Section 2 for more details). This isometric operator has a minimal unitary extension $U$, which acts on some Hilbert space $\mathcal{K}$. We shall see that the spectral measure of $U$ is absolutely continuous (Lemma 2.1). We note that one can show that this operator $U$ is unitarily equivalent to the unitary summand of the minimal coisometric extension of the contraction $M_{\zeta}$ (the so-called ${ }^{*}$-residual part of the minimal unitary dilation of $\left.M_{\zeta}, \overline{\mathrm{NF}}\right)$. Let $\Sigma$ be a 
measurable set such that $\chi_{\Sigma}(z)|d z|$ is a scalar-valued spectral measure for $U$ (see Co, p.286). This set is unique only up to sets of measure zero, but if we let $\Sigma(\mathcal{H})$ denote the set of Lebesgue points of $\Sigma$, then we get a set uniquely associated with the Hilbert space $\mathcal{H}$.

We note that for arbitrary absolutely continuous contractions this set has played a role in the theory of dual algebras. In this context it often has been denoted by $\Sigma_{*}$; see e.g. $\mathrm{CEP}$.

It is clear that if $\mathcal{H}$ is an analytic $P^{2}(\mu)$-space, then the operator $U$ is just multiplication with the identity function acting on $L^{2}(\mu \mid \partial \mathbb{D})$. Hence it follows from equation (1.6) that $\Delta\left(P^{2}(\mu)\right)=\Sigma\left(P^{2}(\mu)\right)$ a.e. for every analytic $P^{2}(\mu)$-space.

We shall see that for every polynomial $p$ and every $f \in \mathcal{H}$ one has $\|p f\|_{*}^{2}=$ $\int_{\partial \mathbb{D}}|p(z)|^{2} w_{f, f}(z) \frac{|d z|}{2 \pi}$. Recall that we introduced the function $w_{f, f}$ in the paragraph following the fundamental inequality (1.5). Thus, for each $f \in \mathcal{H}$ we have

$$
\Delta(\mathcal{H}) \subseteq\left\{z \in \partial \mathbb{D}: w_{f, f}(z)>0\right\} \subseteq \Sigma(\mathcal{H}) \text { a.e. }
$$

Here the first inclusion follows by setting $f=g$ in (1.5) and taking nontangential limits; the second inclusion will follow from Lemma 2.2 below.

Note that $|\Sigma(\mathcal{H})|=0$ if and only if $\left\|\zeta^{n} f\right\| \rightarrow 0$ for every $f \in \mathcal{H}$. Thus, an answer to the following question might yield information that does not readily follow from Theorem 1.1.

Question 1.2. If the space $\mathcal{H}$ satisfies the conditions (1.1) and (1.2), then under what additional conditions does

$$
\Delta(\mathcal{H})=\Sigma(\mathcal{H}) \text { a.e.? }
$$

If we once more take $f=g$ in the fundamental inequality (1.5) and then take a nontangential lim-inf, then we obtain for all $f \in \mathcal{H}$ that

$$
1 \leq w_{f, f}(z) \underset{\lambda \rightarrow-\varliminf_{\rightarrow z}}{ }\left(1-|\lambda|^{2}\right) \frac{\left\|k_{\lambda}\right\|^{2}}{|f(\lambda)|^{2}} \text { for a.e. } z \in \partial \mathbb{D} \text {. }
$$

This implies by Lemma 2.2 below that in the a.e. sense

$$
\left\{z \in \partial \mathbb{D}: \operatorname{nt}_{\lambda \rightarrow z} \frac{\left(1-|\lambda|^{2}\right)|| k_{\lambda}||^{2}}{|f(\lambda)|^{2}} \neq \underset{\lambda t-\varliminf_{\rightarrow z}}{ } \frac{\left.\left(1-|\lambda|^{2}\right)|| k_{\lambda}\right|^{2}}{|f(\lambda)|^{2}}\right\} \subseteq \Sigma(\mathcal{H}) \backslash \Delta(\mathcal{H}) .
$$

See Proposition 3.5 for the fact that the nontangential limit exists a.e. on $\Delta(\mathcal{H})$. This observation can be used to construct further examples which show that some extra conditions are needed to obtain a positive answer to Question 1.2. See Sections 7 and 8 for further details.

We already mentioned that one might add conditions (1.3) and (1.4), but Example 8.1 will show that there are spaces $\mathcal{H}$ that satisfy conditions (1.1)-(1.4) but with $|\Delta(\mathcal{H})|=0$ and $\Sigma(\mathcal{H})=\partial \mathbb{D}$.

We will give two types of conditions that will imply the a.e. equality of the sets in Question 1.2. In Section 7 we will develop a technique that for example can be used to show that if the reproducing kernel $k$ of $\mathcal{H}$ is of the form $k_{\lambda}(z)=\frac{1}{1-\bar{\lambda} z} \frac{1}{1-u_{\lambda}(z)}$ for some positive definite and sesquianalytic function $u_{\lambda}(z)$ with $u_{\lambda}(\lambda)<1$ for all $\lambda \in \mathbb{D}$, then

$$
\Delta(\mathcal{H})=\Sigma(\mathcal{H})=\left\{z \in \partial \mathbb{D}: \operatorname{nt}_{\lambda \rightarrow z} \lim _{\lambda}(\lambda)<1\right\} \text { a.e. }
$$


In fact we shall focus on the related case where $k_{\lambda}(z)$ is of the form

$$
(1-\bar{\lambda} z) k_{\lambda}(z)=\sum_{n \geq 0} \overline{\varphi_{n}(\lambda)} \varphi_{n}(z)
$$

for some analytic functions $\varphi_{n}$ with $\varphi_{n} / \varphi_{0}$ in the Nevanlinna class of $\mathbb{D}$. In Theorem 7.4 we will show that in the a.e. sense

$$
\Sigma(\mathcal{H}) \backslash \Delta(\mathcal{H}) \subseteq\left\{z \in \partial \mathbb{D}: \sum_{n \geq 0}\left|\frac{\varphi_{n}}{\varphi_{0}}(z)\right|^{2} \neq \operatorname{nt}_{\lambda \rightarrow z} \lim _{n}\left(1-|\lambda|^{2}\right) \frac{k_{\lambda}(\lambda)}{\left|\varphi_{0}(\lambda)\right|^{2}}\right\} .
$$

The other type of hypothesis that we shall impose on $\mathcal{H}$ for an answer to Question 1.2 will be discussed in Section 5. In particular, Theorem 5.2 will imply that $\Delta(\mathcal{H})=\Sigma(\mathcal{H})$ if we have the following uniform version of condition (1.3):

(1.8) there is a $c>0$ such that $\left\|\frac{\zeta-\lambda}{1-\bar{\lambda} \zeta} f\right\| \geq c\|f\|$ for all $f \in \mathcal{H}$ and all $\lambda \in \mathbb{D}$.

Condition (1.8) is often easy to check. If $\|\cdot\|_{\mathcal{K}}$ is a norm on the polynomials such that condition (1.8) is satisfied for every polynomial $f$, then the norm $\|f\|^{2}=$ $\|f\|_{\mathcal{K}}^{2}+\int_{\partial \mathbb{D}}|f|^{2} h|d z|$ will satisfy (1.8) for every nonnegative $L^{1}(\partial \mathbb{D})$-function $h$. Thus if $M_{\zeta}$ denotes the operator of multiplication by $\zeta$ on the closure $\mathcal{H}$ of the polynomials in this norm, if $M_{\zeta}$ is a contraction and irreducible, and if for each $\lambda \in \mathbb{D}$ there is a $c_{\lambda}>0$ such that $|f(\lambda)| \leq c_{\lambda}\|f\|$ for every polynomial $f$, then $\mathcal{H}$ can be viewed as a space of analytic functions on $\mathbb{D}$ and it will satisfy (1.1)-(1.4) and (1.8). For example, it is well known that this is the case if $\mathcal{K}$ is a weighted Bergman space with standard weight, i.e., if $\mathcal{K}=P^{2}\left(\left(1-|z|^{2}\right)^{\alpha} d A(z)\right), \alpha>-1$, $A=$ area measure on $\mathbb{D}$ (see [HKZ or ARS3, Section 4 ). In this case the resulting space $\mathcal{H}$ will, of course, be an analytic $P^{2}(\mu)$-space. In [ARS3, Corollary 4.3, it was shown that this hypothesis is also satisfied for certain other spaces $\mathcal{K}$ where $\left(M_{\zeta}, \mathcal{K}\right)$ is a weighted shift operator. We also mention that it follows from the proof of Proposition 4.10 of $\mathrm{MR}$ ] that all Hilbert spaces with "Bergman-type kernels" satisfy condition 1.8 (see $\mathrm{MR}$ for definitions).

Nevertheless there are spaces $\mathcal{H}$ satisfying (1.1)-(1.4), where condition (1.8) fails. Thus it will be useful to know that the uniform inequality (1.8) is only required for $\lambda$ in an appropriate subset of $\mathbb{D}$. In order to explain this we define the quantity

$$
c_{\mathcal{H}}(\lambda)=\inf \left\{\left\|\frac{\zeta-\lambda}{1-\bar{\lambda} \zeta} f\right\|:\|f\|=1\right\},
$$

and we note that $0<c_{\mathcal{H}}(\lambda) \leq 1$ for all $\lambda \in \mathbb{D}$. We set

$$
\Gamma(\mathcal{H})=\left\{z \in \partial \mathbb{D}: \underset{\lambda \rightarrow z}{\operatorname{nt}} \lim _{\mathcal{H}}(\lambda)>0\right\} .
$$

For analytic $P^{2}(\mu)$-spaces one can show that $\Delta(\mathcal{H}) \subseteq \Gamma(\mathcal{H})$ a.e. (see Section 9), and in general it is clear that, if condition (1.8) is satisfied, then $\Gamma(\mathcal{H})$ has full measure in $\partial \mathbb{D}$.

The following theorem implies an answer to Question 1.2 on $\Gamma(\mathcal{H})$, and it provides an analogue of (1.7). In fact, we consider it a computable asymptotic estimate on the size of $\left\|k_{\lambda}\right\|$.

Theorem 1.3. If $\mathcal{H}$ satisfies (1.1)-(1.4), then

$$
\Delta(\mathcal{H}) \cap \Gamma(\mathcal{H})=\Sigma(\mathcal{H}) \cap \Gamma(\mathcal{H}) \text { a.e. }
$$


and for every $g \in \mathcal{H}$ we have

$$
w_{g, g}(z)=\mathrm{nt}_{\lambda \rightarrow z} \lim _{\lambda \rightarrow \mid} \frac{|g(\lambda)|^{2}}{\left(1-|\lambda|^{2}\right)|| k_{\lambda} \|^{2}} \quad \text { for a.e. } z \in \Gamma(\mathcal{H}) \cup(\partial \mathbb{D} \backslash \Sigma(\mathcal{H})) .
$$

Furthermore, for every $f, g \in \mathcal{H}, g \neq 0$ we have $w_{f, f}=\left|\frac{f}{g}\right|^{2} w_{g, g}$ a.e. on $\Delta(\mathcal{H}) \cap \Gamma(\mathcal{H})$ and if $\Sigma(\mathcal{H}) \subseteq \Gamma(\mathcal{H})$ a.e., then

$$
\|f\|_{*}^{2}=\left\|w_{f, f}\right\|_{L^{1}}=\int_{\Delta(\mathcal{H})}\left|\frac{f}{g}(z)\right|^{2} w_{g, g}(z) \frac{|d z|}{2 \pi} .
$$

Here $\frac{f}{g}(z)$ denotes the nontangential limit of the meromorphic function $\frac{f}{g}$, which by Theorem 1.1 exists a.e. on $\Delta(\mathcal{H})$.

Of course, outside of $\Sigma(\mathcal{H})$ each $w_{g, g}=0$. Thus, if $\Sigma(\mathcal{H}) \subseteq \Gamma(\mathcal{H})$ a.e., then the definition $\Delta(\mathcal{H})$ together with the remarks made earlier implies that $\Delta(\mathcal{H})=$ $\Delta_{g}(\mathcal{H})=\left\{z \in \partial \mathbb{D}: w_{g, g}(z)>0\right\}$, and this is independent of $g \neq 0$. Thus, if $|\Delta(\mathcal{H})|>0$, then $\|g\|_{*}^{2}=\left\|w_{g, g}\right\|_{L^{1}} \neq 0$ for all $g \in \mathcal{H}, g \neq 0$. Hence the following corollary is a simple consequence:

Corollary 1.4. If $\Sigma(\mathcal{H}) \subseteq \Gamma(\mathcal{H})$ a.e., then the following dichotomy holds: either $\left\|z^{n} f\right\| \rightarrow 0$ for all $f \in \mathcal{H}$ or $\left\|z^{n} f\right\| \rightarrow 0$ only for $f=0$.

Suppose now that $\mathcal{H}$ satisfies (1.1)-(1.4) and let $k$ be its reproducing kernel. We shall write Lat $\mathcal{M}_{\zeta}$ for the collection of closed subspaces of $\mathcal{H}$ that are invariant for $M_{\zeta}$. If $\mathcal{M} \in \operatorname{Lat} M_{\zeta}$, then $\mathcal{M}$ automatically satisfies conditions (1.1)-(1.3). The dimension of $\mathcal{M} \ominus \zeta \mathcal{M}$ is called the index of the invariant subspace. Thus the index is either a nonnegative integer or $\infty$, and it has turned out to be an important object to consider when investigating the structure of Lat $M_{\zeta}$. It is easy to check that only (0) has index 0 , and by (1.4) $\mathcal{H}$ has index 1 . Invariant subspaces of index 1 always exist in abundance. If $f$ is any nonzero function in $\mathcal{H}$, and if $[f]$ denotes the closure of the polynomial multiples of $f$, then $[f]$ has index 1. Similarly, any nontrivial zero-based invariant subspace has index 1 . These last two assertions are relatively easy to prove; see e.g. Ri], where also a number of other elementary properties of the index function are presented.

If $\mathcal{H}=H^{2}(\mathbb{D})$, then by Beurling's theorem all nonzero invariant subspaces are of the type $[f]$, so in this case they all have index 1 . On the other hand, it is known that there are spaces $\mathcal{H}$ that contain invariant subspaces of arbitrary index.

Index $n$ invariant subspaces can be represented as spaces of vector-valued analytic functions. In fact if $\mathcal{H}=L_{a}^{2}$, the Bergman space of square area integrable functions on $\mathbb{D}$, and if $\mathcal{M} \in \operatorname{Lat}\left(M_{\zeta}, L_{a}^{2}\right)$, then the restricted operator $M_{\zeta} \mid \mathcal{M}$ is unitarily equivalent to a multiplication operator $M_{\zeta}$ modelled on a space of vectorvalued analytic functions on $\mathbb{D}$, where the values lie in a Hilbert space of dimension equal to ind $\mathcal{M}$, $\mathrm{MR}$.

The results of ABFP imply that invariant subspaces of index $>1$ occur whenever $\left\|\zeta^{n} f\right\| \rightarrow 0$ for each $f \in \mathcal{H}$ as $n \rightarrow \infty$. This is equivalent to saying that invariant subspaces of index $>1$ exist whenever $|\Sigma(\mathcal{H})|=0$. In the absence of a more general answer to Question 1.2 we have the following improvement of this result and a partial converse.

Theorem 1.5. If $\mathcal{H}$ satisfies (1.1)-(1.4), then

(a) if $|\Delta(\mathcal{H})|=0$, then there is an $\mathcal{M} \in \operatorname{Lat} M_{\zeta}$ with ind $\mathcal{M}>1$,

(b) if $|\Delta(\mathcal{H}) \cap \Gamma(\mathcal{H})|>0$, then every nonzero $\mathcal{M} \in \operatorname{Lat} M_{\zeta}$ has index 1. 
For the proof of (a) we use some of the ideas and results of ARS1 together with an explicit construction of a sequence that is dominating for the unit circle as well as interpolating for an appropriate subspace of $\mathcal{H}$. As a corollary we obtain:

Corollary 1.6. If $\mathcal{H}$ satisfies (1.1)-(1.4) and if $\Sigma(\mathcal{H}) \subseteq \Gamma(\mathcal{H})$ a.e., then the following conditions are equivalent:

(a) every nonzero $\mathcal{M} \in \operatorname{Lat} M$ has index 1 ,

(b) $\mathcal{H}$ admits nontangential limits on a set of positive measure (i.e. $|\Delta(\mathcal{H})|>0$ ),

(c) $\left\|\zeta^{n} f\right\| \nrightarrow 0$ for all $f \in \mathcal{H}, f \neq 0$,

(d) $\left\|\zeta^{n} f\right\| \nrightarrow 0$ for some $f \in \mathcal{H}$.

By Theorem 1.3 the hypotheses imply that $\Delta(\mathcal{H})=\Sigma(\mathcal{H})=\Delta(\mathcal{H}) \cap \Gamma(\mathcal{H})$ a.e.; thus the corollary follows immediately from Corollary 1.4 and Theorem 1.5. In ARS2 it was shown that the conclusion of this corollary holds for all analytic $P^{2}(\mu)$-spaces.

If the hypothesis that $\Sigma(\mathcal{H}) \subseteq \Gamma(\mathcal{H})$ a.e. is dropped from the statement and we only assume that $\mathcal{H}$ satisfies (1.1)-(1.4), then Theorem 1.5 shows that $(a) \Longrightarrow(b)$, $(b) \Longrightarrow(c)$ can be deduced from the theorem of Khinchin and Kolmogorov Du, p. 226], and, of course, $(c) \Longrightarrow(d)$ is trivial. Our examples in Section 8 will show that neither (b) and (c) nor (c) and (d) are equivalent. We do not know whether (a) and (b) are equivalent (see also Question 9.3).

In spaces $\mathcal{H}$ that are known to contain invariant subspaces with high index it becomes important to determine which functions $f \in \mathcal{H}$ are contained in such invariant subspaces and which are not. We note that it does not appear to be of interest to distinguish further cases dependent on what the high index exactly equals, because in Section 9 we shall show the following result: if $\left\|\zeta^{n} g\right\| \rightarrow 0$ for every $g \in \mathcal{H}$, and if $f \in \mathcal{H}$ is contained in an invariant subspace of index $>1$, then for any $n \geq 1$ or $n=\infty, f$ is contained in an invariant subspace of index $n$. In fact, that will be an easy consequence of the results of [ARS1] and ABFP; see Proposition 9.4.

On the other hand there will be many invariant subspaces that have the property that all larger invariant subspaces have index 1 . For example, it is a very simple result that if the polynomials are dense in $\mathcal{H}$ and if $f$ is a bounded function, then every invariant subspace containing $f$ must have index 1; see [Ri], Proposition 3.6. For analytic $P^{2}(\mu)$-spaces more results of this type are contained in $\mathrm{AR}$. For weighted Bergman spaces also see [Ya, WY] and ARS1. In [ARS1 the majorization function of an invariant subspace $\mathcal{M}$ was introduced in order to study this problem,

$$
k_{\mathcal{M}}(\lambda)=\frac{\sup \{|f(\lambda)|: f \in \mathcal{M},\|f\| \leq 1\}}{\sup \{|f(\lambda)|: f \in \mathcal{H},\|f\| \leq 1\}} .
$$

Since $\mathcal{H}$ is a Hilbert space, it is clear that $k_{\mathcal{M}}(\lambda)=\frac{\left\|P_{\mathcal{M}} k_{\lambda}\right\|}{\left\|k_{\lambda}\right\|}$, where $P_{\mathcal{M}}$ denotes the projection of $\mathcal{H}$ onto $\mathcal{M}$. It is easy to see that for the Hardy space $H^{2}(\mathbb{D})$ one has $k_{\mathcal{M}}(\lambda)=|\varphi(\lambda)|$ whenever $\varphi$ is a classical inner function with $\mathcal{M}=\varphi H^{2}(\mathbb{D})$. We note that this implies that the majorization function of any nonzero invariant subspace of $H^{2}(\mathbb{D})$ has nontangential limit equal to one. For the Bergman space $L_{a}^{2}$ it was shown in ARS1 that if $\mathcal{M}$ is an invariant subspace of index 1 , then for a.e. $z \in \partial \mathbb{D}$ either $k_{\mathcal{M}}(\lambda) \rightarrow 1$ as $\lambda \rightarrow z$ nontangentially or the nontangential lim-inf of $k_{\mathcal{M}}(\lambda)$ is zero at $z$. In general, determining the relationship between the boundary 
behaviour of a given function $f \in \mathcal{H}$ and the nontangential boundary behaviour of the majorization function $k_{[f]}(\lambda)$ is a very delicate task. We refer the reader to [ARS1, Section 5] for some results for the weighted Bergman spaces. We will see in Section 6 that for every nonzero invariant subspace $\mathcal{M}, \underset{\lambda \rightarrow z}{\operatorname{nt}} \lim _{\mathcal{M}} k_{\mathcal{M}}(\lambda)=1$ a.e. on $\Sigma(\mathcal{H}) \cap \Gamma(\mathcal{H})$. Thus once one knows Corollary 1.6 the following theorem is of interest only if $\mathcal{H}$ does not admit any nontangential limits on any set of positive measure.

Theorem 1.7. Suppose $\mathcal{H}$ is a Hilbert space of analytic functions satisfying (1.1)(1.4) and that $\Gamma(\mathcal{H})$ has full measure in $\partial \mathbb{D}$. Let $\mathcal{M} \in$ Lat $M_{\zeta}$ with ind $\mathcal{M}=1$.

Then $\mathcal{M}$ is contained in an invariant subspace of index $>1$ if and only if for a.e. $z \in \partial \mathbb{D}$ the nontangential lim-inf of $k_{\mathcal{M}}(\lambda)$ at $z$ is 0 .

This will follow from Theorem 6.4. For the weighted Bergman spaces $\mathcal{H}=$ $P^{2}\left(w_{\alpha} d A\right), w_{\alpha}(z)=\left(1-|z|^{2}\right)^{\alpha}, \alpha>-1$ this theorem was proved in ARS1. Furthermore the sufficiency of the nontangential lim-inf condition on the majorization function for the existence of high index invariant subspaces is true for all Hilbert spaces satisfying conditions (1.1)-(1.4), and this was also shown in ARS1. Our new contribution here is the proof of the necessity under the hypothesis that $\Gamma(\mathcal{H})$ has full measure (or under hypothesis (1.8)). We note that this adds new information even in the context of the weighted Bergman spaces, because Theorem 1.7 automatically yields information about the existence of high index invariant subspaces between two given index 1 invariant subspaces; see the discussion after Theorem 6.4. We also mention that in Corollary 4.3 of ARS3 it was shown that a certain class of weighted shift operators $M_{\zeta}$ satisfies the hypotheses of Theorem 1.7.

For two individual functions we obtain the following result:

Theorem 1.8. Suppose $\mathcal{H}$ is a Hilbert space of analytic functions satisfying (1.1)(1.4).

If $f, g \in \mathcal{H}, g \neq 0$ such that $f / g$ has a nontangential limit on $E \subseteq \Gamma(\mathcal{H})$ with $|E|>0$, then ind $[f, g]=1$.

The special case of $\mathcal{H}=L_{a}^{2}$ of this theorem was known, ARR, Corollary 7.7], and for analytic $P^{2}(\mu)$-spaces the conclusion of the theorem was shown to be true in [ARS2] without the added hypothesis that $E \subseteq \Gamma(\mathcal{H})$.

The remainder of this paper is structured as follows. In Section 2 we provide some necessary background material about contraction operators $T$ on Hilbert spaces and we define the set $\Sigma(T)$, which has the property that whenever $\mathcal{H}$ satisfies (1.1) and (1.2), then $\Sigma(\mathcal{H})=\Sigma\left(M_{\zeta}, \mathcal{H}\right)$. In Section 3 we will specialize to spaces satisfying (1.1) and (1.2) and discuss the set $\Delta(\mathcal{H})$. We will show that $\Delta(\mathcal{H}) \subseteq \Sigma(\mathcal{H})$ a.e. and that $\mathcal{H}$ admits nontangential limits a.e. on $\Delta(\mathcal{H})$. In Section 4 we will construct examples of functions that show that $\mathcal{H}$ does not admit nontangential limits on $\partial \mathbb{D} \backslash \Delta(\mathcal{H})$. This will be done by use of interpolating sequences. In Section 5 we will prove Theorem 1.3, and in Section 6 we will discuss the index of invariant subspaces and prove Theorems 1.5, 1.7, and 1.8. Section 7 contains answers to Question 1.2 with conditions on the reproducing kernel $k$. We note that the results of Section 5 (i.e. Theorem 1.3) are used in the proof. In Section 8 we present some important examples, and Section 9 contains further remarks and a question. 


\section{Contractions in General}

In this section we will provide some necessary background material on the boundary set $\Sigma(\mathcal{H})$. Recall that a contraction operator on a Hilbert space is called absolutely continuous if the spectral measure of the minimal unitary dilation is absolutely continuous with respect to Lebesgue measure on $\partial \mathbb{D}$; see $[\mathrm{NF}$. The results of this section hold for all absolutely continuous contractions $T$ acting on an abstract separable Hilbert space $\mathcal{H}$, but will be applied later with $T=M_{\zeta}$ or $T=M_{\zeta}^{*}$.

Let $\mathcal{H}$ be a separable complex Hilbert space with norm denoted by $\|\cdot\|$, and let $T \in \mathcal{B}(\mathcal{H})$ be a contraction operator on $\mathcal{H}$. We start by noting a simple identity that will be used repeatedly. If $x \in \mathcal{H}$ and $\lambda \in \mathbb{D}$, then

$$
\begin{aligned}
& \left(1-|\lambda|^{2}\right)\left\|(1-\bar{\lambda} T)^{-1} x\right\|^{2} \\
& =\operatorname{Re}\left\langle(I+\bar{\lambda} T)(I-\bar{\lambda} T)^{-1} x, x\right\rangle-\left\|D \bar{\lambda}(I-\bar{\lambda} T)^{-1} x\right\|^{2},
\end{aligned}
$$

where we have used $D \geq 0$ to denote the defect operator satisfying $D^{2}=I-T^{*} T$. Identity (2.1) can be verified by a straightforward calculation and for complex numbers $\lambda, z$ it is analogous to the following identity:

$$
\frac{1-|\lambda|^{2}}{|1-\bar{\lambda} z|^{2}}=\frac{1-|\bar{\lambda} z|^{2}}{|1-\bar{\lambda} z|^{2}}-|\lambda|^{2} \frac{1-|z|^{2}}{|1-\bar{\lambda} z|^{2}}=\operatorname{Re} \frac{1+\bar{\lambda} z}{1-\bar{\lambda} z}-|\lambda|^{2} \frac{1-|z|^{2}}{|1-\bar{\lambda} z|^{2}} \text {. }
$$

Next we consider the following construction. Since $\|T\| \leq 1$ we have $T^{* n} T^{n} \rightarrow$ $A^{2}$ as $n \rightarrow \infty$ in the strong operator topology, for some positive operator $A \in \mathcal{B}(\mathcal{H})$. For $x \in \mathcal{H}$ set $\|x\|_{*}=\|A x\|=\lim _{n \rightarrow \infty}\left\|T^{n} x\right\|$. Then if $\mathcal{A}=\operatorname{ker} A^{\perp},\|\cdot\|_{*}$ defines an inner product norm on $\mathcal{A}$, and we use $\mathcal{H}_{*}$ to denote the completion of $\mathcal{A}$ with respect to $\|\cdot\|_{*}$. Let $P_{\mathcal{A}}$ be the orthogonal projection of $\mathcal{H}$ onto $\mathcal{A}$ and for $x \in \mathcal{A}$ set $S x=P_{\mathcal{A}} T x$. Since $T^{*} P_{\mathcal{A}} A^{2} P_{\mathcal{A}} T=T^{*} A^{2} T=A^{2}$ the linear transformation $S$ extends to be an isometric operator on $\mathcal{H}_{*}$. Let $U$ be the minimal unitary extension of $S$ acting on some Hilbert space $\mathcal{K}, \mathcal{H}_{*} \subseteq \mathcal{K}$. Then since $\mathcal{A}^{\perp}$ is invariant for $T$ we have for all polynomials $p$ and $q$ and all $x, y \in \mathcal{H}$,

$$
\begin{aligned}
\langle p(T) x, q(T) y\rangle_{*} & =\langle A p(T) x, A q(T) y\rangle \\
& =\left\langle A P_{\mathcal{A}} p(T) x, A P_{\mathcal{A}} q(T) y\right\rangle \\
& =\left\langle p(U) P_{\mathcal{A}} x, q(U) P_{\mathcal{A}} y\right\rangle_{*} \\
& =\int_{\partial \mathbb{D}} p(z) \overline{q(z)} d\left\langle E(z) P_{\mathcal{A}} x, P_{\mathcal{A}} y\right\rangle_{*} .
\end{aligned}
$$

Here we have used $E$ to denote the spectral measure of $U$.

Lemma 2.1. If $T$ is an absolutely continuous contraction acting on $\mathcal{H}$, then the spectral measure $E$ of the unitary operator $U$ is absolutely continuous.

Proof. Since $U$ is the minimal unitary extension of the isometric operator $S$ acting on $\mathcal{H}_{*}$, and since $\mathcal{A}$ is dense in $\mathcal{H}_{*}$, it suffices to show that for every $x \in \mathcal{A}$, the measure $\langle E(\cdot) x, x\rangle_{*}$ is absolutely continuous. Let $V$ be the minimal unitary dilation of $T$ and let $\tilde{E}$ denote the spectral measure of $V$. Then, if $p$ is a polynomial and if 
$x \in \mathcal{A}$, we have

$$
\begin{aligned}
\int_{\partial \mathbb{D}}|p(z)|^{2} d\langle E(z) x, x\rangle_{*} & =\|p(T) x\|_{*}^{2} \leq\|p(T) x\|^{2} \\
& \leq\|p(V) x\|^{2}=\int_{\partial \mathbb{D}}|p(z)|^{2} d\langle\tilde{E}(z) x, x\rangle .
\end{aligned}
$$

The lemma follows, because $\tilde{E}$ is absolutely continuous.

We will now assume that $T$ is an absolutely continuous contraction, and if $x, y \in$ $\mathcal{H}$ we will write $w_{x, y}$ for the Radon-Nikodým derivative of the absolutely continuous measure $\left\langle E(\cdot) P_{\mathcal{A}} x, P_{\mathcal{A}} y\right\rangle_{*}$ with respect to normalized Lebesgue measure on $\partial \mathbb{D}$, i.e.,

$$
d\left\langle E(z) P_{\mathcal{A}} x, P_{\mathcal{A}} y\right\rangle_{*}=w_{x, y}(z) \frac{|d z|}{2 \pi} .
$$

As we mentioned in the Introduction there is another way to think about the operator $U$ and its spectral measure. To explain this, let $T$ and $\mathcal{H}$ be as above and let $B$ be the minimal coisometric extension of $T$ acting on some Hilbert space $\mathcal{L}$, i.e., the operator such that $B^{*}$ is the minimal isometric dilation of $T^{*}$. By the Wold decomposition theorem $B=S^{*} \oplus R$ with respect to $\mathcal{L}=\mathcal{L}_{1} \oplus \mathcal{L}_{2}$, where $S^{*}$ is a backward unilateral shift of some multiplicity and $R$ is a unitary operator. For $i=1,2$, let $P_{i}$ denote the projection onto $\mathcal{L}_{i}$, and let $F$ denote the spectral measure of $R$. Then it is clear that for all $x \in \mathcal{H}$ we have $P_{2} T x=R P_{2} x, P_{1} T x=S^{*} P_{1} x$ and $\|x\|_{*}=\left\|P_{\mathcal{A}} x\right\|_{*}=\left\|P_{2} x\right\| \|_{\mathcal{L}}$. Thus for $x, y \in \mathcal{H}$ and all polynomials $p$ and $q$ we have

$$
\begin{aligned}
\int_{\partial \mathbb{D}} p(z) \overline{q(z)} d\left\langle E(z) P_{\mathcal{A}} x, P_{\mathcal{A}} y\right\rangle_{*} & =\langle p(T) x, q(T) y\rangle_{*}=\left\langle P_{2} p(T) x, P_{2} q(T) y\right\rangle_{\mathcal{L}} \\
& =\left\langle p(R) P_{2} x, q(R) P_{2} y\right\rangle_{\mathcal{L}} \\
& =\int_{\partial \mathbb{D}} p(z) \overline{q(z)} d\left\langle F(z) P_{2} x, P_{2} y\right\rangle_{\mathcal{L}},
\end{aligned}
$$

and the measures $\left\langle E(\cdot) P_{\mathcal{A}} x, P_{\mathcal{A}} y\right\rangle_{*}$ and $\left\langle F(\cdot) P_{2} x, P_{2} y\right\rangle_{\mathcal{L}}$ agree. It follows that for every $x \in \mathcal{H}$ and for almost every $z \in \partial \mathbb{D}$ the nontangential limit of

$$
\begin{aligned}
\left(1-|\lambda|^{2}\right)||(I-\bar{\lambda} R)^{-1} P_{2} x \|_{\mathcal{L}}^{2} & =\int_{\partial \mathbb{D}} \frac{1-|\lambda|^{2}}{|1-\bar{\lambda} w|^{2}} d\left\langle F(w) P_{2} x, P_{2} x\right\rangle_{\mathcal{L}} \\
& =\int_{\partial \mathbb{D}} \frac{1-|\lambda|^{2}}{|1-\bar{\lambda} w|^{2}} d\left\langle E(w) P_{\mathcal{A}} x, P_{\mathcal{A}} x\right\rangle_{*}
\end{aligned}
$$

at $z$ exists and equals $w_{x, x}(z)$. In particular, we notice that $w_{x, x}=0$, whenever $\left\|T^{n} x\right\| \rightarrow 0$ as $n \rightarrow \infty$.

Now let $x_{0} \in \mathcal{K}$ be a separating vector for $U$ (see Co, p.282) and write $W$ for the Radon-Nikodým derivative of the measure $\left\langle E(\cdot) x_{0}, x_{0}\right\rangle_{*}$. This measure is a scalar-valued spectral measure for $E$ ( $[\mathrm{Co}$, p. 286). We define $\Sigma(T)$ to be the set of Lebesgue points of $\{z \in \partial \mathbb{D}: W(z)>0\}$. It is easy to see that $\Sigma(T)$ does not depend on the choice of the separating vector $x_{0}$. In the almost everywhere sense $\Sigma(T)$ is the smallest set that carries $E$, and it is clear that for every $x \in \mathcal{H}$ we have $\left\{z \in \partial \mathbb{D}: w_{x, x}(z)>0\right\} \subseteq \Sigma(T)$ a.e.

We point out again that in the literature on absolutely continuous contractions typically the notation $R_{*}$ and $\Sigma_{*}(T)$ has been used for what we called $R$ and $\Sigma(T)$. 
The following lemma will be one of our main tools. For the case where $T$ is multiplication by $\zeta$ on some $P^{2}(\mu)$ - or $L^{2}(\mu)$-space with support $\mu \subseteq \overline{\mathbb{D}}$ the lemma was proved in $[\mathrm{KT}$. Also see ARS1, where a connection to Littlewood's theorem on subharmonic functions was pointed out.

Lemma 2.2. Let $T \in \mathcal{B}(\mathcal{H})$ be an absolutely continuous contraction, and let $x \in \mathcal{H}$. Then with the notation as above we have

$$
\operatorname{nt}_{\lambda \rightarrow z} \lim _{\lambda \rightarrow}\left(1-|\lambda|^{2}\right)\left\|(I-\bar{\lambda} T)^{-1} x\right\|^{2}=w_{x, x}(z)
$$

for a.e. $z \in \partial \mathbb{D}$.

In particular, nt- $\lim _{\lambda \rightarrow z}\left(1-|\lambda|^{2}\right)\left\|(1-\bar{\lambda} T)^{-1} x\right\|^{2}=0$ for a.e. $z \in \partial \mathbb{D} \backslash \Sigma(T)$, and, if $T^{n} x \rightarrow 0$, then nt- $\lim _{\lambda \rightarrow z}\left(1-|\lambda|^{2}\right)\left\|(1-\bar{\lambda} T)^{-1} x\right\|^{2}=0$ a.e.

Proof. As above we let $S^{*} \oplus R$ be the minimal coisometric extension of $T$ acting on $\mathcal{L}_{1} \oplus \mathcal{L}_{2}$. Thus, for $x \in \mathcal{H}$ we have

$$
\left\|(I-\bar{\lambda} T)^{-1} x\right\|^{2}=\left\|\left(I-\bar{\lambda} S^{*}\right)^{-1} P_{1} x\right\|_{\mathcal{L}}^{2}+\left\|(I-\bar{\lambda} R)^{-1} P_{2} x\right\|_{\mathcal{L}}^{2}
$$

where $S^{*}$ is a backward unilateral shift of some multiplicity, $R$ is unitary, and $P_{i}$ is the projection onto $\mathcal{L}_{i}, i=1,2$. It now follows from (2.3) that it suffices to show that for every $y \in \mathcal{L}_{1}$ we have $\left(1-|\lambda|^{2}\right)\left\|\left(I-\bar{\lambda} S^{*}\right)^{-1} y\right\|^{2} \rightarrow 0$ nontangentially a.e. That is probably well known, but since we do not have a reference we include a brief proof.

If $k \geq 1$ and $y \in \operatorname{ker} S^{* k}$, then

$$
\left(1-|\lambda|^{2}\right)\left\|\left(I-\bar{\lambda} S^{*}\right)^{-1} y\right\|^{2}=\left(1-|\lambda|^{2}\right)|| \sum_{n=0}^{k-1} \bar{\lambda}^{n} S^{* n} y\left\|^{2} \leq\left(1-|\lambda|^{2}\right) k^{2}\right\| y \|^{2} \rightarrow 0
$$

as $|\lambda| \rightarrow 1$. Thus, the result is true for a dense set of $y$ 's.

We set $v(\lambda)=\operatorname{Re}\left\langle\left(I+\bar{\lambda} S^{*}\right)\left(I-\bar{\lambda} S^{*}\right)^{-1} y, y\right\rangle$. Then $v$ is a positive harmonic function with $v(0)=\|y\|^{2}$, and it follows from identity (2.1) that the nontangential maximal function $N_{y}(z)$ of $\left(1-|\lambda|^{2}\right)\left\|\left(1-\bar{\lambda} S^{*}\right)^{-1} y\right\|^{2}$ is bounded by the nontangential maximal function of $v$ and thus satisfies a weak-type estimate of the form

$$
\left|\left\{z \in \partial \mathbb{D}: N_{y}(z)>\varepsilon\right\}\right| \leq C \frac{\|y\|^{2}}{\varepsilon} .
$$

An elementary standard argument finishes the proof.

Lemma 2.3. Let $T$ be an absolutely continuous contraction acting on $\mathcal{H}$. Let $\mathcal{A}$, $\mathcal{H}_{*}, \mathcal{K}$, and $U$ be as above, and let $\mathcal{D}$ be a dense subset of $\mathcal{H}$.

Then for every $\varepsilon>0$ there is an $h \in \mathcal{D}$ such that

$$
\left|\Sigma(T) \backslash\left\{z \in \partial \mathbb{D}: w_{h, h}(z)>0\right\}\right|<\varepsilon .
$$

Proof. By Lemma 2.1 the spectral measure $E$ of $U$ is absolutely continuous. For $x \in \mathcal{K}$ write $W_{x}$ for the $L^{1}(\partial \mathbb{D})$-function such that $W_{x}(z)|d z|=d\langle E(z) x, x\rangle_{*}$. Let $\varepsilon>0$ and let $x \in \mathcal{K}$ be a separating vector for $U$. Then $\left\{z \in \partial \mathbb{D}: W_{x}(z)>0\right\}$ has full Lebesgue measure in $\Sigma(T)$. Hence there is a $\delta>0$ such that $\mid \Sigma(T) \backslash\{z \in \partial \mathbb{D}$ : $\left.W_{x}(z)>\delta\right\} \mid<\varepsilon / 2$. Set $V=\left\{z \in \partial \mathbb{D}: W_{x}(z)>\delta\right\}$. 
Since $U$ is the minimal unitary extension of $S$, the closed span of $U^{* n} \mathcal{H}_{*}, n \geq 0$ is dense in $\mathcal{K}$. Since $P_{\mathcal{A}} \mathcal{D}$ is dense in $\mathcal{H}_{*}$ it now follows that there is a sequence $h_{n} \in \mathcal{D}$ such that $U^{* n} P_{\mathcal{A}} h_{n} \rightarrow x$. Set $u_{n}=P_{\mathcal{A}} h_{n}$ and $V_{n}=\left\{z \in \partial \mathbb{D}: w_{h_{n}, h_{n}}(z)>0\right\} \cap V$. To finish the proof it will suffice to show that $\left|V \backslash V_{n}\right| \rightarrow 0$.

One easily checks that for every bounded Borel measurable function $\varphi$ one has

$$
\begin{aligned}
\left|\int \varphi w_{h_{n}, h_{n}} \frac{|d z|}{2 \pi}-\int \varphi W_{x}\right| d z|| & =\left|\int \varphi d\left\langle E u_{n}, u_{n}\right\rangle_{*}-\int \varphi d\langle E x, x\rangle_{*}\right| \\
& =\left|\int \varphi d\left\langle E U^{* n} u_{n}, U^{* n} u_{n}\right\rangle_{*}-\int \varphi d\langle E x, x\rangle_{*}\right| \\
& \leq\|\varphi\|_{\infty}\left(\left\|u_{n}\right\|_{*}+\|x\|_{*}\right)\left\|U^{* n} u_{n}-x\right\|_{*} .
\end{aligned}
$$

Furthermore we have $\int \chi_{V \backslash V_{n}} w_{h_{n}, h_{n}}|d z|=0$ for each $n$. Thus

$$
\begin{aligned}
\delta\left|V \backslash V_{n}\right| & \leq \int \chi_{V \backslash V_{n}} W_{x}|d z| \\
& =\left|\int \chi_{V \backslash V_{n}} w_{h_{n}, h_{n}} \frac{|d z|}{2 \pi}-\int \chi_{V \backslash V_{n}} W_{x}\right| d z|| \\
& \leq\left(\left\|u_{n}\right\|_{*}+\|x\|_{*}\right)|| U^{* n} u_{n}-x \|_{*} \rightarrow 0 .
\end{aligned}
$$

Lemma 2.4. Let $T$ be an absolutely continuous contraction acting on $\mathcal{H}$. If $x, y \in$ $\mathcal{H}$, then for a.e. $z \in \partial \mathbb{D}$ we have $\left|w_{x, y}(z)\right| \leq \sqrt{w_{x, x}(z)} \sqrt{w_{y, y}(z)}$.

Furthermore if $\left\{x_{n}\right\}_{n},\left\{y_{n}\right\}_{n} \subseteq \mathcal{H},\left\{x_{n}\right\}_{n} \rightarrow x,\left\{y_{n}\right\}_{n} \rightarrow y$, then there are subsequences $\left\{x_{n_{k}}\right\}_{k},\left\{y_{n_{k}}\right\}_{k}$ such that $w_{x_{n_{k}}, y_{n_{k}}}(z) \rightarrow w_{x, y}(z)$ for a.e. $z \in \partial \mathbb{D}$ as $k \rightarrow \infty$.

Proof. With the notation as above we have for any Borel set $K \subseteq \partial \mathbb{D}$,

$$
\begin{aligned}
\left|\left\langle E(K) P_{\mathcal{A}} x, P_{\mathcal{A}} y\right\rangle_{*}\right| & \leq\left\|E(K) P_{\mathcal{A}} x\right\|_{*}\left\|E(K) P_{\mathcal{A}} y\right\|_{*} \\
& =\sqrt{\left\langle E(K) P_{\mathcal{A}} x, P_{\mathcal{A}} x\right\rangle_{*}} \sqrt{\left\langle E(K) P_{\mathcal{A}} y, P_{\mathcal{A}} y\right\rangle_{*}} .
\end{aligned}
$$

Thus the first inequality follows for every $z \in \partial \mathbb{D}$ that is a Lebesgue point for $w_{x, y}, w_{x, x}$ and $w_{y, y}$.

We now note that $\left|w_{x_{n}, y_{n}}-w_{x, y}\right|=\left|w_{x_{n}-x, y_{n}-y}+w_{x, y_{n}-y}+w_{x_{n}-x, y}\right|$ and we conclude from the above inequality that it suffices to show that there is a subsequence $\left\{x_{n_{k}}\right\}_{k}$ of $\left\{x_{n}\right\}_{n}$ such that $w_{x_{n}-x, x_{n}-x} \rightarrow 0$ a.e. In fact, we may also assume that $x_{n} \rightarrow 0$. But then $w_{x_{n}, x_{n}} \rightarrow 0$ in $L^{1}(\partial \mathbb{D})$, because $\int_{\partial \mathbb{D}}\left|w_{x_{n}, x_{n}}(z)\right| \frac{|d z|}{2 \pi}=\left\|x_{n}\right\|_{*}^{2} \leq$ $\left\|x_{n}\right\|^{2}$. The result follows.

\section{Hilbert spaces of analytic functions where $M_{\zeta}$ is COntractive}

In this section we will assume that $\mathcal{H}$ is a nonzero Hilbert space of analytic functions satisfying only conditions (1.1) and (1.2). As before we shall write $k_{\lambda}(z)$ for the reproducing kernel of $\mathcal{H}$.

The first lemma is well known, but we include its brief proof for completeness.

Lemma 3.1. Let $\mathcal{H}$ be a Hilbert space of analytic functions satisfying conditions (1.1) and (1.2). Then $M_{\zeta}^{* n} \rightarrow 0$ in the strong operator topology.

Consequently for all $h \in \mathcal{H}$ we have $\left(1-|\lambda|^{2}\right)\left\|\left(I-\lambda M_{\zeta}^{*}\right)^{-1} h\right\|^{2} \rightarrow 0$ a.e. as $\lambda$ approaches $z \in \partial \mathbb{D}$ nontangentially. 
Proof. It is clear that $M_{\zeta}^{* n} k_{\lambda}=\bar{\lambda}^{n} k_{\lambda} \rightarrow 0$ for each $\lambda \in \mathbb{D}$. Hence $M_{\zeta}^{* n} h \rightarrow 0$ whenever $h$ is a finite linear combination of reproducing kernels. Thus the sequence $\left\{M_{\zeta}^{* n}\right\}$ is norm bounded and approaches zero on a dense set and the first part of the lemma follows immediately. The second part follows from Lemma 2.2.

It is obvious from Lemma 3.1 that $M_{\zeta}$ is completely nonunitary. Hence by a well-known theorem of Sz.-Nagy and Foiaş $\left[\mathrm{NF}, M_{\zeta}\right.$ is absolutely continuous, and it follows from Lemma 2.1 that the spectral measure $E$ of the unitary operator $U$ of Section 2 is absolutely continuous. We shall now write $\Sigma(\mathcal{H})$ for $\Sigma\left(M_{\zeta}\right)$, where $M_{\zeta}$ is supposed to act on $\mathcal{H}$.

Next we set up some standard notation. Let $0<\sigma<1$ and $z \in \partial \mathbb{D}$. Then we use $\Gamma_{\sigma}(z)$ to denote a Stolz region at $z \in \partial \mathbb{D}$, i.e., the interior of the convex hull of $z$ and the circle with center 0 and radius $\sigma$. Furthermore, for a closed set $E \subseteq \partial \mathbb{D}$ we let $\Omega_{E, \sigma}=\bigcup_{z \in E} \Gamma_{\sigma}(z)$. It is well known that $\Omega_{E, \sigma}$ is a simply connected domain bounded by a rectifiable Jordan curve. We say that a sequence $\left\{\lambda_{n}\right\}_{n \geq 0}$ in $\mathbb{D}$ converges to $z \in \partial \mathbb{D}$ nontangentially if $\lambda_{n} \rightarrow z$ as $n \rightarrow \infty$ and there exists $0<\sigma<1$ such that $\lambda_{n} \in \Gamma_{\sigma}(z)$ for all $n$. In the following we will usually keep $\sigma$ fixed and write $\Omega_{E}$ for $\Omega_{E, \sigma}$.

If $f \in \mathcal{H}$ and $\lambda \in \mathbb{D}$, then $|f(\lambda)| \leq|| f|||| k_{\lambda} \|$, so $\frac{|f(\lambda)|}{\left\|k_{\lambda}\right\|}=O(1)$ as $|\lambda| \rightarrow 1$. The following proposition says that one can do better if one is willing to neglect sets of measure 0 and let $\lambda \rightarrow \partial \mathbb{D}$ nontangentially.

Proposition 3.2. Let $\mathcal{H}$ be a Hilbert space of analytic functions on $\mathbb{D}$ satisfying conditions (1.1) and (1.2). Let $f \in \mathcal{H}$. Then

$$
\mathrm{nt}_{\lambda \rightarrow z} \frac{|f(\lambda)|^{2}}{\left(1-|\lambda|^{2}\right)|| k_{\lambda}||^{2}} \leq w_{f, f}(z) \text { for a.e. } z \in \partial \mathbb{D} .
$$

Consequently, $|f(\lambda)|=O\left(\sqrt{1-|\lambda|^{2}}|| k_{\lambda}||\right)$ a.e. as $\lambda$ approaches $z \in \partial \mathbb{D}$ nontangentially, and $|f(\lambda)|=o\left(\sqrt{1-|\lambda|^{2}}|| k_{\lambda}||\right)$ as $\lambda$ approaches a.e. $z \in \partial \mathbb{D} \backslash \Sigma(\mathcal{H})$.

Furthermore, if $\left\|\zeta^{n} f\right\| \rightarrow 0$ as $n \rightarrow \infty$, then we have $|f(\lambda)|=o\left(\sqrt{1-|\lambda|^{2}}|| k_{\lambda}||\right)$ a.e. as $\lambda$ approaches $z \in \partial \mathbb{D}$ nontangentially.

Proof. Let $\lambda \in \mathbb{D}$. Then since $\left\|M_{\zeta}\right\| \leq 1$ the function $(1-\bar{\lambda} \zeta)^{-1} f$ is in $\mathcal{H}$ and we have

$$
\begin{aligned}
|f(\lambda)| & \leq\left(1-|\lambda|^{2}\right)\left|\left\langle(1-\bar{\lambda} \zeta)^{-1} f, k_{\lambda}\right\rangle\right| \\
& \leq \sqrt{1-|\lambda|^{2}}||(1-\bar{\lambda} \zeta)^{-1} f\left\|\sqrt{1-|\lambda|^{2}}|| k_{\lambda}\right\| .
\end{aligned}
$$

The result now follows from Lemma 2.2 .

For $g$ in $\mathcal{H}, g \neq 0$ set

$$
\Delta_{g}(\mathcal{H})=\left\{z \in \partial \mathbb{D}: \underset{\lambda \rightarrow z}{n} \varlimsup_{\lambda \rightarrow z}\left(1-|\lambda|^{2}\right) \frac{\|\left. k_{\lambda}\right|^{2}}{|g(\lambda)|^{2}}<\infty\right\} .
$$

It will follow from the next proposition that the set $\Delta_{g}(\mathcal{H})$ is independent of the choice of $g$ if sets of measure 0 are neglected. Notice that it follows from Proposition 3.2 that for every nonzero $g \in \mathcal{H}$ we have $\Delta_{g}(\mathcal{H}) \subseteq\left\{z \in \partial \mathbb{D}: w_{g, g}(z)>0\right\} \subseteq \Sigma(\mathcal{H})$ a.e.

For an open set $\Omega$ in the complex plane with $0 \in \Omega$ we denote by $H^{2}(\Omega)$ the Hardy space on $\Omega$. This is the space of analytic functions on $\Omega$ with the property 
that $|f|^{2}$ has a harmonic majorant, and we take $\|f\|_{H^{2}(\Omega)}^{2}=v_{f}(0)$, where $v_{f}$ is the least harmonic majorant of $|f|^{2}$ on $\Omega$.

Proposition 3.3. Fix $0<\sigma<1$ and let $\mathcal{H}$ be a Hilbert space of analytic functions on $\mathbb{D}$ satisfying conditions (1.1) and (1.2).

If $g \in \mathcal{H}, g \neq 0$ and $\varepsilon>0$, then there is a finite Blaschke product $B$, a closed set $E \subseteq \Delta_{g}(\mathcal{H})$ with $\left|\Delta_{g}(\mathcal{H}) \backslash E\right|<\varepsilon$, and a $c>0$ such that for every $f \in \mathcal{H}$ we have $\frac{B f}{g} \in H^{2}\left(\Omega_{E}\right)$ and $\left\|\frac{B f}{g}\right\|_{H^{2}\left(\Omega_{E}\right)} \leq c\|f\|$.

Hence for every $f \in \mathcal{H}$ the function $f / g$ has nontangential limits a.e. on $\Delta_{g}(\mathcal{H})$ and $\Delta_{f}(\mathcal{H})=\Delta_{g}(\mathcal{H})$ a.e. whenever $f \neq 0$.

Proof. Let $g \in \mathcal{H}, g \neq 0$ and $\varepsilon>0$. Consider the sets $E_{n} \subset \Delta_{g}(\mathcal{H})$ consisting of those points $z \in \Delta_{g}(\mathcal{H})$ with nt- $\lim _{\lambda \rightarrow z}\left(1-|\lambda|^{2}\right) \frac{\left\|k_{\lambda}\right\|^{2}}{|g(\lambda)|^{2}} \leq n$ and with the property that for all $\lambda \in \Gamma_{\sigma}(z)$ with $|\lambda|>1-1 / n$ we have

$$
\left(1-|\lambda|^{2}\right) \frac{\|\left. k_{\lambda}\right|^{2}}{|g(\lambda)|^{2}}<2 n \text {. }
$$

Clearly $E_{n} \subseteq E_{n+1}$ and the set $\Delta_{g}(\mathcal{H}) \backslash \bigcup_{n \geq 1} E_{n}$ has measure zero. Thus we may choose a closed set $E \subset \Delta_{g}(\mathcal{H})$ with $\left|\Delta_{g}(\mathcal{H}) \backslash E\right|<\varepsilon$ such that $E \subset E_{n}$ for some $n \geq 1$. Note that $k_{\lambda} / g$ has at most a finite number of poles in $\Omega_{E}$, and hence there exists a finite Blaschke product $B$ such that the function $\lambda \rightarrow\left(1-|\lambda|^{2}\right)|B(\lambda)|^{2} \frac{\left\|k_{\lambda}\right\|^{2}}{|g(\lambda)|^{2}}$ is bounded in $\Omega_{E}$. If we let $c>0$ be an upper bound for this function, then as in the proof of Lemma 3.2 we obtain for all $\lambda \in \Omega_{E}$,

$$
\begin{aligned}
\frac{|B(\lambda) f(\lambda)|^{2}}{|g(\lambda)|^{2}} & =\left(1-|\lambda|^{2}\right)^{2}\left|\left\langle(1-\bar{\lambda} \zeta)^{-1} f, k_{\lambda}\right\rangle\right|^{2} \frac{|B(\lambda)|^{2}}{|g(\lambda)|^{2}} \\
& \leq\left(1-|\lambda|^{2}\right)||(1-\bar{\lambda} \zeta)^{-1} f \|^{2}\left(1-|\lambda|^{2}\right)|| k_{\lambda}||^{2} \frac{|B(\lambda)|^{2}}{|g(\lambda)|^{2}} \\
& \leq c\left(1-|\lambda|^{2}\right)||(1-\bar{\lambda} \zeta)^{-1} f \|^{2} .
\end{aligned}
$$

Recall from identity (2.1) that the function $\lambda \mapsto\left(1-|\lambda|^{2}\right)\left\|(1-\bar{\lambda} \zeta)^{-1} f\right\|^{2}$ is bounded in the unit disc by a positive harmonic function $v_{f}$ with $v_{f}(0)=\|f\|^{2}$. This implies that $B f / g \in H^{2}\left(\Omega_{E}\right)$ and $\left\|\frac{B f}{g}\right\|_{H^{2}\left(\Omega_{E}\right)} \leq c\|f\|$.

It now follows from Theorem 10.3 of $[\mathrm{Du}$ ] that for each $f \in \mathcal{H}$ the function $f / g$ has nontangential limits a.e. on $E$. Thus by letting $\varepsilon \rightarrow 0$ we see that $f / g$ has a nontangential limit $f / g(z)$ for a.e. $z \in \Delta_{g}(\mathcal{H})$. If $f \neq 0$, then for a.e. $z \in \Delta_{g}(\mathcal{H})$ we have

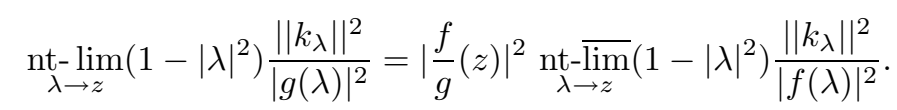

By Privalov's theorem this implies that $\Delta_{g}(\mathcal{H}) \subseteq \Delta_{f}(\mathcal{H})$ a.e. We obtain a.e. equality by reversing the roles of $f$ and $g$.

We shall from now on drop the subscript and write $\Delta(\mathcal{H})$ for the set of Lebesgue points of $\Delta_{g}(\mathcal{H})$, where $g$ is some nonzero function in $\mathcal{H}$. Thus we have proved the following corollary, which also is Theorem 1.1(a).

Corollary 3.4. Let $\mathcal{H}$ be a nonzero Hilbert space of analytic functions on $\mathbb{D}$ satisfying conditions (1.1) and (1.2). Then $\mathcal{H}$ admits nontangential limits on $\Delta(\mathcal{H})$. 
Note that Proposition 3.2 implies that for each $f \in \mathcal{H}$ the nontangential limit of $\frac{|f(\lambda)|^{2}}{\left(1-\left.|\lambda|^{2}|| k_{\lambda}\right|^{2}\right.}$ exists a.e. on $\partial \mathbb{D} \backslash \Sigma(\mathcal{H})$. It is of interest to note that the same conclusion holds on $\Delta(\mathcal{H})$.

Proposition 3.5. Let $\mathcal{H}$ be a nonzero Hilbert space of analytic functions on $\mathbb{D}$ satisfying conditions (1.1) and (1.2) and let $g \in \mathcal{H}$. Then for a.e. $z \in \Delta(\mathcal{H}) \cup$ $(\partial \mathbb{D} \backslash \Sigma(\mathcal{H}))$ the limit of $\frac{|g(\lambda)|^{2}}{\left(1-|\lambda|^{2}\right)|| k_{\lambda} \|^{2}}$ exists as $\lambda$ approaches $z$ nontangentially.

Proof. For some $\varepsilon>0$ let $E \subseteq \Delta(\mathcal{H})$, let the Blaschke product be $B$, and let $c>0$ be as in Proposition 3.3. It follows from the fundamental inequality (1.5) with $f=g$ and from Lemma 2.2 that nt- $\underline{\lim }_{\lambda \rightarrow z}\left(1-|\lambda|^{2}\right) \frac{\left\|k_{\lambda}\right\|^{2}}{|g(\lambda)|^{2}}>0$ for almost all $z \in \partial \mathbb{D}$. Since $\varepsilon>0$ was arbitrary it thus suffices to show that $H(\lambda)=|B(\lambda)|^{2}\left(1-|\lambda|^{2}\right) \frac{\left\|k_{\lambda}\right\|^{2}}{|g(\lambda)|^{2}}$ has nontangential limits a.e. on $E$. Note that it follows from the proof of Proposition 3.3 that $H(\lambda) \leq c$ for all $\lambda \in \Omega_{E}$.

Next we will apply some standard facts about positive definite sesquianalytic kernels. For some more background information we refer the reader to the first paragraph of Section 7. Since $M_{\zeta}$ is a contraction it follows that the function $(1-\bar{\lambda} z) k_{\lambda}(z)$ is a positive definite sesquianalytic kernel on $\mathbb{D} \times \mathbb{D}$. Thus, there are analytic functions $g_{n}$ on $\mathbb{D}$ such that $(1-\bar{\lambda} z) k_{\lambda}(z)=\sum_{n} \overline{g_{n}(\lambda)} g_{n}(z)$ for all $\lambda, z \in \mathbb{D}$. Let $\varphi$ be a conformal map from $\mathbb{D}$ onto $\Omega_{E}$ that fixes the origin. Since $\partial \Omega_{E}$ is a rectifiable Jordan curve, $\varphi$ extends to be continuous and 1-1 from $\overline{\mathbb{D}}$ onto $\overline{\Omega_{E}}$. We set $f_{n}(\alpha)=\frac{B g_{n}}{g}(\varphi(\alpha)), \alpha \in \mathbb{D}$. Now let $\mathcal{C}$ be a separable Hilbert space with orthonormal basis $\left\{e_{n}\right\}_{n}$ and define a $\mathcal{C}$-valued analytic function $F$ on $\mathbb{D}$ by $F(\alpha)=\sum_{n} f_{n}(\alpha) e_{n}, \alpha \in \mathbb{D}$. Then $\|F(\alpha)\|^{2}=\sum_{n}\left|f_{n}(\alpha)\right|^{2}=H(\varphi(\alpha)) \leq c$ for all $\alpha \in \mathbb{D}$. Thus $F$ is a bounded analytic function, so by the Hilbert space valued version of Fatou's Theorem (see e.g. p. 81 of $[\overline{R R}$ ), $F(\alpha)$ has nontangential norm-limits a.e. on $\partial \mathbb{D}$. This of course implies that $\|F(\alpha)\|^{2}$ has nontangential limits a.e. on $\partial \mathbb{D}$, and it now follows from the fact that $\partial \Omega_{E}$ is rectifiable that $H(\lambda)=\left\|F\left(\varphi^{-1}(\lambda)\right)\right\|^{2}$ has nontangential limits a.e. on $E$.

For later reference we record that the preceding proof actually shows a slightly stronger statement. Indeed, since for each $n$ the meromorphic function in $\mathbb{D}, B g_{n} / g$, is actually in $H^{\infty}\left(\Omega_{E}\right)$, and $f_{n} \in H^{\infty}(\mathbb{D})$, the nontangential limits $g_{n} / g(z)$ and $f_{n}(w)$ exist respectively for a.e. $z \in E$ and a.e. $w \in \partial \mathbb{D}$. Thus for a.e. $z \in E$ we have

$$
\sum_{n}\left|\frac{g_{n}}{g}(z)\right|^{2}=\sum_{n}\left|f_{n}\left(\varphi^{-1}(z)\right)\right|^{2}=\left.|| F\left(\varphi^{-1}(z)\right)\right|^{2}=\operatorname{nt}_{\lambda \rightarrow z} \lim _{\lambda \rightarrow z}\left(1-|\lambda|^{2}\right) \frac{\left\|k_{\lambda}\right\|^{2}}{|g(\lambda)|^{2}} .
$$

Since $\varepsilon>0$ was arbitrary this last identity actually holds for almost all $z \in \Delta(\mathcal{H})$, and the statement holds for every sequence of analytic functions $g_{n} \in \operatorname{Hol}(\mathbb{D})$ with $(1-\bar{\lambda} z) k_{\lambda}(z)=\sum_{n} \overline{g_{n}(\lambda)} g_{n}(z)$ for all $\lambda, z \in \mathbb{D}$.

\section{Boundary BeHaVior NeAR $\partial \mathbb{D} \backslash \Delta(\mathcal{H})$}

In this section we will prove Theorem 1.1 (b) together with a strengthened version that will be needed later. Thus we again assume that $\mathcal{H}$ is a space of analytic functions which satisfies (1.1) and (1.2). If $\Lambda \subseteq \mathbb{D}$, then we write $\operatorname{Ntl} \Lambda$ for the set of nontangential limit points of $\Lambda$, i.e. those $z \in \partial \mathbb{D}$ for which there is a sequence of points $\left\{\lambda_{n}\right\}$ in $\Lambda$ that converges to $z$ and lies entirely in some Stolz angle $\Gamma_{\sigma}(z)$. 
Recall from [BSZ that $\Lambda$ is called dominating for $\partial \mathbb{D}$ if and only if Ntl $\Lambda$ has full measure in $\partial \mathbb{D}$.

Let $\Lambda \subseteq \mathbb{D}$ be a finite or infinite sequence of distinct points such that $\left\|k_{\lambda}\right\| \neq 0$ for all $\lambda \in \Lambda$. We say that $\Lambda$ is interpolating for $\mathcal{H}$ if the linear transformation $T_{\Lambda, \mathcal{H}}$ defined by

$$
T_{\Lambda, \mathcal{H}} f=\left\{f(\lambda) /\left\|k_{\lambda}\right\|\right\}_{\lambda \in \Lambda}
$$

maps $\mathcal{H}$ into and onto $l_{\Lambda}^{2}$, the space of complex-valued square summable sequences indexed by $\Lambda$. If $\Lambda$ is interpolating for $\mathcal{H}$, then it follows from the closed graph theorem and the open mapping theorem that there is a constant $M>0$ such that for all sequences $a=\left\{a_{\lambda}\right\} \in l_{\Lambda}^{2}$ there exists $f \in \mathcal{H}$ with $T_{\Lambda, \mathcal{H}} f=a$ and

$$
\frac{1}{M}\|f\|^{2} \leq\left\|T_{\Lambda, \mathcal{H}} f\right\|^{2} \leq M\|f\|^{2} .
$$

We call any such constant $M$ an interpolation constant for $\Lambda$. Of course, if $N$ is finite, then a sequence of $N$ distinct points in $\mathbb{D}$ is interpolating whenever the corresponding set $\left\{k_{\lambda}\right\}$ is linearly independent. In the following we will need to keep track of the interpolation constants for various finite sequences $\Lambda$. Since the space $\mathcal{H}$ will be fixed, we will just write $T_{\Lambda}$ for $T_{\Lambda, \mathcal{H}}$.

Theorem 4.1. Let $\mathcal{H}$ be a Hilbert space of analytic functions on $\mathbb{D}$ satisfying conditions (1.1) and (1.2).

(a) If $g \in \mathcal{H}, g \neq 0$, then there exists a function $f \in \mathcal{H}$ and a discrete set $\Lambda \subseteq \mathbb{D}$ such that $\operatorname{Ntl} \Lambda=\partial \mathbb{D} \backslash \Delta(\mathcal{H})$ a.e. and such that $\left|\frac{f}{g}(\lambda)\right| \rightarrow \infty$ as $|\lambda| \rightarrow 1, \lambda \in \Lambda$. In particular, there is no subset $E$ of $\partial \mathbb{D} \backslash \Delta(\mathcal{H})$ of positive measure such that $\mathcal{H}$ admits nontangential limits on $E$.

(b) If $H^{\infty}$ is contained and dense in $\mathcal{H}$, then there is a sequence $\Lambda \subseteq \mathbb{D}$ that is interpolating for $\mathcal{H}$ and satisfies $\mathrm{Ntl} \Lambda=\partial \mathbb{D} \backslash \Delta(\mathcal{H})$ a.e.

Note that in the special case where $H^{\infty}$ is contained and dense in $\mathcal{H}$ the statement (b) of the theorem implies (a) with $f=1$ and a certain $g$. This follows because interpolating sequences are zero sequences. Thus (b) implies that there is a nonzero function $g \in \mathcal{H}$ such that $g(\lambda)=0$ for every $\lambda \in \Lambda$. Then neither $g$ nor $1 / g$ can have nontangential limits on any subset of $\partial \mathbb{D} \backslash \Delta(\mathcal{H})$ of positive measure.

For the construction that is required for Theorem 4.1 we will need a series of definitions and lemmas.

Let $g \in \mathcal{H}, g \neq 0$, and write $Z(g)=\{\lambda \in \mathbb{D}: g(\lambda)=0\}$. For $\lambda \in \mathbb{D} \backslash Z(g)$ define $L(\lambda)=\sqrt{1-|\lambda|^{2}} \frac{\| k_{\lambda}||}{|g(\lambda)|}$. If $g\left(\lambda_{0}\right)=0$, then set $L\left(\lambda_{0}\right)=\lim _{\lambda \rightarrow \lambda_{0}} L(\lambda)$. Thus the function $L$ is continuous as a function on $\mathbb{D}$ with values in $[0, \infty]$, and the definition of $\Delta(\mathcal{H})$ implies that for a.e. $z \in \partial \mathbb{D} \backslash \Delta(\mathcal{H})$ there is a sequence $\left\{\lambda_{n}\right\}_{n \in \mathbb{N}}$ that converges to $z$ nontangentially and such that $L\left(\lambda_{n}\right) \rightarrow \infty$.

For $\lambda \in \mathbb{D}$ and $\sigma>0$ we write $I_{\lambda, \sigma}$ for the open interval in $\partial \mathbb{D}$ that is centered at $\lambda /|\lambda|$ and has Lebesgue measure $\left|I_{\lambda, \sigma}\right|=2 \pi \sigma(1-|\lambda|)$. Note that a simple geometric argument shows that a sequence $\left\{\lambda_{n}\right\}_{n \geq 0} \subseteq \mathbb{D},\left|\lambda_{n}\right| \rightarrow 1$ converges nontangentially to $z \in \partial \mathbb{D}$ if and only if there is a $\sigma>0$ such that $z \in \bigcap_{n \geq N_{0}} I_{\lambda_{n}, \sigma}$ for sufficiently large $N_{0}$. Furthermore if we fix $\sigma>0$, then it is a standard fact that there is a set $E_{\sigma}$ of full measure in $\partial \mathbb{D} \backslash \Delta(\mathcal{H})$ such that for every $z \in E_{\sigma}$ there is a sequence $\left\{\lambda_{n}\right\}_{n \in \mathbb{N}}$ that converges to $z$ with $z \in \bigcap_{n \geq 0} I_{\lambda_{n}, \sigma}$ and such that $L\left(\lambda_{n}\right) \rightarrow \infty$. 
Since $L$ is continuous and $Z(g)$ is discrete, there will even be such a sequence with $g\left(\lambda_{n}\right) \neq 0$ for each $n$.

For the remainder of this section we will now fix a $\sigma>0$ as above and write $I_{\lambda}=I_{\lambda, \sigma}$ and $(\partial \mathbb{D} \backslash \Delta(\mathcal{H}))_{\sigma}=E_{\sigma}$. Furthermore we use the notation $\hat{I}_{\lambda}$ to denote the open interval in $\partial \mathbb{D}$ that has the same center as $I_{\lambda}$ and 3 times its length.

Definition 4.2. Let $E \subseteq \partial \mathbb{D}$ be closed. A finite subset $F \subseteq \mathbb{D}$ is called a V-set for $E$ if the collection $\left\{I_{\lambda}\right\}_{\lambda \in F}$ consists of mutually disjoint intervals and if $\left\{\hat{I}_{\lambda}\right\}_{\lambda \in F}$ covers $E$. We say that $F$ is a $\mathrm{V}$-set if it is a $\mathrm{V}$-set for some closed set $E$, i.e., if the intervals $\left\{I_{\lambda}\right\}_{\lambda \in F}$ are mutually disjoint.

If $F$ is a V-set, then we write $L(F)=\inf \{L(\lambda): \lambda \in F\}$ and $\mathcal{M}_{F}=$ $\operatorname{span}\left\{k_{\lambda}: \lambda \in F\right\}$.

Lemma 4.3. Let $0<r<1, L>0$, and $E \subseteq(\partial \mathbb{D} \backslash \Delta(\mathcal{H}))_{\sigma}$ be closed.

Then there is a $V$-set $F \subseteq \mathbb{D} \backslash Z(g)$ for $E$ such that $F \cap r \mathbb{D}=\emptyset$ and such that $L(F)>L$.

Proof. The definition of the set $(\partial \mathbb{D} \backslash \Delta(\mathcal{H}))_{\sigma}$ implies that

$$
E \subseteq(\partial \mathbb{D} \backslash \Delta(\mathcal{H}))_{\sigma} \subseteq \bigcup_{|\lambda| \geq r, L(\lambda)>L, g(\lambda) \neq 0} I_{\lambda} .
$$

Thus the compactness of $E$ implies that there are finitely many points $\lambda$ in $\mathbb{D} \backslash r \mathbb{D}$ such that the corresponding $\operatorname{arcs} I_{\lambda}$ cover $E, L(\lambda)>L$, and $g(\lambda) \neq 0$. The conclusion now follows from a Vitali-type covering lemma (see $\underline{\mathrm{Ru}}$, Lemma 7.3, p.137).

Lemma 4.4. If $K>0$ and $u_{1}, u_{2}, \ldots, u_{n}$ are unit vectors in $\mathcal{H}$ such that whenever $a_{1}, \ldots, a_{n}, b_{1}, \ldots, b_{n} \in \mathbb{C}$ with $\left|b_{j}\right| \leq\left|a_{j}\right|, j=1, \ldots, n$, then $\left\|\sum_{j=1}^{n} b_{j} u_{j}\right\| \leq$ $K\left\|\sum_{j=1}^{n} a_{j} u_{j}\right\|$, then for all $a_{1}, \ldots, a_{n} \in \mathbb{C}$ we have

$$
\frac{1}{K}\left(\sum_{j=1}^{n}\left|a_{j}\right|^{2}\right)^{1 / 2} \leq\left\|\sum_{j=1}^{n} a_{j} u_{j}\right\| \leq K\left(\sum_{j=1}^{n}\left|a_{j}\right|^{2}\right)^{1 / 2} .
$$

This is a part of the well-known Koethe-Toeplitz Theorem. The elementary proof can be based on a lemma by W. Orlicz (see [Ni], p.159) or, similarly, on the fact that for any $a_{1}, \ldots, a_{n} \in \mathbb{C}$ and unit vectors $u_{1}, u_{2}, \ldots, u_{n} \in \mathcal{H}$ one can inductively construct $b_{1}, \ldots, b_{n} \in \mathbb{C}$ with $\left|b_{j}\right|=\left|a_{j}\right|, j=1, \ldots, n$ and $\|\left.\sum_{j=1}^{n} b_{j} u_{j}\right|^{2}=\sum_{j=1}^{n}\left|b_{j}\right|^{2}$.

Lemma 4.5. There is a constant $K=K(\sigma)>0$ such that every $V$-set $F \subseteq \mathbb{D}$ is interpolating for $\mathcal{H}$ with interpolation constant $K$.

More specifically, the following is true:

If $F=\left\{\lambda_{1}, \lambda_{2}, \ldots, \lambda_{n}\right\} \subseteq \mathbb{D}$ is a $V$-set, and if $a_{1}, a_{2}, \ldots, a_{n} \in \mathbb{C}$, then

$$
\frac{1}{K}\left(\sum_{j=1}^{n}\left|a_{j}\right|^{2}\right)^{1 / 2} \leq\left\|\sum_{j=1}^{n} a_{j} \frac{k_{\lambda_{j}}}{\left\|k_{\lambda_{j}}\right\|}\right\| \leq K\left(\sum_{j=1}^{n}\left|a_{j}\right|^{2}\right)^{1 / 2} .
$$

Proof. We consider the adjoint operator $T_{F}^{*}: l_{F}^{2} \rightarrow \mathcal{H}$. It is clear from condition (4.1) that we must show that $T_{F}^{*}$ is bounded by $K$ and bounded below by $1 / K$. This condition is easily seen to be precisely what is stated in the second part of the lemma. 
Since the intervals $\left\{I_{\lambda}\right\}_{\lambda \in F}$ are mutually disjoint it follows that there is a $\gamma>0$ dependent only on $\sigma$ such that $\left|\frac{\lambda_{i}-\lambda_{j}}{1-\overline{\lambda_{i}} \lambda_{j}}\right| \geq \gamma$ for all $i \neq j$ and such that for every Carleson square $S_{h}$ we have $\sum_{\lambda_{j} \in S_{h}}\left(1-\left|\lambda_{j}\right|\right) \leq \frac{1}{\gamma} h$. Thus by Carleson's $H^{\infty}$ interpolation theorem there is a constant $K>0$ dependent only on $\sigma$ such that whenever $c_{1}, c_{2}, \ldots, c_{n} \in \mathbb{C}$, then there is a bounded analytic function $\varphi$ on $\mathbb{D}$ with $\overline{\varphi\left(\lambda_{j}\right)}=c_{j}, j=1,2, \ldots, n$ and $\|\varphi\|_{\infty} \leq K \sup _{j}\left\{\left|c_{j}\right|\right\}$; see Ga.

We shall now conclude the proof of Lemma 4.5 by verifying the hypothesis of Lemma 4.4 with the above $K$ and $u_{j}=\frac{k_{\lambda_{j}}}{\left\|k_{\lambda_{j}}\right\|}, j=1, \ldots, n$. Indeed, if $a_{1}, \ldots, a_{n}$ and $b_{1}, \ldots, b_{n} \in \mathbb{C}$ are given with $\left|b_{j}\right| \leq\left|a_{j}\right|, j=1, \ldots, n$, then for $j=1, \ldots, n$ we choose $c_{j}$ so that $c_{j} a_{j}=b_{j}$. Hence there exists a $\varphi \in H^{\infty}$ such that $\overline{\varphi\left(\lambda_{j}\right)}=c_{j}$, $j=1,2, \ldots, n$ and $\|\varphi\|_{\infty} \leq K$, and we have

$$
\left\|\sum_{j=1}^{n} b_{j} \frac{k_{\lambda_{j}}}{\left\|k_{\lambda_{j}}\right\|}\right\|=\left\|M_{\varphi}^{*} \sum_{j=1}^{n} a_{j} \frac{k_{\lambda_{j}}}{\left\|k_{\lambda_{j}}\right\|}\right\| \leq\left\|M_{\varphi}^{*}\right\|\left\|\sum_{j=1}^{n} a_{j} \frac{k_{\lambda_{j}}}{\left\|k_{\lambda_{j}}\right\|}\right\| \leq K\left\|\sum_{j=1}^{n} a_{j} \frac{k_{\lambda_{j}}}{\left\|k_{\lambda_{j}}\right\|}\right\| .
$$

In the remainder of this section, $K$ will always denote the constant from Lemma 4.5.

Lemma 4.6. If $F \subseteq \mathbb{D}$ is any $V$-set, and if $\left\{\zeta_{\lambda}\right\}_{\lambda \in F} \subseteq \partial \mathbb{D}$, then there is an $f \in M_{F}$ such that $\|f\| \leq K \sqrt{2 / \sigma}$, arg $f(\lambda)=\arg \zeta_{\lambda}$, and $\left|\frac{f}{g}(\lambda)\right| \geq L(F)$ for all $\lambda \in F$.

Proof. Let $\left\{e_{\lambda}\right\}_{\lambda \in F}$ denote the standard orthonormal basis of $l_{F}^{2}$ satisfying $e_{\lambda}(\mu)=$ $\delta_{\lambda, \mu}$ for $\lambda, \mu \in F$. By Lemma 4.5 the operator $T_{F}$ is invertible when restricted to $M_{F}$ with $\left\|\left(T_{F} \mid M_{F}\right)^{-1}\right\| \leq K$. For $\lambda \in F$ we set $f_{\lambda}=\left(T_{F} \mid M_{F}\right)^{-1} e_{\lambda}$, so that $f_{\lambda}(\mu)=\delta_{\lambda, \mu}\left\|k_{\lambda}\right\|$.

We set $f=\sum_{\lambda \in F} \sqrt{1-|\lambda|^{2}} \zeta_{\lambda} f_{\lambda}$. Then

$$
\begin{aligned}
\|f\|^{2} & =\left\|\left(T_{F} \mid M_{F}\right)^{-1} \sum_{\lambda \in F} \sqrt{1-|\lambda|^{2}} \zeta_{\lambda} e_{\lambda}\right\| \\
& \leq K\left\|\sum_{\lambda \in F} \sqrt{1-|\lambda|^{2}} \zeta_{\lambda} e_{\lambda}\right\|=K\left(\sum_{\lambda \in F} 1-|\lambda|^{2}\right)^{1 / 2} \\
& \leq K \sqrt{2 /(2 \pi \sigma})\left(\sum_{\lambda \in F}\left|I_{\lambda}\right|\right)^{1 / 2} \leq K \sqrt{2 / \sigma}
\end{aligned}
$$

For $\lambda \in F$ we see that $f(\lambda)=\sqrt{1-|\lambda|^{2}} \zeta_{\lambda}\left\|k_{\lambda}\right\|$; hence $\arg f(\lambda)=\arg \zeta_{\lambda}$, and $\left|\frac{f}{g}(\lambda)\right|=L(\lambda) \geq L(F)$.

We are now ready to prove Theorem 4.1 (a).

Proof of Theorem 4.1 (a). Let $\left\{E_{n}\right\}$ be a monotonically increasing sequence of closed subsets of $(\partial \mathbb{D} \backslash \Delta(\mathcal{H}))_{\sigma}$ such that $\bigcup_{n=1}^{\infty} E_{n}=\partial \mathbb{D} \backslash \Delta(\mathcal{H})$ a.e.. It is possible to find such sets by the remarks preceding Definition 4.2 .

We will inductively construct a sequence of $\mathrm{V}$-sets $\left\{F_{n}\right\}_{n \geq 1}$, a sequence of radii $\left\{r_{n}\right\}_{n \geq 0} \subseteq[0,1)$, a sequence of positive coefficients $\left\{a_{n}\right\}_{n \geq 1}$, and two sequences of functions $\left\{f_{n}\right\}_{n \geq 1}$ and $\left\{h_{n}\right\}_{n \geq 1}$ such that $r_{0}=0$ and for each $n \geq 1$ :

(4.2) $F_{n}$ is a V-set for $E_{n}$ with $r_{n-1} \leq \inf \left\{|\lambda|: \lambda \in F_{n}\right\}$ and $F_{n} \cap Z(g)=\emptyset$,

(4.3) $0<a_{n} \leq 2^{-n}, r_{n}=\frac{1+\sup \left\{|\lambda|: \lambda \in \bar{F}_{n}\right\}}{2}$, 
(4.4) $f_{n} \in M_{F_{n}}$ and $\left\|f_{n}\right\| \leq K \sqrt{2 / \sigma}$,

(4.5) $h_{n}=\sum_{k=1}^{n} a_{k} f_{k}$,

(4.6) if $\lambda \in F_{n}$, then $\left|\frac{h_{n}}{g}(\lambda)\right| \geq n$,

(4.7) if $1 \leq j<n$ and $\lambda \in F_{j}$, then $a_{n}\left|\frac{f_{n}}{g}(\lambda)\right| \leq 2^{-n}$.

We will first show how to use these functions and $\mathrm{V}$-sets to prove Theorem 4.1 (a). We let $\Lambda=\bigcup_{n>1} F_{n}$ and $f=\lim _{n \rightarrow \infty} h_{n}=\sum_{k=1}^{\infty} a_{k} f_{k}$; this converges in $\mathcal{H}$ by (4.3), (4.4), and (4.5). If $n \geq 1$ and $\lambda \in \Lambda$ with $|\lambda|>r_{n}$, then by (4.2) $\lambda \in F_{j}$ for some $j>n$ and $\left|\frac{f(\lambda)}{g(\lambda)}\right| \geq \frac{\left|h_{j}(\lambda)\right|}{|g(\lambda)|}-\sum_{k=j+1}^{\infty} a_{k} \frac{\left|f_{k}(\lambda)\right|}{|g(\lambda)|} \geq j-2^{-j} \geq n-1$. Thus $\left|\frac{f}{g}(\lambda)\right| \rightarrow \infty$ as $|\lambda| \rightarrow 1, \lambda \in \Lambda$. Furthermore the fact that $\mathrm{Ntl} \Lambda=\partial \mathbb{D} \backslash \Delta(\mathcal{H})$ a.e. follows from the fact that every $z \in \bigcup_{n=1}^{\infty} E_{n}$ lies in the intersection of infinitely many intervals of the type $\hat{I}_{\lambda}$. Indeed, if $z \in E_{n}$, then for each $k \geq n, F_{k}$ is a $\mathrm{V}$-set for $E_{n}$. Hence there is a $\lambda_{k} \in F_{k}$ such that $z \in \hat{I}_{\lambda_{k}}$. It is clear that $\left\{\lambda_{k}\right\}_{k \geq n} \subseteq \Lambda$ and that $\left\{\lambda_{k}\right\}_{k \geq n}$ converges to $z$ nontangentially.

We now start with the inductive procedure. Set $a_{1}=1 / 2$ and use Lemma 4.3 to choose a $\mathrm{V}$-set $F_{1} \subseteq \mathbb{D} \backslash Z(g)$ for $E_{1}$ with $L\left(F_{1}\right) \geq 2$. Then we use Lemma 4.6 to choose $f_{1} \in M_{F_{1}}$ with $\left\|f_{1}\right\| \leq K \sqrt{2 / \sigma}, f_{1}(\lambda)>0$ and $\left|\frac{f_{1}}{g}(\lambda)\right| \geq L\left(F_{1}\right)$ for all $\lambda \in F_{1}$. Set $h_{1}=a_{1} f_{1}$ and $r_{1}=\frac{1+\sup \left\{|\lambda|: \lambda \in F_{1}\right\}}{2}$. It is clear that (4.2)-(4.6) hold for $n=1$. (4.7) is vacuous; thus it holds trivially.

Next suppose that $n \geq 1$ and that for $j=1, . ., n$ we have constructed V-sets $F_{j}$, radii $r_{j} \in[0,1)$, positive coefficients $a_{j}$, and functions $f_{j}$ and $h_{j}$ such that (4.2)-(4.7) hold for all $j$ between 1 and $n$.

Set $M_{n}=\sup \left\{|| k_{\zeta} \|:|z| \leq r_{n}\right\}+1$ and $\varepsilon=\inf \left\{|g(\lambda)|: \lambda \in F_{j}\right.$ for some $j, 1 \leq$ $j \leq n\}$. Note that $\varepsilon>0$ since $\bigcup_{j=1}^{n} F_{j}$ is finite and does not intersect $Z(g)$ by (4.2). Choose $0<a_{n+1}<2^{-(n+1)}$ such that $a_{n+1}<2^{-(n+1)} \frac{\varepsilon}{M_{n} K} \sqrt{\sigma / 2}$, and use Lemma 4.3 to select a V-set $F_{n+1} \subseteq \mathbb{D} \backslash r_{n} \mathbb{D}$ with $F_{n+1} \cap Z(g)=\emptyset$ and $L\left(F_{n+1}\right) \geq \frac{n+1}{a_{n+1}}$. For $\lambda \in F_{n+1}$ set $\zeta_{\lambda}=h_{n}(\lambda) /\left|h_{n}(\lambda)\right|$ if $h_{n}(\lambda) \neq 0$ and $\zeta_{\lambda}=1$ otherwise. Hence Lemma 4.6 implies the existence of a function $f_{n+1} \in M_{F_{n+1}}$ with $\left\|f_{n+1}\right\| \leq K \sqrt{2 / \sigma}$, $\left|h_{n}(\lambda)+a_{n+1} f_{n+1}(\lambda)\right|=\left|h_{n}(\lambda)\right|+\left|a_{n+1} f_{n+1}(\lambda)\right|$ and $\left|\frac{f_{n+1}}{g}(\lambda)\right| \geq L\left(F_{n+1}\right)$ for all $\lambda \in F_{n+1}$. We set $h_{n+1}=h_{n}+a_{n+1} f_{n+1}$, and note that it follows immediately from the choice of our $(n+1)$-st generation parameters that conditions (4.2)-(4.5) are satisfied. Furthermore for $\lambda \in F_{n+1}$ we have

$$
\left|\frac{h_{n+1}}{g}(\lambda)\right|=\frac{\left|h_{n}(\lambda)+a_{n+1} f_{n+1}(\lambda)\right|}{|g(\lambda)|} \geq a_{n+1}\left|\frac{f_{n+1}}{g}(\lambda)\right| \geq a_{n+1} L\left(F_{n+1}\right) \geq n+1,
$$

so (4.6) holds. Finally, we verify condition (4.7). If $1 \leq j \leq n$ and $\lambda \in F_{j}$, then

$$
a_{n+1} \frac{\left|f_{n+1}(\lambda)\right|}{|g(\lambda)|} \leq a_{n+1} \frac{|| k_{\lambda}|||| f_{n+1}||}{\varepsilon} \leq a_{n+1} \frac{M_{n} K}{\varepsilon} \sqrt{2 / \sigma} \leq 2^{-(n+1)} .
$$

In order to prove Theorem 4.1 (b) we need one more lemma. We will now assume that $H^{\infty}$ is contained and dense in $\mathcal{H}$ and we will fix $g=1$ in the definition of the function $L$. Thus $L(\lambda)=\sqrt{1-|\lambda|^{2}}|| k_{\lambda}||$.

Lemma 4.7. Suppose $H^{\infty}$ is contained and dense in $\mathcal{H}$. Let $\delta>0$ and let $F \subseteq \mathbb{D}$ be a $V$-set.

Then there is $L>0$ such that whenever $G \subseteq \mathbb{D}$ is a $V$-set with $L(G)>L$, then $|\langle u, v\rangle| \leq \delta|| u|||| v \|$ for all $u \in M_{F}$ and $v \in M_{G}$. 
Proof. Let $m$ denote the cardinality of $F$. For each $\mu \in F$ choose $f_{\mu} \in H^{\infty}$ such that $\left\|f_{\mu}-\frac{k_{\mu}}{\left\|k_{\mu}\right\|}\right\| \leq \frac{\delta}{2 K \sqrt{m}}$, where $K$ is the constant from Lemma 4.5. Set $M=\max \left\{\left\|f_{\mu}\right\|_{H^{\infty}}: \mu \in F\right\}$ and choose $L=\frac{2 M \sqrt{m} K^{2}}{\delta} \sqrt{2 / \sigma}$.

Now let $G \subseteq \mathbb{D}$ be a V-set with $L(G)>L$, and let $u=\sum_{\mu \in F} a_{\mu} \frac{k_{\mu}}{\left\|k_{\mu}\right\|} \in M_{F}$ and $v=\sum_{\lambda \in G} b_{\lambda} \frac{k_{\lambda}}{\left\|k_{\lambda}\right\|} \in M_{G}$. Then

$$
\begin{aligned}
|\langle u, v\rangle| & \leq\left|\left\langle\sum_{\mu \in F} a_{\mu}\left(\frac{k_{\mu}}{\left\|k_{\mu}\right\|}-f_{\mu}\right), v\right\rangle\right|+\left|\left\langle\sum_{\mu \in F} a_{\mu} f_{\mu}, \sum_{\lambda \in G} b_{\lambda} \frac{k_{\lambda}}{\left\|k_{\lambda}\right\|}\right\rangle\right| \\
& \left.\leq\left(\sum_{\mu \in F}\left|a_{\mu}\right|^{2}\right)^{1 / 2}\left(\sum_{\mu \in F} \| \frac{k_{\mu}}{\left\|k_{\mu}\right\|}-f_{\mu}\right) \|^{2}\right)^{1 / 2}|| v \|+\sum_{\mu \in F, \lambda \in G} \frac{\left|a_{\mu} \| b_{\lambda}\right|}{\left\|k_{\lambda}\right\|}\left|f_{\mu}(\lambda)\right| \\
& \leq K\|u\| \frac{\delta}{2 K \sqrt{m}} \sqrt{m}\|v\|+\frac{M}{L(G)} \sqrt{m}\left(\sum_{\mu \in F}\left|a_{\mu}\right|^{2}\right)^{1 / 2} \sum_{\lambda \in G}\left|b_{\lambda}\right| \sqrt{1-|\lambda|^{2}} \\
& \leq \frac{\delta}{2}\|u\|\|v\|+\frac{M K}{L}\|u\| K\|v\|\left(\sum_{\lambda \in G}\left(1-|\lambda|^{2}\right)\right)^{1 / 2} \\
& \leq \delta\|u\|\|v\|,
\end{aligned}
$$

where the last inequality followed from the definition of $L$ and because we have $\sum_{\lambda \in G}\left(1-|\lambda|^{2}\right) \leq 2 / \sigma$ for every V-set $G$.

Proof of Theorem 4.1 (b). As in the proof of Theorem 4.1 (a) we let $\left\{E_{n}\right\}$ be a monotonically increasing sequence of closed subsets of $(\partial \mathbb{D} \backslash \Delta(\mathcal{H}))_{\sigma}$ such that $\bigcup_{n=1}^{\infty} E_{n}=\partial \mathbb{D} \backslash \Delta(\mathcal{H})$ a.e., and we will inductively construct a sequence of V-sets $\left\{F_{n}\right\}_{n>1}$, where for each $n, F_{n}$ is a $\mathrm{V}$-set for $E_{n}$. Then as before it is clear that $\Lambda=\bigcup_{n \geq 1} F_{n}$ satisfies Ntl $\Lambda=\partial \mathbb{D} \backslash \Delta(\mathcal{H})$ a.e. We need to construct the V-sets $F_{n}$ in such a way that $\Lambda$ is interpolating for $\mathcal{H}$.

Choose a sequence of positive numbers $\left\{\varepsilon_{i}\right\}$ such that $\sum_{i} \varepsilon_{i}^{2} \leq 1 / 2$. We will construct the sequence $\left\{F_{n}\right\}$ such that

$$
\left|\left\langle u_{i}, u_{j}\right\rangle\right| \leq \varepsilon_{i} \varepsilon_{j}|| u_{i}|||| u_{j} \| \text { whenever } i \neq j \text { and } u_{i} \in M_{F_{i}}, u_{j} \in M_{F_{j}} .
$$

We start by using Lemma 4.3 to choose a $\mathrm{V}$-set $F_{1}$ for $E_{1}$. Next we suppose that $n \geq 1$ and that we have already chosen $\mathrm{V}$-sets $F_{1}, \ldots, F_{n}$ satisfying (4.8) for all $i \neq j, 1 \leq i, j \leq n$. For $1 \leq i \leq n$ set $\delta_{i}=\varepsilon_{i} \varepsilon_{n+1}$ and let $L_{i}$ be the constant obtained when we apply Lemma 4.7 with $F_{i}$ and $\delta_{i}$. Thus we can use Lemma 4.3 to choose a V-set $F_{n+1}$ for $E_{n+1}$ with $L\left(F_{n+1}\right) \geq L_{i}$ for each $i=1, \ldots, n$. It is then clear from the conclusion of Lemma 4.7 that (4.8) holds for $F_{1}, \ldots, F_{n+1}$, and we have shown that a sequence of $\mathrm{V}$-sets can be chosen which satisfies (4.8).

We fix such a sequence $\left\{F_{n}\right\}$ and set $\Lambda=\bigcup_{n \geq 1} F_{n}$. For $i \geq 1$ let $u_{i} \in M_{F_{i}}$. Then for each $n$ we have

$$
\begin{aligned}
\left|\left\|\sum_{i=1}^{n} u_{i}\right\|^{2}-\sum_{i=1}^{n}\left\|u_{i}\right\|^{2}\right| & \leq \sum_{i \neq j}^{n}\left|\left\langle u_{i}, u_{j}\right\rangle\right| \leq \sum_{i \neq j}^{n} \varepsilon_{i} \varepsilon_{j}\left\|u_{i}\right\|\left\|\mid u_{j}\right\| \\
& \leq\left(\sum_{i=1}^{n} \varepsilon_{i}\left\|u_{i}\right\|\right)^{2} \leq \sum_{i=1}^{n} \varepsilon_{i}^{2} \sum_{i=1}^{n}\left\|u_{i}\right\|^{2} \leq 1 / 2 \sum_{i=1}^{n}\left\|u_{i}\right\|^{2} .
\end{aligned}
$$


This implies that

$$
\frac{1}{2} \sum_{i=1}^{n}\left\|u_{i}\right\|^{2} \leq\left\|\sum_{i=1}^{n} u_{i}\right\|^{2} \leq \frac{3}{2} \sum_{i=1}^{n}\left\|u_{i}\right\|^{2}
$$

We now combine this with Lemma 4.5 to obtain

$$
\frac{1}{2 K} \sum_{i}\left|a_{i}\right|^{2} \leq\left\|\sum_{i} a_{i} \frac{k_{\lambda_{i}}}{\left\|k_{\lambda_{i}}\right\|}\right\|^{2} \leq \frac{3}{2 K} \sum_{i}\left|a_{i}\right|^{2}
$$

whenever the $\lambda_{i}$ are finitely many distinct points in $\Lambda$ and $a_{i} \in \mathbb{C}$. This shows that the adjoint $T_{\Lambda}^{*}$ of the operator from (4.1) is bounded and bounded below; hence (4.1) is satisfied and $\Lambda$ is interpolating for $\mathcal{H}$.

\section{A condition for the a.e. equality of $\Sigma(\mathcal{H})$ and $\Delta(\mathcal{H})$}

In this section we will prove Theorem 1.3. Thus we shall assume that $\mathcal{H}$ is a Hilbert space of analytic functions on $\mathbb{D}$ which satisfies (1.1)-(1.4).

We shall need the following simple lemma. Recall from Section 2 that if $f, h \in \mathcal{H}$, then $w_{f, h}$ is the $L^{1}$-function satisfying $d\left\langle E(\cdot) P_{\mathcal{A}} f, P_{\mathcal{A}} h\right\rangle_{*}=w_{f, h} \frac{|d z|}{2 \pi}$ (see Section 2 for the definitions of $\mathcal{A}, E$, and $\left.\langle\cdot, \cdot\rangle_{*}\right)$. In particular, equation (2.2) implies that if $p$ and $q$ are polynomials, then we have $\int_{\partial \mathbb{D}} p(z) \overline{q(z)} w_{f, h}(z) \frac{|d z|}{2 \pi}=\langle p f, q h\rangle_{*}$. An easy approximation argument shows that this equality remains true if $p, q \in$ $H^{\infty}$. If $\varphi$ and $\psi$ are another two $H^{\infty}$-functions, then we can apply this identity with $p \varphi$ and $q \psi$ and obtain $\int_{\partial \mathbb{D}} p(z) \overline{q(z)} \varphi(z) \overline{\psi(z)} w_{f, h}(z) \frac{|d z|}{2 \pi}=\langle p \varphi f, q \psi h\rangle_{*}=$ $\int_{\partial \mathbb{D}} p(z) \overline{q(z)} w_{\varphi f, \psi h}(z) \frac{|d z|}{2 \pi}$. It follows that $\varphi \bar{\psi} w_{f, h}=w_{\varphi f, \psi h}$.

For $\lambda \in \mathbb{D}$ we use $P_{\lambda}$ to denote the Poisson kernel, $P_{\lambda}(z)=\frac{1-|\lambda|^{2}}{|z-\lambda|^{2}}, z \in \partial \mathbb{D}$.

Lemma 5.1. Let $f, h \in \mathcal{H}$ and $\lambda \in \mathbb{D}$. Then for any Blaschke product $B$ we have

$$
\left|\int_{\partial \mathbb{D}} P_{\lambda}(z) \bar{z} B(z) w_{f, h}(z) \frac{|d z|}{2 \pi}\right|^{2} \leq\left(1-|\lambda|^{2}\right)\left\|\frac{f}{1-\bar{\lambda} \zeta}\right\|^{2} \int_{\partial \mathbb{D}} P_{\lambda}(z) w_{h, h}(z) \frac{|d z|}{2 \pi} .
$$

Proof. By Lemma 2.4 we have

$$
\begin{aligned}
\mid \int_{\partial \mathbb{D}} & \left.P_{\lambda}(z) \bar{z} B(z) w_{f, h}(z) \frac{|d z|}{2 \pi}\right|^{2} \\
& \leq\left(\int_{\partial \mathbb{D}} P_{\lambda}(z) \sqrt{w_{f, f}(z)} \sqrt{w_{h, h}(z) \frac{|d z|}{2 \pi}}\right)^{2} \\
& \leq\left(\int_{\partial \mathbb{D}} P_{\lambda}(z) w_{f, f}(z) \frac{|d z|}{2 \pi}\right)\left(\int_{\partial \mathbb{D}} P_{\lambda}(z) w_{h, h}(z) \frac{|d z|}{2 \pi}\right) \\
& =\left(1-|\lambda|^{2}\right)\left\|\frac{f}{1-\bar{\lambda} \zeta}\right\|_{*}^{2}\left(\int_{\partial \mathbb{D}} P_{\lambda}(z) w_{h, h}(z) \frac{|d z|}{2 \pi}\right) \\
& \leq\left(1-|\lambda|^{2}\right)\left\|\frac{f}{1-\bar{\lambda} \zeta}\right\|^{2} \int_{\partial \mathbb{D}} P_{\lambda}(z) w_{h, h}(z) \frac{|d z|}{2 \pi} .
\end{aligned}
$$


Recall from equation (1.9) the definition of the set $\Gamma(\mathcal{H})$.

Theorem 5.2. If $\mathcal{H}$ satisfies (1.1)-(1.4), then

$$
\Delta(\mathcal{H}) \cap \Gamma(\mathcal{H})=\Sigma(\mathcal{H}) \cap \Gamma(\mathcal{H}) \text { a.e. }
$$

and for every $g \in \mathcal{H}$ we have

$$
w_{g, g}(z)=\operatorname{nt}_{\lambda \rightarrow z} \lim _{\lambda \rightarrow z} \frac{|g(\lambda)|^{2}}{\left(1-|\lambda|^{2}\right)|| k_{\lambda} \|^{2}} \quad \text { for a.e. } z \in \Gamma(\mathcal{H}) \cup(\partial \mathbb{D} \backslash \Sigma(\mathcal{H}))
$$

Furthermore, for every $f, g \in \mathcal{H}, g \neq 0$ we have $w_{f, f}=\left|\frac{f}{g}\right|^{2} w_{g, g}$ a.e. on $\Delta(\mathcal{H}) \cap \Gamma(\mathcal{H})$ and if $\Sigma(\mathcal{H}) \subseteq \Gamma(\mathcal{H})$ a.e., then

$$
\|f\|_{*}^{2}=\left\|w_{f, f}\right\|_{L^{1}}=\int_{\Delta(\mathcal{H})}\left|\frac{f}{g}(z)\right|^{2} w_{g, g}(z) \frac{|d z|}{2 \pi} .
$$

Here $\frac{f}{g}(z)$ denotes the nontangential limit of the meromorphic function $\frac{f}{g}$.

Proof. For $f \in \mathcal{H}$ we set $\|f\|_{1}^{2}=\|f\|^{2}-\frac{1}{2}\|f\|_{*}^{2}$. Then $\|\cdot\|_{1}$ defines a Hilbert space norm on $\mathcal{H}$ that is equivalent to the original norm with $\frac{1}{2}\|f\|^{2} \leq\|f\|_{1}^{2} \leq\|f\|^{2}$ for all $f \in \mathcal{H}$. Furthermore, if we use $T^{*}$ to denote the adjoint of the operator $M_{\zeta}$ with respect to the norm $\|\cdot\|_{1}$, then since the space $\mathcal{H}$ satisfies conditions (1.1) and (1.2) with respect to $\|\cdot\|_{1}$ we conclude from Lemma 3.1 that $T^{* n} \rightarrow 0$ in the strong operator topology.

Let $\varepsilon>0$ and let $H$ be a closed subset of $\Sigma(\mathcal{H}) \cap \Gamma(\mathcal{H})$ with

$$
|(\Sigma(\mathcal{H}) \cap \Gamma(\mathcal{H})) \backslash H|<\varepsilon
$$

and such that for some fixed $0<\sigma<1$ and some $c>0$ we have

$$
\left\|\frac{\zeta-\lambda}{1-\bar{\lambda} \zeta} f\right\| \geq c\|f\| \quad \text { for all } f \in \mathcal{H} \text { and all } \lambda \in \Omega_{H}=\Omega_{H, \sigma}
$$

Let $\mathcal{D}$ denote the set of finite linear combinations of reproducing kernels of $(\mathcal{H},\|\cdot\|)$, and let $h \in \mathcal{D}$. Then $h=\sum_{i=1}^{n} a_{i} k_{\lambda_{i}}, \lambda_{1}, \ldots, \lambda_{n} \in \mathbb{D}$, and we let $B$ be a finite Blaschke product with zeros $\lambda_{1}, \ldots, \lambda_{n}$.

The choice of $B$ implies that $\langle B g, h\rangle=0$ for each $g \in \mathcal{H}$. Furthermore it follows from conditions (1.3) and (1.4) that for each $f, g \in \mathcal{H}, \lambda \in \mathbb{D}$ with $g(\lambda) \neq 0$ we have $f-\frac{f}{g}(\lambda) g \in(\zeta-\lambda) \mathcal{H}$. Hence for all $f, g \in \mathcal{H}$ we have

$$
\left(1-|\lambda|^{2}\right)\left\langle B \frac{f-\frac{f}{g}(\lambda) g}{(\zeta-\lambda)(1-\bar{\lambda} \zeta)}, h\right\rangle=0
$$


In the following we shall always let $\lambda \in \Omega_{H}$ with $g(\lambda) \neq 0$. Then it follows from (5.2) and the definition of $\|\cdot\|_{1}$ that

$$
\begin{aligned}
\left(1-|\lambda|^{2}\right)\left|\left\langle B \frac{f-\frac{f}{g}(\lambda) g}{(\zeta-\lambda)(1-\bar{\lambda} \zeta)}, h\right\rangle_{*}\right| \\
=2\left(1-|\lambda|^{2}\right)\left|\left\langle B \frac{f-\frac{f}{g}(\lambda) g}{(\zeta-\lambda)(1-\bar{\lambda} \zeta)}, h\right\rangle_{1}\right| \\
\quad=2\left(1-|\lambda|^{2}\right)\left|\left\langle B \frac{f-\frac{f}{g}(\lambda) g}{\zeta-\lambda},\left(I-\lambda T^{*}\right)^{-1} h\right\rangle_{1}\right| \\
\quad \leq 2\left(1-|\lambda|^{2}\right)\left\|B \frac{f-\frac{f}{g}(\lambda) g}{\zeta-\lambda}\right\|\left\|_{1}\right\|\left(I-\lambda T^{*}\right)^{-1} h \|_{1} \\
\quad \leq 2\left(1-|\lambda|^{2}\right)\left\|\frac{f-\frac{f}{g}(\lambda) g}{\zeta-\lambda}\right\|\left\|\left(I-\lambda T^{*}\right)^{-1} h\right\|_{1} \\
\quad \leq \frac{2}{c}\left(1-|\lambda|^{2}\right)\left\|\frac{f-\frac{f}{g}(\lambda) g}{1-\bar{\lambda} \zeta}\right\|\left\|\left(I-\lambda T^{*}\right)^{-1} h\right\|_{1} \quad \text { by } \quad(5.1) \\
\quad \leq c(\lambda)\left(1-|\lambda|^{2}\right)^{1 / 2}\left(\left\|\frac{f}{1-\bar{\lambda} \zeta}\right\|+\left|\frac{f}{g}(\lambda)\right|\left\|\frac{g}{1-\bar{\lambda} \zeta}\right\|\right) .
\end{aligned}
$$

Here we have written $c(\lambda)=\frac{2}{c} \sqrt{1-|\lambda|^{2}}||\left(I-\lambda T^{*}\right)^{-1} h \|_{1}$, and we note that it follows from Lemma 2.2 that for a.e. $z \in \partial \mathbb{D}, c(\lambda) \rightarrow 0$ as $\lambda$ approaches $z$ nontangentially.

Now write $h_{\lambda}=\frac{f-\frac{f}{g}(\lambda) g}{\zeta-\lambda} \in \mathcal{H}$, and recall the notation from Section 2 with the absolutely continuous contraction $M_{\zeta}$ in the norm $\|\cdot\|$. Since $B$ is a finite Blaschke product $B(U)$ is defined via the functional calculus for unitary operators. Also, the definitions imply that $(U-\lambda) P_{\mathcal{A}} h_{\lambda}=P_{\mathcal{A}}\left(M_{\zeta}-\lambda\right) h_{\lambda}=P_{\mathcal{A}}\left(f-\frac{f}{g}(\lambda) g\right)$. Thus $P_{\mathcal{A}} h_{\lambda}=(U-\lambda)^{-1} P_{\mathcal{A}}\left(f-\frac{f}{g}(\lambda) g\right)$. Hence

$$
\begin{aligned}
&\langle B\left.\frac{f-\frac{f}{g}(\lambda) g}{(\zeta-\lambda)(1-\bar{\lambda} \zeta)}, h\right\rangle_{*}=\left\langle P_{\mathcal{A}} B \frac{f-\frac{f}{g}(\lambda) g}{(\zeta-\lambda)(1-\bar{\lambda} \zeta)}, P_{\mathcal{A}} h\right\rangle_{*} \\
&=\left\langle B(U)(1-\bar{\lambda} U)^{-1}(U-\lambda)^{-1} P_{\mathcal{A}}\left(f-\frac{f}{g}(\lambda) g\right), P_{\mathcal{A}} h\right\rangle_{*} \\
&=\left\langle B(U)(1-\bar{\lambda} U)^{-1}(U-\lambda)^{-1} P_{\mathcal{A}} f, P_{\mathcal{A}} h\right\rangle_{*} \\
& \quad-\frac{f}{g}(\lambda)\left\langle B(U)(1-\bar{\lambda} U)^{-1}(U-\lambda)^{-1} P_{\mathcal{A}} g, P_{\mathcal{A}} h\right\rangle_{*} \\
&=\int_{\partial \mathbb{D}} \frac{B(z)}{(z-\lambda)(1-\bar{\lambda} z)} d\left\langle E(z) P_{\mathcal{A}} f, P_{\mathcal{A}} h\right\rangle_{*} \\
& \quad-\frac{f}{g}(\lambda) \int_{\partial \mathbb{D}} \frac{B(z)}{(z-\lambda)(1-\bar{\lambda} z)} d\left\langle E(z) P_{\mathcal{A}} g, P_{\mathcal{A}} h\right\rangle_{*} \\
&=\int_{\partial \mathbb{D}} \frac{\bar{z} B(z)}{|z-\lambda|^{2}} w_{f, h}(z) \frac{|d z|}{2 \pi}-\frac{f}{g}(\lambda) \int_{\partial \mathbb{D}} \frac{\bar{z} B(z)}{|z-\lambda|^{2}} w_{g, h}(z) \frac{|d z|}{2 \pi} .
\end{aligned}
$$


Thus

$$
\begin{aligned}
\left(1-|\lambda|^{2}\right) \mid\langle & \left.B \frac{f-\frac{f}{g}(\lambda) g}{(\zeta-\lambda)(1-\bar{\lambda} \zeta)}, h\right\rangle_{*} \mid \\
& =\left|\int_{\partial \mathbb{D}} P_{\lambda}(z) \bar{z} B(z)\left(w_{f, h}(z)-\frac{f}{g}(\lambda) w_{g, h}(z)\right) \frac{|d z|}{2 \pi}\right| \\
& \geq\left|\frac{f}{g}(\lambda) \int_{\partial \mathbb{D}} P_{\lambda}(z) \bar{z} B(z) w_{g, h}(z) \frac{|d z|}{2 \pi}\right|-\left|\int_{\partial \mathbb{D}} P_{\lambda}(z) \bar{z} B(z) w_{f, h}(z) \frac{|d z|}{2 \pi}\right| .
\end{aligned}
$$

We combine the previous two inequalities, rearrange, and obtain

$$
\begin{aligned}
\left|\frac{f}{g}(\lambda)\right| & \left(\left|\int_{\partial \mathbb{D}} P_{\lambda}(z) \bar{z} B(z) w_{g, h}(z) \frac{|d z|}{2 \pi}\right|-c(\lambda) \sqrt{1-|\lambda|^{2}}|| \frac{g}{1-\bar{\lambda} \zeta} \|\right) \\
& \leq\left|\int_{\partial \mathbb{D}} P_{\lambda}(z) \bar{z} B(z) w_{f, h}(z) \frac{|d z|}{2 \pi}\right|+c(\lambda) \sqrt{1-|\lambda|^{2}} \mid \frac{f}{1-\bar{\lambda} \zeta} \| \\
& \leq \sqrt{1-|\lambda|^{2}}|| \frac{f}{1-\bar{\lambda} \zeta} \|\left(\sqrt{\int_{\partial \mathbb{D}} P_{\lambda}(z) w_{h, h}(z) \frac{|d z|}{2 \pi}}+c(\lambda)\right),
\end{aligned}
$$

where the last inequality followed from Lemma 5.1. We now take nontangential limits to obtain for a.e. $z \in H$,

$$
\mathrm{nt}_{\lambda \rightarrow z}\left|\frac{f}{g}(\lambda)\right|^{2}\left|w_{g, h}(z)\right|^{2} \leq w_{f, f}(z) w_{h, h}(z)
$$

and we note that the inequality is valid for all $f, g \in \mathcal{H}, g \neq 0$, and $h \in \mathcal{D}$. We also note that we used the convention that $\infty \cdot 0=0$. Furthermore, since $\varepsilon>0$ was arbitrary it follows that (5.6) holds for a.e. $z \in \Sigma(\mathcal{H}) \cap \Gamma(H)$.

Let $E=\left\{z \in \Gamma(\mathcal{H}): w_{h, h}(z)>0\right\} \subseteq \Sigma(\mathcal{H})$ and use (5.6) with $g=h \neq 0$. Then we see that for a.e. $z \in E$ the right-hand side of (5.3) converges to 0 as $\lambda$ approaches $z$ nontangentially. It thus follows from the first equality of (5.4) combined with (5.3) that for a.e. $z \in E$ the nontangential limit of $(f / h)(\lambda)$ exists as $\lambda \rightarrow z$ and $w_{f, h}(z)=\frac{f}{h}(z) w_{h, h}(z)$. This is true for all $f \in \mathcal{H}$; thus $\mathcal{H}$ admits nontangential limits on $E$. By Theorem 4.1 this implies that $E \subseteq \Delta(\mathcal{H})$ a.e. Finally, Lemma 2.3 implies that by choosing $h \in \mathcal{D}$ appropriately we may make the measure of $(\Sigma(\mathcal{H}) \cap \Gamma(\mathcal{H})) \backslash E$ as small as we wish. Hence $\Sigma(\mathcal{H}) \cap \Gamma(\mathcal{H}) \subseteq \Delta(\mathcal{H})$ a.e. and since we already know the other inclusion from Section 3 this shows that $\Sigma(\mathcal{H}) \cap \Gamma(\mathcal{H})=\Delta(\mathcal{H}) \cap \Gamma(\mathcal{H})$ a.e.

Another application of (5.4), (5.3), taking nontangential limits, and letting $\varepsilon \rightarrow 0$ shows that for all $f, g \in \mathcal{H}, g \neq 0, h \in \mathcal{D}$ we have $w_{f, h}(z)=\frac{f}{g}(z) w_{g, h}(z)$ for a.e. $z \in \Sigma(\mathcal{H}) \cap \Gamma(\mathcal{H})$. In fact, since $\mathcal{D}$ is dense in $\mathcal{H}$, Lemma 2.4 implies that the previous statement holds for all $h \in \mathcal{H}$. Hence we see that $w_{f, f}(z)=\frac{f}{g}(z) w_{g, f}(z)=$ $\frac{f}{g}(z) \overline{w_{f, g}(z)}=\left|\frac{f}{g}(z)\right|^{2} w_{g, g}(z)$ for a.e. $z \in \Sigma(\mathcal{H}) \cap \Gamma(\mathcal{H})$ whenever $f, g \in \mathcal{H}, g \neq 0$.

The fundamental inequality (1.5) and Lemma 2.2 together with Privalov's theorem now imply that for every nonzero $f \in \mathcal{H}$ we have $w_{f, f}(z)>0$ for a.e. $z \in \Sigma(\mathcal{H}) \cap \Gamma(\mathcal{H})$.

Next we go back to inequality (5.5) and apply it with $f=(1-\bar{\lambda} \zeta) k_{\lambda}, g \in \mathcal{H}$, $g \neq 0$, and $h \in \mathcal{D}$. Recall that it is valid for $\lambda \in \Omega_{H}$, where the closed set 
$H \subseteq \Sigma(\mathcal{H}) \cap \Gamma(\mathcal{H})$ depends on a given $\varepsilon>0$. We obtain

$$
\begin{aligned}
\frac{\sqrt{1-|\lambda|^{2}}|| k_{\lambda}||}{|g(\lambda)|} & \left(\left|\int_{\partial \mathbb{D}} P_{\lambda}(z) \bar{z} B(z) w_{g, h}(z) \frac{|d z|}{2 \pi}\right|-c(\lambda) \sqrt{1-|\lambda|^{2}}|| \frac{g}{1-\bar{\lambda} \zeta} \|\right) \\
& \leq \sqrt{\int_{\partial \mathbb{D}} P_{\lambda}(z) w_{h, h}(z) \frac{|d z|}{2 \pi}}+c(\lambda) .
\end{aligned}
$$

Taking nontangential limits gives

$$
\mathrm{nt}_{\lambda \rightarrow z} \frac{\sqrt{1-|\lambda|^{2}}|| k_{\lambda}||}{|g(\lambda)|}\left|w_{g, h}(z)\right| \leq \sqrt{w_{h, h}(z)}=\left|\frac{h}{g}(z)\right| \sqrt{w_{g, g}(z)}
$$

for a.e. $z \in H$. Since $\left|w_{g, h}(z)\right|=\left|\frac{h}{g}(z)\right| w_{g, g}(z) \neq 0$ we may divide and see that

$$
\operatorname{nt}_{\lambda \rightarrow z} \frac{\sqrt{1-|\lambda|^{2}}|| k_{\lambda}||}{|g(\lambda)|} \mid \leq\left(w_{g, g}(z)\right)^{-1 / 2}
$$

for a.e. $z \in H$. Since $\varepsilon>0$ was arbitrary it follows that the inequality is true for a.e. $z \in \Sigma(\mathcal{H}) \cap \Gamma(\mathcal{H})$. But the reverse inequality with an nt-liminf follows from Proposition 3.2. Thus we conclude that for every nonzero $g \in \mathcal{H}$ we have

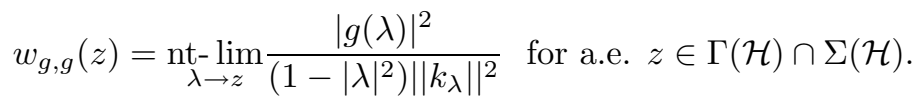

Of course this is trivial for $g=0$. Furthermore, on $\partial \mathbb{D} \backslash \Sigma(\mathcal{H})$ the function $w_{g, g}$ is 0 . Hence the more general statement of Theorem 5.2 now follows from Proposition 3.2 .

\section{ThE MAJORIZATION FUNCTION AND THE INDEX OF AN INVARIANT SUBSPACE}

In this section we shall prove Theorems 1.5, 1.7, 1.8, and some related results. We start out by explaining the nontangential boundary behaviour of the majorization function $k_{\mathcal{M}}(\lambda)$ of an invariant subspace $\mathcal{M}$ near the set $\Delta(\mathcal{H})$.

Lemma 6.1. Let $\mathcal{H} \neq(0)$ be a Hilbert space of analytic functions on $\mathbb{D}$ satisfying conditions (1.1) and (1.2).

(a) If $\mathcal{M} \in \operatorname{Lat}\left(M_{\zeta}, \mathcal{H}\right), \mathcal{M} \neq(0)$, then $\mathrm{nt}-\underline{\lim } k_{\mathcal{M}}(\lambda)>0$ for a.e. $z \in \Delta(\mathcal{H})$.

(b) There exists a nonzero $\mathcal{M} \in \operatorname{Lat}\left(M_{\zeta}, \stackrel{\lambda \rightarrow}{\mathcal{H})}\right.$ such that $\underset{\lambda \rightarrow z}{\operatorname{nt}} k_{\mathcal{M}}(\lambda)=0$ for a.e. $z \in \partial \mathbb{D} \backslash \Delta(\mathcal{H})$.

Furthermore, if $\mathcal{H}$ satisfies (1.1)-(1.4), then for every nonzero $\mathcal{M} \in \operatorname{Lat}\left(M_{\zeta}, \mathcal{H}\right)$ we have $\operatorname{nt}_{\lambda \rightarrow z} \lim _{\mathcal{M}}(\lambda)=1$ for a.e. $z \in \Sigma(\mathcal{H}) \cap \Gamma(\mathcal{H})=\Delta(\mathcal{H}) \cap \Gamma(\mathcal{H})$.

Proof. Let $k_{\lambda}(z)$ denote the reproducing kernel of $\mathcal{H}$, let $\mathcal{M} \in \operatorname{Lat}\left(M_{\zeta}, \mathcal{H}\right), \mathcal{M} \neq$ (0), let $0 \neq f \in \mathcal{M}$, and set $g=\frac{f}{1-\bar{\lambda} \zeta}$. Then $g \in \mathcal{M}$ and for each $\lambda \in \mathbb{D}$ we have

$$
1 \geq k_{\mathcal{M}}^{2}(\lambda) \geq \frac{|g(\lambda)|^{2}}{\|g\|^{2}\left\|k_{\lambda}\right\|^{2}}=\frac{1}{\left(1-|\lambda|^{2}\right) \|\left.\frac{f}{1-\bar{\lambda} \zeta}\right|^{2}} \frac{|f(\lambda)|^{2}}{\left(1-|\lambda|^{2}\right)|| k_{\lambda} \|^{2}} .
$$

By Lemma 2.2 and Proposition 3.5 we obtain

$$
\underset{\lambda \rightarrow-\varliminf_{\mathcal{L}}}{\lim } k_{\mathcal{M}}^{2}(\lambda) \geq \frac{\text { nt- } \lim _{\lambda \rightarrow z} \frac{|f(\lambda)|^{2}}{\left(1-|\lambda|^{2}\right) \mid k_{\lambda} \|^{2}}}{w_{f, f}(z)}>0
$$


for a.e. $z \in \Delta(\mathcal{H})$. This proves (a), and an application of Theorem 5.2 proves the statement of the last sentence of the lemma.

(b) We fix a nonzero function $f \in \mathcal{H}$, and let $\mathcal{N}=[f]$ be the closure of the polynomial multiples of $f$ in $\mathcal{H}$. Then $\mathcal{N}$ is an invariant subspace and it is easy to see that for every $g \in \mathcal{N}$ the meromorphic function $g / f$ has no poles in $\mathbb{D}$. Hence we can define a Hilbert space of analytic functions $\mathcal{H}_{1}$ by $\mathcal{H}_{1}=\{g / f: g \in$ $\mathcal{N}\},\|g / f\|_{1}=\|g\|$ for all $g \in \mathcal{N}$. It is clear that $\mathcal{H}_{1}$ satisfies conditions (1.1) and (1.2) and that $H^{\infty}$ is contained and dense in $\mathcal{H}_{1}$. Furthermore, one easily checks that $k_{\lambda}^{1}(z)=\frac{P_{\mathcal{N}} k_{\lambda}(z)}{f(\lambda) f(z)}$ is the reproducing kernel for $\mathcal{H}_{1}$. Hence we see that $\left.\left(1-|\lambda|^{2}\right)|| k_{\lambda}^{1}\right|^{2} \leq \frac{\left(1-|\lambda|^{2}\right)|| k_{\lambda} \|^{2}}{|f(\lambda)|^{2}}$ and so $\Delta(\mathcal{H}) \subseteq \Delta\left(\mathcal{H}_{1}\right)$.

By Theorem 4.1 (b) there is a sequence $\Lambda \subseteq \mathbb{D}$ that is interpolating for $\mathcal{H}_{1}$ and satisfies Ntl $\Lambda=\partial \mathbb{D} \backslash \Delta\left(\mathcal{H}_{1}\right)$ a.e. The interpolating property of $\Lambda$ implies that for fixed $\lambda_{0} \in \Lambda$ there is $h \in \mathcal{H}_{1}$ such that $h\left(\lambda_{0}\right)=1$ and $h(\lambda)=0$ whenever $\lambda \in \Lambda, \lambda \neq \lambda_{0}$. Then $\left(\zeta-\lambda_{0}\right) h$ is a nonzero function in $\mathcal{H}_{1}$ that is zero at all points in $\Lambda$. Hence $g=\left(\zeta-\lambda_{0}\right) h f \in \mathcal{H}$ is a nonzero function and it is zero at all points of $\Lambda$. Set $\mathcal{M}=[g] \subseteq \mathcal{N}$. Then it is clear that nt- $\underline{\lim } k_{\mathcal{M}}(\lambda)=0$ for a.e. $z \in \partial \mathbb{D} \backslash \Delta\left(\mathcal{H}_{1}\right)$.

Furthermore, for $\lambda \in \mathbb{D}$ we have

$$
k_{\mathcal{M}}^{2}(\lambda) \leq k_{\mathcal{N}}^{2}(\lambda)=\frac{\left\|P_{\mathcal{N}} k_{\lambda}\right\|^{2}}{\left\|k_{\lambda}\right\|^{2}}=\left(1-|\lambda|^{2}\right)\left\|k_{\lambda}^{1}\right\|^{2} \frac{|f(\lambda)|^{2}}{\left(1-|\lambda|^{2}\right)\left\|k_{\lambda}\right\|^{2}} .
$$

This implies that nt-lim$\underline{\lambda \rightarrow z}_{\mathcal{M}}(\lambda)=0$ for a.e. $z \in \Delta\left(\mathcal{H}_{1}\right) \cap(\partial \mathbb{D} \backslash \Delta(\mathcal{H}))$.

Proposition 6.2. Let $\mathcal{H}$ be a Hilbert space of analytic functions on $\mathbb{D}$ satisfying conditions (1.1)-(1.4), and let $\mathcal{M} \in \operatorname{Lat}\left(M_{\zeta}, \mathcal{H}\right)$ with ind $\mathcal{M}=1$.

If

$$
\left|\left\{z \in \partial \mathbb{D}: \underset{\lambda \rightarrow-\lim }{\operatorname{ng}} k_{\mathcal{M}}(\lambda)>0\right\} \cap \Gamma(\mathcal{H})\right|>0,
$$

then every $\mathcal{N} \in \operatorname{Lat}\left(M_{\zeta}, \mathcal{H}\right)$ with $\mathcal{M} \subseteq \mathcal{N}$ has index 1 .

Proof. A routine argument as in the beginning of the proof of Proposition 3.3 implies that there are constants $c>0$ and $0<R<1$ and a closed set $E \subseteq \partial \mathbb{D}$ such that $|E|>0$ and such that for all $\lambda \in \Omega_{E},|\lambda|>R$ we have

$$
k_{\mathcal{M}}(\lambda) \geq c \text { and }\left\|\frac{\zeta-\lambda}{1-\bar{\lambda} \zeta} f\right\| \geq c\|f\|
$$

for all $f \in \mathcal{H}$.

The remainder of the proof follows the general outline of the proof of Theorem 4.2 of ARS1. Let $g \in \mathcal{H}$. Then we must show that $\mathcal{M} \vee[g]$ has index 1 (see Theorem 3.13 of [Ri]). In fact, it suffices to show that for all nonzero $f \in \mathcal{M}$ there exists $\lambda \in \mathbb{D}$ with $f(\lambda) \neq 0$ and such that the function $\frac{g(z)-(g / f)(\lambda) f(z)}{z-\lambda} \in \mathcal{M} \vee[g]$. This was explained on p. 545 of $[\mathrm{AR}$, where one needs to recall that an invariant subspace has index one if and only if it has the division property. Let $h \in(\mathcal{M} \vee[g])^{\perp}$ and set

$$
H(\lambda)=\left\langle\frac{g-\frac{g}{f}(\lambda) f}{\zeta-\lambda}, h\right\rangle
$$


Then $H$ is a meromorphic function in $\mathbb{D}$, and we must show that $H$ is identically equal to zero.

Fix $\lambda \in \Omega_{E}$ such that $f(\lambda) \neq 0$, and set $\Phi=(1-\bar{\lambda} \zeta) P_{\mathcal{M}} k_{\lambda}$. Note that $f(\lambda) \neq 0$ implies that $\Phi(\lambda) \neq 0$. Thus, since $\mathcal{M}$ has index 1 , we have $\frac{f-\frac{f}{f}(\lambda) \Phi}{\zeta-\lambda} \in \mathcal{M}$. Of course we also have $\left\langle\frac{f-\frac{f}{\Phi}(\lambda) \Phi}{1-\bar{\lambda} \zeta}, h\right\rangle=0$. Hence we obtain

$$
\begin{aligned}
|H(\lambda)| & =\left|\left\langle\frac{g-\frac{g}{\Phi}(\lambda) \Phi}{\zeta-\lambda}, h\right\rangle\right| \\
& =\left|\left\langle\left(\frac{1}{\zeta-\lambda}+\frac{\bar{\lambda}}{1-\bar{\lambda} \zeta}\right)\left(g-\frac{g}{\Phi}(\lambda) \Phi\right), h\right\rangle\right| \\
& =\left(1-|\lambda|^{2}\right)\left|\left\langle\frac{g-\frac{g}{\Phi}(\lambda) \Phi}{(\zeta-\lambda)(1-\bar{\lambda} \zeta)}, h\right\rangle\right| \\
& \leq\left(1-|\lambda|^{2}\right)\left\|\frac{g-\frac{g}{\Phi}(\lambda) \Phi}{\zeta-\lambda}\right\|\left\|\left(I-\lambda \mathcal{M}_{\zeta}^{*}\right)^{-1} h\right\| \\
& \leq \frac{1}{c}\left(1-|\lambda|^{2}\right)|| \frac{g-\frac{g}{\Phi}(\lambda) \Phi}{1-\bar{\lambda} \zeta}\|\|\left(I-\lambda \mathcal{M}_{\zeta}^{*}\right)^{-1} h \| \quad \text { by }(6.1) \\
& \leq \frac{1}{c}\left(1-|\lambda|^{2}\right)\left(\left\|\frac{g}{1-\bar{\lambda} \zeta}\right\|+\left|\frac{g(\lambda)}{\Phi(\lambda)}\right|\left\|\frac{\Phi}{1-\bar{\lambda} \zeta}\right\|\right)\left\|\left(I-\lambda \mathcal{M}_{\zeta}^{*}\right)^{-1} h\right\| .
\end{aligned}
$$

By the fundamental inequality (1.5) we have $|g(\lambda)| \leq\left(1-|\lambda|^{2}\right)\left\|\frac{g}{1-\bar{\lambda} \zeta}\right\|\left\|k_{\lambda}\right\|$. Furthermore, $\Phi(\lambda)=\left(1-|\lambda|^{2}\right)\left\|P_{\mathcal{M}} k_{\lambda}\right\|^{2}$ and $\left\|\frac{\Phi}{1-\bar{\lambda} \zeta}\right\|=\left\|P_{\mathcal{M}} k_{\lambda}\right\|$. Hence it follows that $\left|\frac{g(\lambda)}{\Phi(\lambda)}\right|\left\|\frac{\Phi}{1-\bar{\lambda} \zeta}\right\| \leq \frac{1}{k_{\mathcal{M}}(\lambda)}\left\|\frac{g}{1-\bar{\lambda} \zeta}\right\|$.

Thus for $\lambda \in \Omega_{E},|\lambda|>R$ we have

$$
|H(\lambda)| \leq \frac{1}{c}\left(1+\frac{1}{c}\right)\left(1-|\lambda|^{2}\right)\left\|\frac{g}{1-\bar{\lambda} \zeta}\right\|\left\|\left(I-\lambda \mathcal{M}_{\zeta}^{*}\right)^{-1} h\right\|,
$$

and an application of Lemmas 2.2 and 3.1 implies that $|H(\lambda)| \rightarrow 0$ as $\lambda$ approaches a.e. $z \in E$ nontangentially. By Privalov's theorem this implies that $H$ is identically equal to 0 .

Theorem 6.3. If $\mathcal{H}$ satisfies (1.1)-(1.4), then

(a) if $|\Delta(\mathcal{H})|=0$, then there is an $\mathcal{M} \in \operatorname{Lat} M_{\zeta}$ with $\operatorname{ind} \mathcal{M}>1$,

(b) if $|\Delta(\mathcal{H}) \cap \Gamma(\mathcal{H})|>0$, then every nonzero $\mathcal{M} \in \operatorname{Lat} M_{\zeta}$ has index 1.

Proof. (a) Since $|\Delta(\mathcal{H})|=0$, Lemma 6.1 (b) implies the existence of a nonzero invariant subspace $\mathcal{M}$ such that $\underset{\lambda \rightarrow-\underline{\lim }}{\operatorname{lo}} k_{\mathcal{M}}(\lambda)=0$ for a.e. $z \in \partial \mathbb{D}$. For $\varepsilon>0$ set $\Lambda_{\varepsilon}=\left\{\lambda \in \mathbb{D}: k_{\mathcal{M}}(\lambda)<\varepsilon\right\}$. Then a.e. point of $\partial \mathbb{D}$ is a nontangential limit point of each $\Lambda_{\varepsilon}$; i.e., $\Lambda_{\varepsilon}$ is dominating for $\partial \mathbb{D}$. Hence Corollary 2.7 of ARS1 implies the existence of an invariant subspace $\mathcal{N} \supseteq \mathcal{M}$ with index $>1$.

(b) follows immediately from Lemma 6.1 (a) and Proposition 6.2.

For weighted Bergman spaces with so-called standard weights, $w(z)=\left(1-|z|^{2}\right)^{\alpha}$, $\alpha>-1$, the following theorem is equivalent to Theorem A of [ARS1]. It also implies Theorem 1.7 from our Introduction. Let $\mathcal{M}$ be an invariant subspace of $M_{\zeta}$ acting on $\mathcal{H}$. As in the previous proof for $0<\varepsilon \leq 1$ set $\Lambda_{\varepsilon}=\left\{\lambda \in \mathbb{D}: k_{\mathcal{M}}(\lambda)<\varepsilon\right\}$, and recall that a set $\Lambda \subseteq \mathbb{D}$ is called dominating for $\partial \mathbb{D}$ if Ntl $\Lambda=\partial \mathbb{D}$ a.e. 
Theorem 6.4. Let $\mathcal{H}$ be a Hilbert space of analytic functions satisfying conditions (1.1)-(1.4) and suppose that $\Gamma(\mathcal{H})$ has full measure in $\partial \mathbb{D}$. Let $\mathcal{M} \in \operatorname{Lat}\left(M_{\zeta}, \mathcal{H}\right)$ with ind $\mathcal{M}=1$.

Then the following are equivalent:

(a) for every $0<\varepsilon<1$ the set $\Lambda_{\varepsilon}$ is dominating for $\partial \mathbb{D}$,

(b) there is $0<\varepsilon<1$ such that $\Lambda_{\varepsilon}$ is dominating for $\partial \mathbb{D}$,

(c) there is an invariant subspace $\mathcal{N}$ of $M_{\zeta}$ such that $\mathcal{N} \supseteq \mathcal{M}$ and ind $\mathcal{N}>1$.

We mention that if $\mathcal{M}_{1} \subseteq \mathcal{M}_{2} \in \operatorname{Lat}\left(M_{\zeta}, \mathcal{H}\right)$ with ind $\mathcal{M}_{1}=$ ind $\mathcal{M}_{2}=1$, then $\frac{k_{\mathcal{M}_{1}}(\lambda)}{k_{\mathcal{M}_{2}}(\lambda)}=\frac{\left\|P_{\mathcal{M}_{1}} k_{\lambda}\right\|}{\left\|P_{\mathcal{M}_{2}} k_{\lambda}\right\|}$ and this equals the majorization function of $\mathcal{M}_{1}$ considered as a subspace of $\mathcal{H}=\mathcal{M}_{2}$. Thus the theorem automatically provides equivalent conditions for when there exists $\mathcal{N}$ with $\mathcal{M}_{1} \subseteq \mathcal{N} \subseteq \mathcal{M}_{2} \in \operatorname{Lat}\left(M_{\zeta}, \mathcal{H}\right)$ with ind $\mathcal{N}>1$. One just needs to replace $k_{\mathcal{M}}$ in the above theorem by $\frac{k_{\mathcal{M}_{1}}}{k_{\mathcal{M}_{2}}}$.

Proof. The implication (a) $\Longrightarrow$ (b) is obvious; (b) $\Longrightarrow$ (c) was done in ARS1, Corollary 2.7. In particular we note that the condition that $\Gamma(\mathcal{H})$ has full measure in $\partial \mathbb{D}$ is not needed for this implication. Finally, the contrapositive statement of (c) $\Longrightarrow$ (a) follows from Proposition 6.2.

In many instances there is a strong connection between the nontangential limiting behaviour of a function $f \in \mathcal{H}$ and the nontangential lim inf of $k_{[f]}(\lambda)$. For example, if $\varphi$ is an $L_{a}^{2}$-inner function, then the symmetric difference of the sets $\{z \in \partial \mathbb{D}$ :

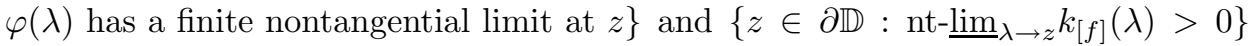
has measure 0 (see ARS1, Corollary 5.9). Another example is, if $f \in L_{a}^{2}$ is bounded in a set $\Omega_{E}$, where $E \subseteq \partial \mathbb{D}$ has finite entropy (i.e., the complementary intervals $\left\{I_{n}\right\}_{n}$ of $E$ satisfy $\left.\sum_{n}\left|I_{n}\right| \log \frac{1}{\left|I_{n}\right|}<\infty\right)$, then nt- $\underline{\lim }_{\lambda \rightarrow z} k_{[f]}(\lambda)>0$ for a.e. $z \in E$ (see ARS1, Theorem 5.11). Nevertheless it was also shown in [ARS1] that there are functions $f \in L_{a}^{2}$ which have finite nontangential limits a.e. on $\partial \mathbb{D}$ but such that nt- $\lim _{\lambda \rightarrow z} k_{[f]}(\lambda)=0$ for a.e. $z \in \partial \mathbb{D}$. It then follows from Theorem 6.4 (or Theorem A of [ARS1]) that there is a $g \in L_{a}^{2}$ such that ind $[f, g]=2$. The following theorem will imply that any such $g$ cannot have nontangential limits on any set of positive measure.

Theorem 6.5. Suppose $\mathcal{H}$ is a Hilbert space of analytic functions satisfying (1.1)(1.4).

If $f, g \in \mathcal{H}, f \neq 0$ such that $g / f$ has a nontangential limit on $F \subseteq \Gamma(\mathcal{H})$ with $|F|>0$, then ind $[f, g]=1$.

Proof. As in the proof of Proposition 6.2 we have to show that for some $\lambda \in \mathbb{D}$ the function $\frac{g(z)-(g / f)(\lambda) f(z)}{z-\lambda} \in[f] \vee[g]$. We let $h \in([f] \vee[g])^{\perp}$, and we shall show that the meromorphic function $H(\lambda)=\left\langle\frac{g-\frac{g}{f}(\lambda) f}{\zeta-\lambda}, h\right\rangle$ is identically equal to zero.

We note that the hypothesis implies via a routine argument that there are constants $c, C>0,0<R<1$, and a closed set $E \subseteq \partial \mathbb{D}$ such that $|E|>0$ and such that for all $\lambda \in \Omega_{E},|\lambda| \geq R$ we have $\left|\frac{g}{f}(\lambda)\right| \leq C$ and $\left\|\frac{\zeta-\lambda}{1-\bar{\lambda} \zeta} \varphi\right\| \geq c\|\varphi\|$ for all $\varphi \in \mathcal{H}$. 
As in the proof of Proposition 6.2 we obtain

$$
\begin{aligned}
|H(\lambda)| & =\left|\left\langle\frac{g-\frac{g}{f}(\lambda) f}{\zeta-\lambda}, h\right\rangle\right| \\
& =\left|\left\langle\left(\frac{1}{\zeta-\lambda}-\frac{1}{1-\bar{\lambda} \zeta}\right)\left(g-\frac{g}{f}(\lambda) f\right), h\right\rangle\right| \\
& =\left(1-|\lambda|^{2}\right)\left|\left\langle\frac{g-\frac{g}{f}(\lambda) f}{(\zeta-\lambda)(1-\bar{\lambda} \zeta)}, h\right\rangle\right| \\
& \leq\left(1-|\lambda|^{2}\right)\left\|\frac{g-\frac{g}{f}(\lambda) f}{\zeta-\lambda}\right\|\left\|\left(I-\lambda \mathcal{M}_{\zeta}^{*}\right)^{-1} h\right\| \\
& \leq \frac{1}{c}\left(1-|\lambda|^{2}\right)\left(\left\|\frac{g}{1-\bar{\lambda} \zeta}\right\|+C\left\|\frac{f}{1-\bar{\lambda} \zeta}\right\|\right)\left\|\left(I-\lambda \mathcal{M}_{\zeta}^{*}\right)^{-1} h\right\| .
\end{aligned}
$$

Thus as in the proof of Proposition 6.2, Lemmas 2.2 and 3.1 and an application of Privalov's theorem imply that $H=0$ in $\mathbb{D}$.

\section{Conditions on the ReProducing Kernel}

In this section we will discuss the results of this paper from the standpoint of the reproducing kernel. Recall from $[\mathrm{Ar}$ that a function $k: \mathbb{D} \times \mathbb{D} \rightarrow \mathbb{C}$ is called positive definite if $\sum_{i, j=1}^{n} a_{i} \bar{a}_{j} k\left(\lambda_{i}, \lambda_{j}\right) \geq 0$, whenever $n \geq 1, \lambda_{1}, \ldots, \lambda_{n} \in \mathbb{D}$ and $a_{1}, \ldots, a_{n} \in \mathbb{C}$. If $k(\lambda, z)$ is positive definite and analytic in the variable $z$ (and hence antianalytic in $\lambda$ ), then we will write $k_{\lambda}(z)=k(\lambda, z)$ and it is known that there is a unique Hilbert space $\mathcal{H}(k)$ of analytic functions on $\mathbb{D}$ with reproducing kernel $k$. Furthermore if $\left\{f_{n}\right\}_{n \geq 0}$ is any orthonormal basis for $\mathcal{H}(k)$, then $k_{\lambda}(z)=$ $\sum_{n \geq 0} \overline{f_{n}(\lambda)} f_{n}(z)$, where the sum converges uniformly on compact subsets of $\mathbb{D} \times$ $\mathbb{D}$ if $\mathcal{H}(k)$ is infinite dimensional. For a given reproducing kernel $k_{\lambda}(z)$ we set $l_{\lambda}(z)=(1-\bar{\lambda} z) k_{\lambda}(z)$. An easy and well-known argument shows that $M_{\zeta}$ defines a contraction operator on $\mathcal{H}(k)$ if and only if $l_{\lambda}(z)$ is positive definite. Thus a space $\mathcal{H}(k)$ satisfies conditions (1.1) and (1.2) if and only if $k$ is of the form $k_{\lambda}(z)=\frac{l_{\lambda}(z)}{1-\bar{\lambda} z}$ for some positive definite kernel $l_{\lambda}(z)$. It appears to be harder to determine precise conditions on $k$ that assure that $\mathcal{H}(k)$ satisfies all conditions (1.1)-(1.4). An easy case is when the defect operator $D=\left(I-M_{\zeta} M_{\zeta}^{*}\right)^{1 / 2}$ has finite rank. Note that $l_{\lambda}(z)=\left\langle D k_{\lambda}, D k_{z}\right\rangle$ for all $\lambda, z \in \mathbb{D}$. Thus $D$ has finite rank if and only if $l_{\lambda}(z)=$ $\sum_{n=0}^{N} \overline{\varphi_{n}(\lambda)} \varphi_{n}(z)$ for some $N \in \mathbb{N}$ and analytic functions $\varphi_{1}, \ldots, \varphi_{N}$.

We start with a well-known lemma.

Lemma 7.1. Suppose $\left\{\varphi_{n}\right\}_{n \geq 0}$ is a sequence of analytic functions on $\mathbb{D}$ such that $l_{\lambda}(z)=\sum_{n \geq 0} \overline{\varphi_{n}(\lambda)} \varphi_{n}(z)$ converges uniformly on compact subsets of $\mathbb{D} \times \mathbb{D}$.

If $k_{\lambda}(z)=\frac{l_{\lambda}(z)}{1-\bar{\lambda} z}$, then $f \in \mathcal{H}(k)$ if and only if $f=\sum_{n \geq 0} \varphi_{n} f_{n}$ for functions $f_{n} \in H^{2}$ with $\sum_{n \geq 0}\left\|f_{n}\right\|_{H^{2}}^{2}<\infty$. In fact we have

$$
\|f\|^{2}=\inf \left\{\sum_{n \geq 0}\left\|f_{n}\right\|_{H^{2}}^{2}: f_{n} \in H^{2}, f=\sum_{n \geq 0} \varphi_{n} f_{n}\right\}
$$

where the infimum is attained.

Proof. Define a linear map by $\left(T k_{\lambda}\right)(z)=\left\{\frac{\overline{\varphi_{n}(\lambda)}}{1-\bar{\lambda} z}\right\}_{n \geq 0}$. Then by checking on finite linear combinations of $k_{\lambda}, \lambda \in \mathbb{D}$, one proves that $T$ extends to define an 
isometric operator from $\mathcal{H}(k)$ into $\bigoplus_{n>0} H^{2}$. Also note that $T^{*}\left(\left\{f_{n}\right\}_{n \geq 0}\right)=$ $\sum_{n>0} \varphi_{n} f_{n}$. Thus whenever $\left\{f_{n}\right\}_{n \geq 0}$ is a sequence in $H^{2}$ with $\sum_{n>0}\left\|f_{n}\right\|_{H^{2}}^{2}<\infty$, then $\sum_{n \geq 0} \varphi_{n} f_{n}=T^{*}\left\{f_{n}\right\}_{n \geq 0} \in \mathcal{H}(k)$ and $\left\|\sum_{n \geq 0} \varphi_{n} f_{n}\right\|^{2}=\left\|T^{*}\left\{f_{n}\right\}_{n \geq 0}\right\|^{2} \leq$ $\sum_{n>0}\left\|f_{n}\right\|_{H^{2}}^{2}$. Furthermore if $f \in \mathcal{H}(k)$, then we set $\left\{f_{n}\right\}_{n \geq 0}=T f$ and note that $f=T^{*}(T f)=\sum_{n \geq 0} \varphi_{n} f_{n}$ and $\|f\|^{2}=\left\|T^{*}(T f)\right\|^{2}=\sum_{n \geq 0}\left\|f_{n}\right\|_{H^{2}}^{2}$.

Let $k_{\lambda}(z)$ be a reproducing kernel such that $\mathcal{H}(k)$ satisfies (1.1) and (1.2), and let $g$ be a meromorphic function on $\mathbb{D}$ such that $\mathcal{H}(k) / g \subseteq$ Hol $(\mathbb{D})$. Then $k_{\lambda}^{g}(z)=$ $\frac{k_{\lambda}(z)}{\overline{g(\lambda)} g(z)}$ defines the reproducing kernel for the space $\mathcal{H}\left(k^{g}\right)=\mathcal{H}(k) / g,\|f / g\|_{\mathcal{H}\left(k^{g}\right)}=$ $\|f\|_{\mathcal{H}(k)}$ for $f \in \mathcal{H}(k)$. We note that it is easy to see that $\mathcal{H}\left(k^{g}\right)$ satisfies (1.1) and (1.2), $M_{\zeta} \mid \mathcal{H}\left(k^{g}\right)$ is unitarily equivalent to $M_{\zeta} \mid \mathcal{H}(k), \Delta(\mathcal{H}(k))=\Delta\left(\mathcal{H}\left(k^{g}\right)\right)$, $\Sigma(\mathcal{H}(k))=\Sigma\left(\mathcal{H}\left(k^{g}\right)\right), \Gamma(\mathcal{H}(k))=\Gamma\left(\mathcal{H}\left(k^{g}\right)\right)$, and that $\mathcal{H}(k)$ satisfies (1.3) and (1.4) if and only if $\mathcal{H}\left(k^{g}\right)$ does.

In the following we will use $N(\mathbb{D})$ to denote the Nevanlinna class of the unit disc, i.e., the set of meromorphic functions that can be written as quotients of bounded analytic functions on $\mathbb{D}$.

Proposition 7.2. Let $\mathcal{H}(k)$ satisfy conditions (1.1) and (1.2) and let $f \in \mathcal{H}(k)$, $f \neq 0$. If the defect operator $D$ has finite rank, then $\mathcal{H}(k)$ satisfies conditions (1.1)-(1.4) if and only if $k_{\lambda} / f \in N(\mathbb{D})$ for every $\lambda \in \mathbb{D}$.

Furthermore, if $\mathcal{H}(k)$ satisfies conditions (1.1)-(1.4) and if the defect operator $D$ has finite rank, then $\Gamma(\mathcal{H}(k))$ has full measure in $\partial \mathbb{D}$ and $\Delta(\mathcal{H}(k))=\Sigma(\mathcal{H}(k))=\partial \mathbb{D}$ a.e.

Proof. Suppose first that $\mathcal{H}(k)$ satisfies conditions (1.1)-(1.4) and that $D$ has finite rank. As remarked before Lemma 7.1 we have $(1-\bar{\lambda} z) k_{\lambda}(z)=l_{\lambda}(z)=$ $\sum_{n=0}^{N} \overline{\varphi_{n}(\lambda)} \varphi_{n}(z)$ for some $N \in \mathbb{N}$ and analytic functions $\varphi_{0}, \cdots, \varphi_{N}$. In fact, by Lemma 7.1 each $\varphi_{i} \in \mathcal{H}(k)$. Fix $\alpha \in \mathbb{D}$ with $\varphi_{0}(\alpha) \neq 0$. It follows from conditions (1.3) and (1.4) that for each $n=1, \cdots, N$ the function $\frac{\varphi_{n}-\frac{\varphi_{n}}{\varphi_{0}}(\alpha) \varphi_{0}}{\zeta-\alpha} \in \mathcal{H}(k)$. Hence Lemma 7.1 implies that there are $f_{n, k} \in H^{2}$ such that

$$
\frac{\varphi_{n}-\frac{\varphi_{n}}{\varphi_{0}}(\alpha) \varphi_{0}}{\zeta-\alpha}=\sum_{k=0}^{N} \varphi_{k} f_{n, k} .
$$

This leads to the system of equations

$$
\sum_{k=1}^{N}\left(\delta_{n, k}-(\zeta-\alpha) f_{n, k}\right) \frac{\varphi_{k}}{\varphi_{0}}=\frac{\varphi_{n}}{\varphi_{0}}(\alpha)+(\zeta-\alpha) f_{n, 0}, \quad n=1, \cdots, N .
$$

Cramer's rule now easily implies that for each $n=1, \cdots, N$ we have $\frac{\varphi_{n}}{\varphi_{0}} \in N(\mathbb{D})$. Thus, if $f \in \mathcal{H}(k)$, then since $f / \varphi_{0}=\sum_{n=0}^{N} \frac{\varphi_{n}}{\varphi_{0}} f_{n}$ for functions $f_{n} \in H^{2}$ we conclude that $f / \varphi_{0} \in N(\mathbb{D})$. It follows that $k_{\lambda} / f$ is in the Nevanlinna class for every $\lambda \in \mathbb{D}$ and $f \in \mathcal{H}(k), f \neq 0$.

Now suppose that $f \in \mathcal{H}(k), f \neq 0$, that $D$ has finite rank, and that $k_{\lambda} / f \in$ $N(\mathbb{D})$ for every $\lambda \in \mathbb{D}$. Then $l_{\lambda} / f=(1-\bar{\lambda} \zeta) k_{\lambda} / f \in N(\mathbb{D})$ for every $\lambda \in \mathbb{D}$. Finite linear combinations of $l_{\lambda}, \lambda \in \mathbb{D}$, are dense in $\mathcal{H}(l)$; thus $\mathcal{H}(l)$ will have an orthonormal basis $\left\{\psi_{n}\right\}_{n \geq 0}$ such that each function $\psi_{n} / f \in N(\mathbb{D})$. By the hypothesis $\mathcal{H}(l)$ is finite dimensional; hence there is a nonzero Nevanlinna class function $h$ such that $\varphi_{n}=h \psi_{n} / f$ is in $H^{\infty}$ for each $n$. In fact we may arrange 
for an $h$ such that the $\varphi_{n}$ 's have no common zeros in the disc. According to the remark preceding Proposition 7.2 it suffices to prove that $\mathcal{H}\left(k^{g}\right)$ satisfies (1.3) and (1.4), where $k_{\lambda}^{g}(z)=\frac{\sum_{n=0}^{N} \overline{\varphi_{n}(\lambda)} \varphi_{n}(z)}{1-\bar{\lambda} z}, g=f / h$.

Since each $\varphi_{n}$ is bounded, we have $\sum_{n=0}^{N}\left\|\varphi_{n}\right\|_{\infty}^{2}=M^{2}<\infty$. For $f \in \mathcal{H}(k)$ choose $f_{0}, \ldots, f_{N} \in H^{2}$ such that $f=\sum_{n=0}^{N} \varphi_{n} f_{n}$ and $\|f\|^{2}=\sum_{n=0}^{N}\left\|f_{n}\right\|_{H^{2}}^{2}$. Then we see that it follows that $f \in H^{2}$ and $\|f\|_{H^{2}} \leq M\|f\|$. It also follows from Proposition 7.1 that $\left\|\varphi_{0} g\right\| \leq\|g\|_{H^{2}}$ for all $g \in H^{2}$.

Now let $\alpha \in \mathbb{D}$ be such that $\varphi_{0}(\alpha) \neq 0$ and write $b_{\alpha}(z)=\frac{\alpha-z}{1-\bar{\alpha} z}$. To verify both (1.3) and (1.4) it will be enough to show that $f / b_{\alpha} \in \mathcal{H}(k)$ whenever $f \in$ $\mathcal{H}(k)$ with $f(\alpha)=0$. In fact, it is easy to see that (1.3) follows and that dim $\mathcal{H}(k) \ominus(\zeta-\alpha) \mathcal{H}(k)=1$ for all such $\alpha$. If $\varphi_{0}(\alpha)=0$, then the choice of the $\varphi_{n}$ 's implies that there is some $n>0$ such that $\varphi_{n}(\alpha) \neq 0$, and the conclusion will follow by the same argument applied with $\varphi_{n}$ instead of $\varphi_{0}$.

Let $f \in \mathcal{H}(k)$ with $f(\alpha)=0$. Then

$$
\frac{f}{b_{\alpha}}=\frac{1-\frac{\varphi_{0}}{\varphi_{0}(\alpha)}}{b_{\alpha}} f+\frac{\varphi_{0}}{\varphi_{0}(\alpha)} \frac{f}{b_{\alpha}}
$$

and hence

$$
\begin{aligned}
\left\|\frac{f}{b_{\alpha}}\right\| & \leq\left\|\frac{1-\frac{\varphi_{0}}{\varphi_{0}(\alpha)}}{b_{\alpha}}\right\|_{\infty}\|f\|+\left\|\frac{\varphi_{0}}{\varphi_{0}(\alpha)} \frac{f}{b_{\alpha}}\right\| \\
& \leq\left\|1-\frac{\varphi_{0}}{\varphi_{0}(\alpha)}\right\|_{\infty}\|f\|+\frac{\left\|\frac{f}{b_{\alpha}}\right\|_{H^{2}}}{\left|\varphi_{0}(\alpha)\right|} \\
& \leq\left(1+\frac{\left\|\varphi_{0}\right\|_{\infty}}{\left|\varphi_{0}(\alpha)\right|}\right)\|f\|+\frac{M\|f\|}{\left|\varphi_{0}(\alpha)\right|} \leq\left(1+\frac{2 M}{\left|\varphi_{0}(\alpha)\right|}\right)\|f\| .
\end{aligned}
$$

Notice that this shows that $\Gamma(\mathcal{H}(k))$ contains the set of $z \in \partial \mathbb{D}$ where $\varphi_{0}(z) \neq$ 0 . Thus, $\Gamma(\mathcal{H}(k))$ has full measure in $\mathbb{D}$. Also note that for $\lambda \in \mathbb{D}$ we have $\left(1-|\lambda|^{2}\right) k_{\lambda}^{g}(\lambda)=\sum_{n=0}^{N}\left|\varphi_{n}(\lambda)\right|^{2} \leq M^{2}$ and hence $\Delta(\mathcal{H}(k))=\partial \mathbb{D}$. Of course, this implies $\Sigma(\mathcal{H}(k))=\partial \mathbb{D}$ as well.

Now we will consider reproducing kernels of the type

$$
k_{\lambda}(z)=\frac{\sum_{n=0}^{\infty} \overline{\varphi_{n}(\lambda)} \varphi_{n}(z)}{1-\bar{\lambda} z}, \quad \varphi_{n} / \varphi_{0} \in N(\mathbb{D}) \text { for each } n \geq 0 .
$$

It is clear that $\mathcal{H}(k)$ satisfies (1.1) and (1.2), and we will see shortly that (1.4) is satisfied as well. In fact, we have $\operatorname{dim} \mathcal{H}(k) \ominus(\zeta-\alpha) \mathcal{H}(k)=1$ for all $\alpha \in \mathbb{D}$. The following example shows that there are reproducing kernels $k$ of this type where $\mathcal{H}(k)$ does not satisfy condition (1.3). The corresponding operator $\left(M_{\zeta}, \mathcal{H}(k)\right)$ will be a weighted shift operator.

Example 7.3. We define a sequence of positive weights $\left\{w_{n}\right\}_{n \geq 0}$ by $w_{0}=1$, $w_{n}=1 / k$ ! if $n=k^{k}$ for some $k=1,2, \ldots$, and $w_{n}=w_{n-1}$ otherwise. Thus $w_{0}=\ldots=w_{3}=1, w_{4}=\ldots=w_{26}=1 / 2, w_{27}=\ldots=w_{255}=1 / 6$, etc. Set $f(z)=\sum_{n=0}^{\infty} \frac{z^{n}}{w_{n}}$. Then this power series defines an analytic function in $\mathbb{D}$, and a calculation shows that $(1-z) f(z)=1+\sum_{k=2}^{\infty}(k-1) !(k-1) z^{k^{k}}$. Hence $k_{\lambda}(z)=f(\bar{\lambda} z)$ defines a reproducing kernel of the type (7.1). 
For $g \in \mathcal{H}(k), g(z)=\sum_{n=0}^{\infty} \hat{g}(n) z^{n}$, we have $\|g\|^{2}=\sum_{n=0}^{\infty} w_{n}|\hat{g}(n)|^{2}$. This implies that with $g_{k}(z)=z^{k^{k}-1}$ we have $\left\|\zeta g_{k}\right\|^{2} /\left\|g_{k}\right\|^{2}=1 / k$; i.e., $M_{\zeta}$ is not bounded below. Thus, condition (1.3) cannot be satisfied for $\mathcal{H}(k)$. In fact, since $M_{\zeta}$ is a weighted shift operator, it follows that $M_{\zeta}-\lambda$ is not bounded below for any $\lambda \in \mathbb{D}$ (see $[\underline{S h}$, p. 69, Theorem 6).

If $k$ satisfies (7.1), then $\varphi_{n} / \varphi_{0}$ has nontangential limits a.e. on $\partial \mathbb{D}$, and by Fatou's lemma we see that for a.e. $z \in \partial \mathbb{D}$,

$$
\sum_{n \geq 0}\left|\frac{\varphi_{n}}{\varphi_{0}}(z)\right|^{2} \leq \underset{\lambda \rightarrow-\lim _{\lambda}}{ }\left(1-|\lambda|^{2}\right) \frac{\left\|k_{\lambda}\right\|^{2}}{\left|\varphi_{0}(\lambda)\right|^{2}} \leq \underset{\lambda t-\varlimsup_{\lambda \rightarrow z}}{ }\left(1-|\lambda|^{2}\right) \frac{\left\|k_{\lambda}\right\|^{2}}{\left|\varphi_{0}(\lambda)\right|^{2}} .
$$

Of course, by Proposition 3.5 and the remark following it we have equality on $\Delta(\mathcal{H}(k))$, i.e., on the set where all three quantities are finite.

Also recall from the remark after Question 1.2 that

$$
\left\{z \in \partial \mathbb{D}: \operatorname{nt}_{\lambda \rightarrow z} \varliminf_{i=}\left(1-|\lambda|^{2}\right) \frac{\left\|k_{\lambda}\right\|^{2}}{\left|\varphi_{0}(\lambda)\right|^{2}} \neq \underset{\lambda t-\varlimsup_{\lambda \rightarrow z}}{ }\left(1-|\lambda|^{2}\right) \frac{\left\|k_{\lambda}\right\|^{2}}{\left|\varphi_{0}(\lambda)\right|^{2}}\right\}
$$

is a.e. contained in $\Sigma(\mathcal{H}(k)) \backslash \Delta(\mathcal{H}(k))$.

Theorem 7.4. Suppose $k$ is of the form (7.1). Then

$$
\Sigma(\mathcal{H}(k)) \backslash \Delta(\mathcal{H}(k)) \subseteq\left\{z \in \partial \mathbb{D}: \sum_{n \geq 0}\left|\frac{\varphi_{n}}{\varphi_{0}}(z)\right|^{2} \neq \mathrm{nt}_{\lambda \rightarrow z}-\varlimsup_{i m}\left(1-|\lambda|^{2}\right) \frac{\left\|k_{\lambda}\right\|^{2}}{\left|\varphi_{0}(\lambda)\right|^{2}}\right\} \text { a.e. }
$$

and for every $g \in \mathcal{H}(k)$ we have

$$
w_{g, g}(z)=\mathrm{nt}_{\lambda \rightarrow z} \lim _{\lambda \rightarrow} \frac{|g(\lambda)|^{2}}{\left(1-|\lambda|^{2}\right)|| k_{\lambda}||^{2}} \text { for a.e. } z \in \Delta(\mathcal{H}(k)) .
$$

Proof. For $N \in \mathbb{N}$ let $k_{\lambda}^{N}(z)=\frac{\sum_{n=0}^{N} \overline{\varphi_{n}(\lambda)} \varphi_{n}(z)}{1-\bar{\lambda} z}$. Then by Lemma 7.1 the sequence of spaces $\mathcal{H}\left(k^{N}\right)$ is increasing in $N$ and for each $N \in \mathbb{N}, \mathcal{H}\left(k^{N}\right)$ is contractively contained in $\mathcal{H}(k)$; i.e., for each $g \in \mathcal{H}\left(k^{N}\right)$ we have $\|g\|_{\mathcal{H}(k)} \leq\|g\|_{\mathcal{H}\left(k^{N}\right)}$. Condition (7.1) and Proposition 7.2 imply that we can apply Theorem 5.2 with $\Gamma\left(\mathcal{H}\left(k^{N}\right)\right)=\Delta\left(\mathcal{H}\left(k^{N}\right)\right)=\partial \mathbb{D}$ a.e. Hence for every $g \in \mathcal{H}\left(k^{N}\right)$ the function $g / \varphi_{0}(\lambda)$ has nontangential limits $g / \varphi_{0}(z)$ a.e. on $\partial \mathbb{D}$ (in fact, $g / \varphi_{0} \in N(\mathbb{D})$ ), and

$$
\mathrm{nt}_{\lambda \rightarrow z} \lim _{\lambda \rightarrow}\left(1-|\lambda|^{2}\right)|| \frac{g}{1-\bar{\lambda} z} \|_{\mathcal{H}\left(k^{N}\right)}^{2}=\frac{\left|\frac{g}{\varphi_{0}}(z)\right|^{2}}{\sum_{n=0}^{N}\left|\frac{\varphi_{n}}{\varphi_{0}}(z)\right|^{2}} \text { for a.e. } z \in \partial \mathbb{D} \text {. }
$$

Thus by Lemma 2.2 we get for $g \in \mathcal{H}\left(k^{j}\right), j \leq N$ and a.e. $z \in \partial \mathbb{D}$,

$$
\begin{aligned}
w_{g, g}(z) & =\underset{\lambda \rightarrow z}{\operatorname{nt} \lim _{\rightarrow \rightarrow}}\left(1-|\lambda|^{2}\right)\left\|\frac{g}{1-\bar{\lambda} z}\right\|_{\mathcal{H}(k)}^{2} \\
& \leq \operatorname{nt}_{\lambda \rightarrow z} \lim _{\lambda \rightarrow}\left(1-|\lambda|^{2}\right)|| \frac{g}{1-\bar{\lambda} z} \|_{\mathcal{H}\left(k^{N}\right)}^{2} \\
& =\frac{\left|\frac{g}{\varphi_{0}}(z)\right|^{2}}{\sum_{n=0}^{N}\left|\frac{\varphi_{n}}{\varphi_{0}}(z)\right|^{2} .}
\end{aligned}
$$

This is valid for every $N \geq j$; hence for $g \in \mathcal{H}\left(k^{j}\right)$ we have

$$
w_{g, g}(z) \leq \frac{\left|\frac{g}{\varphi_{0}}(z)\right|^{2}}{\sum_{n=0}^{\infty}\left|\frac{\varphi_{n}}{\varphi_{0}}(z)\right|^{2}} \text { for a.e. } z \in \partial \mathbb{D} .
$$


This implies that for each such $g$ we have $w_{g, g}=0$ a.e. on the set $\{z \in \partial \mathbb{D}$ : $\left.\sum_{n \geq 0}\left|\frac{\varphi_{n}}{\varphi_{0}}(z)\right|^{2}=\infty\right\}$ a.e. or in other words $\left\{z \in \partial \mathbb{D}: w_{g, g}(z)>0\right\} \subseteq\{z \in \partial \mathbb{D}$ : $\left.\sum_{n \geq 0}\left|\frac{\varphi_{n}}{\varphi_{0}}(z)\right|^{2}<\infty\right\}$ a.e. Thus an application of Lemma 2.3 with the dense set $\mathcal{D}=\bigcup_{j \geq 0} \mathcal{H}\left(k^{j}\right)$ shows that $\Sigma(\mathcal{H}(k)) \subseteq\left\{z \in \partial \mathbb{D}: \sum_{n \geq 0}\left|\frac{\varphi_{n}}{\varphi_{0}}(z)\right|^{2}<\infty\right\}$ a.e.

Furthermore, inequality (7.3), along with Proposition 3.2 and the remark after Proposition 3.5, implies that for each $j \in \mathbb{N}$ and $g \in \mathcal{H}\left(k^{j}\right)$ equation (7.2) holds. Of course, this applies with the function $g=\varphi_{0}$. Thus taking nontangential limits in the fundamental inequality (1.5) we see that for all $f \in \mathcal{H}(k)$ we have

$$
\left|\frac{f}{\varphi_{0}}(z)\right|^{2} \leq \frac{w_{f, f}(z)}{w_{\varphi_{0}, \varphi_{0}}(z)} \text { for a.e. } z \in \Delta(\mathcal{H}(k)) .
$$

Now let $f \in \mathcal{H}(k)$. Then by Lemma 7.1 there is a sequence $\left\{g_{j}\right\} \rightarrow f$ and such that $g_{j} \in \mathcal{H}\left(k^{j}\right)$ for $j=1,2, \ldots$. Lemma 2.4 and the above inequality imply the existence of a subsequence $\left\{g_{j_{k}}\right\}$ such that $w_{g_{j_{k}}, g_{j_{k}}} \rightarrow w_{f, f}$ and $g_{j_{k}} / \varphi_{0} \rightarrow f / \varphi_{0}$ a.e. on $\Delta(\mathcal{H}(k))$. Thus, (7.2) holds for all $f \in \mathcal{H}(k)$.

We conclude this section by noting that a similar argument can be applied to other types of reproducing kernels. For example, let $u_{\lambda}(z)$ be positive definite and sesquianalytic with $u_{\lambda}(\lambda)<1$ for all $\lambda \in \mathbb{D}$, and let $k_{\lambda}(z)=\frac{1}{1-\lambda z} \frac{1}{1-u_{\lambda}(z)}$. Then it follows that there are $H^{\infty}$-functions $u_{n}$ such that $u_{\lambda}(z)=\sum_{n>1} \overline{u_{n}(\lambda)} u_{n}(z)$. Thus by a version of Fatou's Theorem (see p.81 of [RR] it follows that for a.e. $z \in \partial \mathbb{D}$ we have $u_{z}(z):=\sum_{n \geq 1}\left|u_{n}(z)\right|^{2}=\underset{\lambda \rightarrow z}{\operatorname{nt}} \lim _{\lambda}(\lambda) \leq 1$. In this case one shows $(7.2)$ and that $\Delta(\mathcal{H}(k))=\Sigma(\mathcal{H}(k))=\left\{z \stackrel{\lambda \rightarrow z}{\in} \dot{D} \mathbb{D}: u_{z}(z)<1\right\}$. For the proof one uses an approximation of $k$ with kernels $k_{\lambda}^{N_{1}, N_{2}}(z)=\frac{\sum_{k=0}^{N_{1}}\left(\sum_{n=1}^{N_{2}} \overline{u_{n}(\lambda)} u_{n}(z)\right)^{k}}{1-\bar{\lambda} z}$. We omit the details.

\section{EXAMPLES}

We will now present examples of spaces $\mathcal{H}$ that satisfy conditions (1.1)-(1.4), have a reproducing kernel of the type as in $(7.1)$, but with $|\Delta(\mathcal{H})|=0$ and $\Sigma(\mathcal{H})=\partial \mathbb{D}$. Thus from Lemma 2.3 it is clear that all of these spaces $\mathcal{H}$ contain functions $f$ such that $w_{f, f} \neq 0$ or in other words such that $\left\|\zeta^{n} f\right\| \nrightarrow 0$ as $n \rightarrow \infty$.

We will further see that for some of those spaces one has $\left\|\zeta^{n} f\right\| \nrightarrow 0$ whenever $f \neq 0$, i.e. conditions (b) and (c) of Corollary 1.6 are not equivalent in the absence of $\Sigma(\mathcal{H}) \subseteq \Gamma(\mathcal{H})$, while for other spaces $\left\|\zeta^{n} f\right\| \rightarrow 0$ for some nonzero $f \in \mathcal{H}$, and so (c) and (d) of Corollary 1.6 are not equivalent either without the assumption $\Sigma(\mathcal{H}) \subseteq \Gamma(\mathcal{H})$.

Example 8.1 Let $\Lambda=\left\{\lambda_{n}\right\}_{n \geq 0}$ be a sequence of distinct points in $\mathbb{D}$ such that $\Lambda$ has no accumulation point in $\mathbb{D}$, let $f$ be an analytic function in $\mathbb{D}$ with simple zeros at $\lambda_{n}$ and no other zeros, and let $c, w_{n}>0$ be such that $\sum_{n=0}^{\infty} \frac{w_{n}}{1-\left|\lambda_{n}\right|^{2}} \leq c$.

Note that for all $a_{n} \in \mathbb{C}$,

$$
\frac{1}{c}\left(\sum_{n=0}^{\infty}\left|a_{n}\right|\right)^{2} \leq \sum_{n=0}^{\infty}\left(1-\left|\lambda_{n}\right|^{2}\right) \frac{\left|a_{n}\right|^{2}}{w_{n}} \leq \sum_{n=0}^{\infty} \frac{\left|a_{n}\right|^{2}}{w_{n}} .
$$

Thus whenever $\sum_{n=0}^{\infty} \frac{\left|a_{n}\right|^{2}}{w_{n}}<\infty, \sum_{n=0}^{\infty} \frac{a_{n}}{\zeta-\lambda_{n}}$ defines a meromorphic function in $\mathbb{D}$ with a simple pole at each $\lambda_{n}$. We define $\mathcal{H}$ to be the set of analytic functions $g$ of 
the form $g=f\left(u+\sum_{n=0}^{\infty} \frac{a_{n}}{\zeta-\lambda_{n}}\right)$, where $u \in H^{2}$ and $\sum_{n=0}^{\infty} \frac{\left|a_{n}\right|^{2}}{w_{n}}<\infty$. Note that if $g \in \mathcal{H}$, then the function $u$ and the coefficients $\left\{a_{n}\right\}_{n=0}^{\infty}$ are uniquely determined. Thus we may define a Hilbert space norm on $\mathcal{H}$ by setting

$$
\|g\|^{2}=\left\|f\left(u+\sum_{n=0}^{\infty} \frac{a_{n}}{\zeta-\lambda_{n}}\right)\right\|^{2}=\frac{1}{c}\|u\|_{H^{2}}^{2}+\sum_{n=0}^{\infty} \frac{\left|a_{n}\right|^{2}}{w_{n}}
$$

We verify that $\mathcal{H}$ is a space of analytic functions that satisfies conditions (1.1)(1.4). In fact, an easy computation shows that the reproducing kernel is

$$
k_{\lambda}(z)=\overline{f(\lambda)} f(z)\left(\frac{c}{1-\bar{\lambda} z}+\sum_{n=0}^{\infty} \frac{w_{n}}{\left(z-\lambda_{n}\right)\left(\bar{\lambda}-\bar{\lambda}_{n}\right)}\right)
$$

whenever $\lambda \notin \Lambda$, and $k_{\lambda_{n}}(z)=f(z) \overline{f^{\prime}\left(\lambda_{n}\right)} \frac{w_{n}}{z-\lambda_{n}}$ for each $n$. Furthermore, if $g=$ $f\left(u+\sum_{n=0}^{\infty} \frac{a_{n}}{\zeta-\lambda_{n}}\right) \in \mathcal{H}$, then $\zeta g=f\left(\zeta u+\sum_{n=0}^{\infty} a_{n}+\sum_{n=0}^{\infty} \frac{\lambda_{n} a_{n}}{\zeta-\lambda_{n}}\right)$, so by inequality (8.1) we have

$$
\|\zeta g\|^{2}=\frac{\|u\|_{H^{2}}^{2}+\left|\sum_{n=0}^{\infty} a_{n}\right|^{2}}{c}+\sum_{n=0}^{\infty}\left|\lambda_{n}\right|^{2} \frac{\left|a_{n}\right|^{2}}{w_{n}} \leq\|g\|^{2} .
$$

Thus, $\mathcal{H}$ satisfies conditions (1.1) and (1.2). In order to verify (1.3) and (1.4) it suffices to prove that whenever $\lambda \in \mathbb{D}$ and $g \in \mathcal{H}$ with $g(\lambda)=0$, then $g /(\zeta-\lambda) \in \mathcal{H}$ (see Lemma 2.1 of [Ri] $)$. Let $g=f\left(u+\sum_{n=0}^{\infty} \frac{a_{n}}{\zeta-\lambda_{n}}\right)$ with $g(\lambda)=0$. We first assume $\lambda \neq \lambda_{n}$ for all $n$. Then $u(\lambda)+\sum_{n=0}^{\infty} \frac{a_{n}}{\lambda-\lambda_{n}}=0$, so

$$
\frac{g(z)}{z-\lambda}=f(z)\left(\frac{u(z)-u(\lambda)}{z-\lambda}+\sum_{n=0}^{\infty} \frac{a_{n}}{z-\lambda_{n}} \frac{1}{\lambda_{n}-\lambda}\right) \in \mathcal{H} .
$$

If $\lambda=\lambda_{k}$ for some $k$, then $a_{k}=0$. In this case,

$$
\frac{g(z)}{z-\lambda_{k}}=f(z)\left(\frac{u(z)-u\left(\lambda_{k}\right)}{z-\lambda_{k}}+\frac{u\left(\lambda_{k}\right)+\sum_{n=0}^{\infty} \frac{a_{n}}{\lambda_{k}-\lambda_{n}}}{z-\lambda_{k}}+\sum_{n=0, n \neq k}^{\infty} \frac{a_{n}}{z-\lambda_{n}} \frac{1}{\lambda_{n}-\lambda_{k}}\right)
$$

and this function is in $\mathcal{H}$ also.

Next we note that the reproducing kernel for $\mathcal{H}$ can be written as $k_{\lambda}(z)=$ $f(z) \overline{f(\lambda)} \frac{l_{\lambda}(z)}{1-\bar{\lambda} z}$, where

$$
l_{\lambda}(z)=c-\sum_{n=0}^{\infty} \frac{w_{n}}{1-\left|\lambda_{n}\right|^{2}}+\sum_{n=0}^{\infty} \frac{w_{n}}{1-\left|\lambda_{n}\right|^{2}} \psi_{n}(z) \overline{\psi_{n}(\lambda)}
$$

and $\psi_{n}(z)=\frac{1-\overline{\lambda_{n}} z}{\lambda_{n}-z}$. Thus, $k_{\lambda}(z)$ is of the type (7.1).

The function $f$ is in $\mathcal{H}$ and $\left(1-|\lambda|^{2}\right) \frac{\left\|k_{\lambda}\right\|^{2}}{|f(\lambda)|^{2}}=l_{\lambda}(\lambda)$; thus the nontangential cluster set of $\Lambda$ is contained in $\partial \mathbb{D} \backslash \Delta(\mathcal{H})$. In particular, if the set $\Lambda$ is dominating for $\partial \mathbb{D}$, then $|\Delta(\mathcal{H})|=0$. On the other hand, $\left(1-|\lambda|^{2}\right)\left\|\frac{f}{1-\bar{\lambda} \zeta}\right\|^{2}=1$ for all $\lambda \in \mathbb{D}$; hence $w_{f, f}=1$ and so $\Sigma(\mathcal{H})=\partial \mathbb{D}$.

We will now specify choices of $\Lambda,\left\{w_{n}\right\}_{n \geq 0}$ and $c$ as above such that $|\Delta(\mathcal{H})|=0$, $\Sigma(\mathcal{H})=\partial \mathbb{D}$, and such that $\left\|\zeta^{n} g\right\| \nrightarrow 0$ for all $g \in \mathcal{H}, g \neq 0$. For this we choose $\Lambda$ to be dominating for $H^{\infty}$ and interpolating for the Bergman space $L_{a}^{2}$ (see Section 4 for a definition of an interpolating sequence). It is known that such sequences exist 
(see e.g. [Se, [DS]). Next we choose $\left\{w_{n}\right\}_{n \geq 0}$ and $c>0$ such that $\sum_{n=0}^{\infty} \frac{w_{n}}{1-\left|\lambda_{n}\right|^{2}} \leq c$ and $w_{n} \leq\left(1-\left|\lambda_{n}\right|^{2}\right)^{2}$ for all $n$.

If $g=f\left(u+\sum_{n=0}^{\infty} \frac{a_{n}}{\zeta-\lambda_{n}}\right) \in \mathcal{H}$ such that $\left\|\zeta^{k} g\right\| \rightarrow 0$ as $k \rightarrow \infty$, then $u=0$ and $\sum_{n=0}^{\infty} a_{n} \lambda_{n}^{k}=0$ for all $k \geq 0$. Thus $\sum_{n=0}^{\infty} a_{n} p\left(\lambda_{n}\right)=0$ for every polynomial $p$. Furthermore, for any $f \in L_{a}^{2}$ we have

$$
\begin{aligned}
\left(\sum_{n=0}^{\infty}\left|a_{n} f\left(\lambda_{n}\right)\right|\right)^{2} & \leq \sum_{n=0}^{\infty} \frac{\left|a_{n}\right|^{2}}{w_{n}} \sum_{n=0}^{\infty} w_{n}\left|f\left(\lambda_{n}\right)\right|^{2} \\
& \leq\|g\|^{2} \sum_{n=0}^{\infty}\left(1-\left|\lambda_{n}\right|^{2}\right)^{2}\left|f\left(\lambda_{n}\right)\right|^{2} \leq M\|g\|^{2}\|f\|_{L_{a}^{2}}^{2}
\end{aligned}
$$

where the last inequality follows from (4.1) since the reproducing kernel for $L_{a}^{2}$ is $k_{\lambda}(z)=(1-\bar{\lambda} z)^{-2}$. It follows that $\sum_{n=0}^{\infty} a_{n} f\left(\lambda_{n}\right)=0$ for every $f \in L_{a}^{2}$. Since $\Lambda=\left\{\lambda_{n}\right\}_{n \geq 0}$ is interpolating for $L_{a}^{2}$ for each $n$, we may choose $f_{n} \in L_{a}^{2}$ such that $f_{n}\left(\lambda_{n}\right) \neq 0$ but $f_{n}\left(\lambda_{j}\right)=0$ for all $j \neq n$. Hence $a_{n}=0$ for all $n$, i.e. $g=0$.

Next we will describe a situation where $|\Delta(\mathcal{H})|=0, \Sigma(\mathcal{H})=\partial \mathbb{D}$, but $\| \zeta^{n} g|| \rightarrow 0$ for some $g \in \mathcal{H}, g \neq 0$. For the construction we set

$$
\mathcal{B}=\left\{f \in \operatorname{Hol}(\mathbb{D}): \sup _{z \in \mathbb{D}} \sqrt{1-|z|^{2}}|f(z)|<\infty\right\},\|f\|_{\mathcal{B}}=\sup _{z \in \mathbb{D}} \sqrt{1-|z|^{2}}|f(z)| .
$$

By Theorem 2 of [RS], there is a sequence $\Lambda=\left\{\lambda_{n}\right\}_{n \geq 0}$ with no limit point in $\mathbb{D}$ and such that for all $f \in \mathcal{B}$,

$$
\sup _{n \geq 0} \sqrt{1-\left|\lambda_{n}\right|^{2}}\left|f\left(\lambda_{n}\right)\right| \geq 1 / 2|| f \|_{\mathcal{B}} .
$$

We may also assume that $\Lambda$ is dominating for $H^{\infty}$. The linear operator $T: \mathcal{B} \rightarrow l^{\infty}$, $T f=\left\{\sqrt{1-\left|\lambda_{n}\right|^{2}} f\left(\lambda_{n}\right)\right\}_{n \geq 0}$ is bounded and bounded from below. Furthermore it is clear that $T$ cannot be onto, because if $\{1,0,0, \ldots\}=T f$ for some $f \in \mathcal{B}$, then the function $\left(\zeta-\lambda_{0}\right) f \in \mathcal{B}$ is zero at all points $\lambda_{n}$; hence $\left\|\left(\zeta-\lambda_{0}\right) f\right\|_{\mathcal{B}}=0$. This would imply $f=0$, which would be a contradiction. Finally, we claim that the range of $T$ is weak ${ }^{*}$ closed in $l^{\infty}$. This is a standard argument, which we indicate for completeness. Since $l^{\infty}$ is the dual of the separable space $l^{1}$ it follows from the Krein-Smulian Theorem that it is enough to check that the range of $T$ is weak* sequentially closed. If $\left\{T f_{k}\right\}$ is a weak* convergent sequence, then it must be norm bounded. Since $T$ is bounded below, the sequence $\left\{f_{k}\right\}$ is norm bounded in $\mathcal{B}$. The proof can now be completed with a normal families argument.

It follows that there is a nonzero element $\left\{a_{n}\right\}_{n \geq 0} \in l^{1}$ such that

$$
\sum_{n \geq 0} a_{n} \sqrt{1-\left|\lambda_{n}\right|^{2}} f\left(\lambda_{n}\right)=0 \text { for every } f \in \mathcal{B} \text {. }
$$

We now set $w_{n}=\left|a_{n}\right|\left(1-\left|\lambda_{n}\right|^{2}\right)$ whenever $a_{n} \neq 0$. Then $\sum_{\left\{n: a_{n} \neq 0\right\}} \frac{w_{n}}{1-\left|\lambda_{n}\right|^{2}}=$ $\sum_{n \geq 0}\left|a_{n}\right|<\infty$; thus we may choose $\left\{w_{n}\right\}$ and $c$ such that $w_{n}>0$ for all $n$, $\sum_{n \geq 0} \frac{w_{n}}{1-\left|\lambda_{n}\right|^{2}} \leq c$ and $w_{n}=\left|a_{n}\right|\left(1-\left|\lambda_{n}\right|^{2}\right)$ whenever $a_{n} \neq 0$.

We set $g=f \sum_{n=0}^{\infty} \frac{a_{n} \sqrt{1-\left|\lambda_{n}\right|^{2}}}{z-\lambda_{n}}$. Then $\sum_{n=0}^{\infty} \frac{\left|a_{n}\right|^{2}\left(1-\left|\lambda_{n}\right|^{2}\right)}{w_{n}}=\sum_{n \geq 0}\left|a_{n}\right|<\infty$, so $g \in \mathcal{H}$. Furthermore, since $\sum_{n \geq 0} a_{n} \sqrt{1-\left|\lambda_{n}\right|^{2}} \lambda_{n}^{j}=0$ for each $j \geq 0$ we see that $\zeta^{k} g=f \sum_{n=0}^{\infty} \frac{a_{n} \lambda_{n}^{k} \sqrt{1-\left|\lambda_{n}\right|^{2}}}{z-\lambda_{n}}$ and $\left\|\zeta^{k} g\right\|^{2}=\sum_{n=0}^{\infty} \frac{\left|a_{n}\right|^{2}\left|\lambda_{n}\right|^{2 k}\left(1-\left|\lambda_{n}\right|^{2}\right)}{w_{n}}$. By the dominated convergence theorem this converges to zero as $k \rightarrow \infty$. 


\section{Further COMMENTS AND A QUESTION}

All of our main theorems of Sections 5 and 6 had the set $\Gamma(\mathcal{H})$ in the hypothesis, and often we assumed that $\Sigma(\mathcal{H}) \subseteq \Gamma(\mathcal{H})$ a.e. We mentioned in the Introduction that an answer to Question 1.2 is available for analytic $P^{2}(\mu)$-spaces and it does not require that one knows beforehand that $\Sigma\left(P^{2}(\mu)\right) \subseteq \Gamma\left(P^{2}(\mu)\right)$ a.e. (see ARS2]). We will now show that $\Delta\left(P^{2}(\mu)\right) \subseteq \Gamma\left(P^{2}(\mu)\right)$ a.e.

The following lemma will be needed. It can also be used to see that the Bergman spaces with standard weights $\left(1-|z|^{2}\right)^{\gamma}, \gamma>-1$ all satisfy the hypothesis (1.8). Of course, that is a fairly simple matter anyway, but the lemma applies to other measures as well. For $\lambda \in \mathbb{D}, 0<r<1$ we define $D_{\lambda, r}=\left\{w \in \mathbb{D}:\left|\frac{w-\lambda}{1-\bar{\lambda} w}\right|<r\right\}$.

Lemma 9.1. Let $\mu$ be a measure in $\overline{\mathbb{D}}$ such that $P^{2}(\mu)$ satisfies (1.1)-(1.4). Write $k_{\lambda}(z)$ for the reproducing kernel of $P^{2}(\mu)$, let $\lambda \in \mathbb{D}$ and $0<r<1$, and set

$$
K(\lambda)=\mu\left(D_{\lambda, r}\right) \sup _{w \in D_{\lambda, r}}\left\|k_{w}\right\|^{2} .
$$

Then $\|f\|^{2} \leq \frac{1+K(\lambda)}{r^{2}}\left\|\frac{\zeta-\lambda}{1-\bar{\lambda} \zeta} f\right\|^{2}$ for all $f \in P^{2}(\mu)$.

Recall the definition of the quantity $c_{\mathcal{H}}(\lambda)$ that was used in (1.9) for the definition of the set $\Gamma(\mathcal{H})$. The lemma implies that $1 \geq c_{P^{2}(\mu)}(\lambda) \geq \frac{r}{\sqrt{1+K(\lambda)}}$.

Proof. For $z, \lambda \in \mathbb{D}$ set $b_{\lambda}(z)=\frac{z-\lambda}{1-\bar{\lambda} z}$. Since $\left|b_{\lambda}\right| \geq r$ in $\overline{\mathbb{D}} \backslash D_{\lambda, r}$, it suffices to show that for all $f \in P^{2}(\mu)$ we have $\int_{D_{\lambda, r}}|f|^{2} d \mu \leq \frac{K(\lambda)}{r^{2}}\left\|b_{\lambda} f\right\|^{2}$.

To see this let $f \in P^{2}(\mu)$. Then by the maximum principle,

$$
\begin{aligned}
\int_{D_{\lambda, r}}|f|^{2} d \mu & \leq \sup _{w \in \partial D_{\lambda, r}}|f(w)|^{2} \mu\left(D_{\lambda, r}\right) \leq \frac{1}{r^{2}} \sup _{w \in \partial D_{\lambda, r}}\left|b_{\lambda}(w) f(w)\right|^{2} \mu\left(D_{\lambda, r}\right) \\
& \leq \frac{1}{r^{2}}\left\|b_{\lambda} f\right\|^{2} \sup _{w \in \partial D_{\lambda, r}}\left\|k_{w}\right\|^{2} \mu\left(D_{\lambda, r}\right) \leq \frac{K(\lambda)}{r^{2}}\left\|b_{\lambda} f\right\|^{2} .
\end{aligned}
$$

Theorem 9.2. Let $\mu$ be a measure in $\overline{\mathbb{D}}$ such that $P^{2}(\mu)$ satisfies (1.1)-(1.4).

Then nt- $\lim _{\lambda \rightarrow z} c_{P^{2}(\mu)}(\lambda)=1$ for a.e. $z \in \Delta\left(P^{2}(\mu)\right)$. Thus,

$$
\Delta\left(P^{2}(\mu)\right) \subseteq \Gamma\left(P^{2}(\mu)\right) \text { a.e. }
$$

By use of the results of ARS2 this implies that $\Sigma\left(P^{2}(\mu)\right) \subseteq \Gamma\left(P^{2}(\mu)\right)$ a.e.

Proof. We fix $0<r<1$. According to Lemma 9.1 it suffices to prove that for a.e. $z \in \Delta\left(P^{2}(\mu)\right), K(\lambda) \rightarrow 0$ as $\lambda$ approaches $z$ nontangentially.

For each $\lambda \in \mathbb{D}$ we have

$$
\frac{\mu\left(D_{\lambda, r}\right)}{1-|\lambda|^{2}} \leq \frac{1-|\lambda|^{2}}{(1-r)^{2}} \int_{D_{\lambda, r}}|1-\bar{\lambda} w|^{-2} d \mu(w) \leq \frac{1-|\lambda|^{2}}{(1-r)^{2}} \int_{\mathbb{D}}|1-\bar{\lambda} w|^{-2} d \mu(w) .
$$

Hence it follows from Lemma 2.2 that $\frac{\mu\left(D_{\lambda, r}\right)}{1-|\lambda|^{2}} \rightarrow 0$ as $\lambda$ approaches a.e. $z \in \partial \mathbb{D}$.

Hence

$K(\lambda)=\frac{\mu\left(D_{\lambda, r}\right)}{1-|\lambda|^{2}} \sup _{w \in D_{\lambda, r}}\left(1-|w|^{2}\right)\left\|k_{w}\right\|^{2} \frac{1-|\lambda|^{2}}{1-|w|^{2}} \leq \frac{\mu\left(D_{\lambda, r}\right)}{1-|\lambda|^{2}} \sup _{w \in D_{\lambda, r}}\left(1-|w|^{2}\right)\left\|k_{w}\right\|^{2} C$

for some $C>0$ that depends only on $r$. The conclusion now follows from the definition of $\Delta\left(P^{2}(\mu)\right)$ (take $\left.g=1\right)$. 
This raises the following question.

Question 9.3. Suppose $\mathcal{H}$ satisfies conditions (1.1)-(1.4). Is it true that

$$
\Delta(\mathcal{H}) \subseteq \Gamma(\mathcal{H}) ?
$$

If this were true, then Theorem 6.3 would imply that conditions (a) and (b) of Corollary 1.6 are equivalent without the extra hypothesis that $\Sigma(\mathcal{H}) \subseteq \Gamma(\mathcal{H})$.

Proposition 9.4. Let $\mathcal{H}$ be a space of analytic functions on $\mathbb{D}$ that satisfies conditions (1.1)-(1.4). Suppose that $|\Sigma(\mathcal{H})|=0$.

If $f \in \mathcal{H}$ is contained in an invariant subspace of index $>1$, then for any $n \geq 1$ or $n=\infty, f$, is contained in an invariant subspace of index $n$.

Proof. The hypothesis implies that $\left\|M_{\zeta}^{n} f\right\| \rightarrow 0$ and $\left\|M_{\zeta}^{* n} f\right\| \rightarrow 0$ for every $f \in \mathcal{H}$; i.e., the operator $M_{\zeta} \in C_{00}$. If $\mathcal{M}$ is any invariant subspace of $M_{\zeta}$, then the compressed operator $T=P_{\mathcal{M}^{\perp}} M_{\zeta} \mid \mathcal{M}^{\perp}$ satisfies $T^{n}=P_{\mathcal{M}^{\perp}} M_{\zeta}^{n} \mid \mathcal{M}^{\perp}$ and $T^{* n}=$ $M_{\zeta}^{* n} \mid \mathcal{M}^{\perp}$ for all $n \geq 0$; thus $T \in C_{00}$. If $f \in \mathcal{H}$ is contained in an invariant subspace of index $>1$, then we set $\mathcal{M}=[f]$ and conclude from Theorem 2.6 of ARS1 that $T=P_{\mathcal{M}^{\perp}} M_{\zeta} \mid \mathcal{M}^{\perp} \in \mathbb{A}$; i.e., $\left\|M_{\varphi}^{*} \mid \mathcal{M}^{\perp}\right\|=\|\varphi\|_{\infty}$ for every $\varphi \in H^{\infty}$. Theorems of $\mathrm{Be}$ and $\mathrm{ABFP}$ say that $\mathbb{A} \cap C_{00}=\mathbb{A}_{\aleph_{0}} \cap C_{00}$. That means that there are sequences of functions $\left\{f_{i}\right\}_{i=1}^{\infty}$ and $\left\{g_{j}\right\}_{j=1}^{\infty}$ in $\mathcal{M}^{\perp}$ such that $\left\langle h f_{i}, g_{j}\right\rangle=h(0) \delta_{i j}$ for each $h \in H^{\infty}$.

For $n \geq 1$ we set $\mathcal{N}_{n}=[f] \vee \bigvee_{i=1}^{n-1}\left[f_{i}\right]$. Similarly, if $n=\infty$, we set $\mathcal{N}_{n}=$ $[f] \vee \bigvee_{i=1}^{\infty}\left[f_{i}\right]$. It is easy to check that $\mathcal{N}_{n}$ has index $n$.

\section{REFERENCES}

[AR] Aleman, Alexandru, and Richter, Stefan, Some sufficient conditions for the division property of invariant subspaces in weighted Bergman spaces. J. Funct. Anal. 144 (1997), no. 2, 542-556. MR1432597(98e:46030)

[ARR] Aleman, Alexandru, Richter, Stefan, and Ross, William T., Pseudocontinuations and the backward shift. Indiana Univ. Math. J. 47 (1998), no. 1, 223-276. MR1631561 (2000i:47009)

[ARS1] Aleman, Alexandru, Richter, Stefan, and Sundberg, Carl, The majorization function and the index of invariant subspaces in the Bergman spaces. J. Anal. Math. 86 (2002), 139-182. MR1894480 (2003g:30058)

[ARS2] Aleman, Alexandru, Richter, Stefan, and Sundberg, Carl, Nontangential limits in $\mathcal{P}^{t}(\mu)$ spaces and the index of invariant subspaces, preprint.

[ARS3] Aleman, Alexandru, Richter, Stefan, and Sundberg, Carl, Invariant subspaces for the backward shift on Hilbert spaces of analytic functions with regular norm, Bergman spaces and related topics in complex analysis, Contemp. Math., 404, American Mathematical Society, Providence, RI, 2006. MR2244001

[ABFP] Apostol, C., Bercovici, H., Foiaş, C., and Pearcy, C., Invariant subspaces, dilation theory, and the structure of the predual of a dual algebra. I. J. Funct. Anal. 63 (1985), no. 3, 369-404. MR 0808268 (87i:47004a)

[Ar] Aronszajn, N., Theory of reproducing kernels, Trans. Amer. Math. Soc. 68, (1950), 337404. MR.0051437(14:479c)

[Be] Bercovici, Hari, Factorization theorems and the structure of operators on Hilbert space. Ann. of Math. (2) 128 (1988), no. 2, 399-413. MR0960951(89i:47032)

[BSZ] Brown, Leon, Shields, Allen, and Zeller, Karl, On absolutely convergent exponential sums. Trans. Amer. Math. Soc. 96 (1960) 162-183. MR0142763 (26:332)

[CEP] Chevreau, Bernard, Exner, George R., and Pearcy, Carl M., Boundary sets for a contraction. J. Operator Theory 34 (1995), no. 2, 347-380. MR1373328 (97b:47044)

[Co] Conway, John B., A course in functional analysis. Second edition. Graduate Texts in Mathematics, 96. Springer-Verlag, New York, 1990. xvi+399 pp. ISBN: 0-387-97245-5 MR.1070713(91e:46001) 
[Du] Duren, Peter L., Theory of $H^{p}$ spaces. Pure and Applied Mathematics, Vol. 38 Academic Press, New York-London, 1970, xii+258 pp. MR0268655 (42:3552)

[DS] Duren, Peter, and Schuster, Alexander, Bergman spaces. Mathematical Surveys and Monographs, 100. American Mathematical Society, Providence, RI, 2004. x+318 pp. ISBN: 0-8218-0810-9 MR2033762 (2005c:30053)

[Ga] Garnett, John B., Bounded analytic functions. Pure and Applied Mathematics, 96. Academic Press, Inc. [Harcourt Brace Jovanovich, Publishers], New York-London, 1981. xvi+467 pp. ISBN: 0-12-276150-2 MR0628971 (83g:30037)

[HKZ] Hedenmalm, Haakan, Korenblum, Boris, and Zhu, Kehe, Theory of Bergman spaces. Graduate Texts in Mathematics, 199. Springer-Verlag, New York, 2000. x+286 pp. ISBN: 0-387-98791-6 MR1758653 (2001c:46043)

[KT] Kriete, Thomas and Trent, Tavan, Growth near the boundary in $H^{2}(\mu)$ spaces. Proc. Amer. Math. Soc. 62 (1976), no. 1, 83-88 (1977). MR0454643(56:12892)

[MR] McCullough, Scott and Richter, Stefan, Bergman-type reproducing kernels, contractive divisors, and dilations. J. Funct. Anal. 190 (2002), no. 2, 447-480. MR1899491 (2003c:47043)

[NF] Sz.-Nagy, B. and Foiaş, C., Harmonic analysis of operators on Hilbert space. Translated from the French and revised, North-Holland Publishing Co., Amsterdam-London; American Elsevier Publishing Co., Inc., New York. MR0275190(43:947)

[Ni] Nikolski, Nikolai, Operators, Functions, and Systems: An Easy Reading, Volume 2: Model Operators and Systems, translated from the French by Andreas Hartmann and revised by the author. Mathematical Surveys and Monographs, 93. American Mathematical Society, Providence, RI, 2002. MR 1892647(2003i:47001b)

[Ri] Richter, Stefan, Invariant subspaces in Banach spaces of analytic functions. Trans. Amer. Math. Soc. 304 (1987), no. 2, 585-616. MR0911086 (88m:47056)

[RR] Rosenblum, Marvin and Rovnyak, James, Hardy classes and operator theory. Corrected reprint of the 1985 original. Dover Publications, Inc., Mineola, NY, 1997. xiv+161 pp. ISBN: 0-486-69536-0 MR1435287 (97j:47002)

[RS] Rubel, L.A. and Shields, A.L., The second duals of certain spaces of analytic functions. J. Australian Math. Soc., XI, part 3, (1970), 276-280. MR0276744(43:2484)

[Ru] Rudin, Walter, Real and Complex Analysis, 3rd edition, McGraw-Hill, New York, 1987. MR0924157 (88k:00002)

[Se] Seip, Kristian, Beurling type density theorems in the unit disk. Invent. Math. 113 (1993), no. 1, 21-39. MR1223222 (94g:30033)

[Sh] Shields, Allen L., Weighted shift operators and analytic function theory. Topics in operator theory, pp. 49-128. Math. Surveys, No. 13, Amer. Math. Soc., Providence, R.I., 1974. MR0361899 (50:14341)

[WY] Wu, Zhijian and Yang, Liming, The codimension-1 property in Bergman spaces over planar regions. Michigan Math. J. 45 (1998), no. 2, 369-373. MR.1637674 (99g:46025)

[Ya] Yang, Li Ming, Invariant subspaces of the Bergman space and some subnormal operators in $\mathbf{A}_{\mathbf{1}} \backslash \mathbf{A}_{\mathbf{2}}$. Michigan Math. J. 42 (1995), no. 2, 301-310. MR1342492 (96f:47013)

Department of Mathematics, Lund University, P.O. Box 118, S-221 00 Lund, Sweden

E-mail address: Aleman@maths.1th.se

Department of Mathematics, University of Tennessee, Knoxville, Tennessee 37996 1300

E-mail address: Richter@math.utk.edu

Department of Mathematics, University of Tennessee, Knoxville, Tennessee 37996 1300

E-mail address: Sundberg@math.utk.edu 